Cognitive Science

Faculty of Human Sciences

MACQUARIE UNIVERSITY

\title{
Predicting the actions of other agents
}

\author{
Lincoln John Colling
}

BSc, MSc

This thesis is presented for the degree of

Doctor of Philosophy

June 2012 



\section{Statement of candidate}

I certify that the work in this thesis entitled "Predicting the actions of other agents" has not previously been submitted for a degree nor has it been submitted as part of requirements for a degree to any other university or institution other than Macquarie University.

I also certify that the thesis is an original piece of research and it has been written by me. Any help and assistance that I have received in my research work and the preparation of the thesis itself have been appropriately acknowledged.

In addition, I certify that all information sources and literature used are indicated in the thesis.

The research presented in this thesis was approved by Macquarie University Ethics Review Committee, reference number: HE228NOV2008-DO6242L\&P

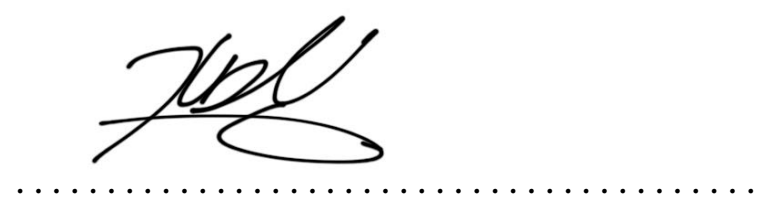

Lincoln John Colling

May, 2012 



\begin{abstract}
About this thesis
This thesis has been prepared in accordance with the Macquarie University Journal-articleformat thesis guidelines. Each chapter has been written in the format of a self-contained journal article. Where possible, all attempts have been made to minimize any referencing and stylistic inconsistencies between the chapters.

Some of the work presented in this thesis has been published elsewhere. A list of publications is provided below:

Chapter 2 has appeared as,

Colling, L. J., Thompson, W. F., and Sutton, J. (2010). Action synchronization with biological motion. In W. Christensen, E. Schier, and J. Sutton (Eds.), ASCS2009: Proceedings of the 9th Conference of the Australasian Society for Cognitive Science (pp. 49-56). Sydney: Macquarie Centre for Cognitive Science.

Chapter 6 has appeared as,

Colling, L. J. and Thompson, W. F. (in press). Music, Action and Affect. In T. Cochrane, B. Fantini, and K. R. Scherer (Eds.), The Emotional Power of Music. London: Oxford University Press

Appendix A has appeared as,

Colling, L. J. and Roberts, R. P. (2010). Cognitive psychology does not reduce to neuroscience. In W. Christensen, E. Schier, and J. Sutton (Eds.), ASCS2009: Proceedings of the 9th Conference of the Australasian Society for Cognitive Science (pp. 41-48). Sydney: Macquarie Centre for Cognitive Science.
\end{abstract}





\section{Acknowledgments}

First, and foremost, I would like to thank my supervisors Prof Bill Thompson and Prof John Sutton for their help throughout my candidature. I could not have asked for more knowledgeable, more supportive, or more understanding supervisors. I would also like to thank my associate supervisor Assoc Prof Mark Williams for all his advice and guidance during my candidature. In addition, I would also like to thank Prof Max Coltheart. Without his support, I would not be where I am today.

I am also grateful to the members of the various labs and groups that I have had the honour of being a part of during my time at Macquarie, including: the Music, Sound, and Performance Lab; the Perception in Action group; and the various research groups and reading groups that I've been involved with.

I would also like to thank Lesley McKnight, Lisa Yen, Katie Webb, Craig Richardson, and the various support and administrative staff who have made sure that I always filled in the correct forms when needed and had the equipment I needed to do my research.

Additionally, I would also like to thank Macquarie University and MACCS for their financial support in the form of a Macquarie University Research Excellence Scholarship, Postgraduate Research Fund, and various departmental grants.

Furthermore, I would like to thank the various groups and people that provided me with help or feedback on specific chapters, including: Rachel Bennetts and Bojan Neskovic for their help with data collection for Chapter 2 and Chapter 3; Kate Stevens for her feedback on Chapter 2 and Chapter 3 and for her comments that led to the experiments in Chapter 4; Michael Spivey for his comments that led to the experiments in Chapter 4; the students that generously agreed to part with their time to take part in the experiments reported in Chapter 2, 3, and 4; Glenn Carruthers for his comments on Chapter 5; Natalie Sebanz and two anonymous reviewers for their comments on Chapter 5; Günter Knoblich and the Sombies for their comments on Chapter 5; Michael Pauen and the Pauen colloquium for their comments on Chapter 5; Tom Cochrane for his comments on Chapter 6; Reece Roberts for his help in preparing Appendix A; and Jonathan Opie for his comments on Appendix A.

Last, but by no means least, I would also like to thank my partner, Bernice Plant, for her support over the past few years. Without her reminding me to eat when I was absorbed in my work, I would probably not have made it to the end. 



\section{Contents}

Thesis summary $\quad$ xv

I Introduction $\quad 1$

1 Introduction and background 3

1.1 Perceiving and acting . . . . . . . . . . . . . 5

1.2 Common-coding and action control . . . . . . . . . . . . . . 11

1.3 Evidence for the links between perception and action . . . . . . . . . . 13

1.4 Canonical neurons and mirror neurons . . . . . . . . . . . 16

1.5 Action prediction . . . . . . . . . . . . . . . . . 21

1.6 Aims of the current thesis . . . . . . . . . . . . . . . 30

1.7 References . . . . . . . . . . . . . . . . . 32

II An empirical investigation of action prediction 39

2 Action synchronisation with biological motion 41

2.1 Introduction . . . . . . . . . . . . . . . . . . . 41

2.2 Methods and materials . . . . . . . . . . . . . . . . . . . 47

2.3 Results . . . . . . . . . . . . . . . . . 52

2.4 Discussion ............................ 54

2.5 References .............................. 58

3 Motor experience modulates limb and joint information 61

3.1 Introduction . . . . . . . . . . . . . . . . 61

3.2 Methods . . . . . . . . . . . . . . . . . . . . . . 69

3.3 Results . . . . . . . . . . . . . . . . . . . . 74

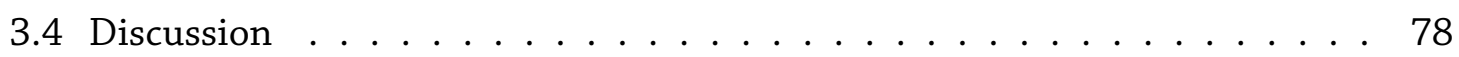

3.5 References .......................... 84

4 The effect of tempo and dynamic cues $\quad 87$

4.1 Introduction . . . . . . . . . . . . . . 87 
4.2 Experiment $1 \ldots \ldots \ldots \ldots \ldots$. . . . . . . . . . . . . . 93

4.3 Experiment $2 \mathrm{a}$ and $2 \mathrm{~b} \ldots \ldots \ldots \ldots$

4.4 General discussion . . . . . . . . . . . . . . . . . . . . . 105

4.5 Conclusions . . . . . . . . . . . . . . . . . . . . . . 107

4.6 References . . . . . . . . . . . . . . . . . . 107

III A theoretical framework for joint action 109

5 From action control to joint action 111

5.1 Introduction . . . . . . . . . . . . . . . . . . . . . . . 111

5.2 Predictive models in control theory . . . . . . . . . . . . . . . . . 118

5.3 Prediction and action control . . . . . . . . . . . . . . . . . . . 121

5.4 Common mechanisms for individual and joint action . . . . . . . . . . . 122

5.5 Phenomenology of joint action . . . . . . . . . . . . . . . . . . 140

5.6 Conclusions . . . . . . . . . . . . . . . . . . . . . . . . . . . 148

5.7 References . . . . . . . . . . . . . . . . . . . . 149

IV Action synchronisation in other contexts 157

6 Music, action, and affect $\quad 159$

6.1 Music and gesture . . . . . . . . . . . . . . . . . . 160

6.2 The influence of gesture on music perception . . . . . . . . . . . . 161

6.3 The influence of gesture on affective judgements . . . . . . . . . . . 165

6.4 Music and action . . . . . . . . . . . . . . . . . . . . 168

6.5 Action and emotion . . . . . . . . . . . . . . . . . . . 172

6.6 Expectancy, action, and emotion . . . . . . . . . . . . . 176

6.7 A feedback-controlled synchronisation model . . . . . . . . . . . 178

6.8 References . . . . . . . . . . . . . . . . . . . . . 183

$\begin{array}{lr}\text { V Conclusions } & 189\end{array}$

7 Conclusions 191

7.1 Part II: An empirical investigation of action prediction . . . . . . . . . . . 192

7.2 Part III: A theoretical framework for joint action . . . . . . . . . . . . . 196

7.3 Part IV: Action synchronisation in other contexts _ . . . . . . . . . 198

7.4 Implications and directions for future research . . . . . . . . . . . . . 199

7.5 References . . . . . . . . . . . . . . . . . . . . . 201 
$\begin{array}{ll}\text { VI Appendix } & 203\end{array}$

A Cognitive science does not reduce to neuroscience 205

A.1 Introduction . . . . . . . . . . . . . . . . . . 205

A.2 The role of neuroscience . . . . . . . . . . . . . . . 206

A.3 The role of brain imaging . . . . . . . . . . . . . . . . . . . . 213

A.4 An extended mind argument against reduction . . . . . . . . . . . 217

A.5 Conclusion . . . . . . . . . . . . . . . . . . 223

A.6 References . . . . . . . . . . . . . . . . . . . . . . 224 


\section{List of Figures}

1.1 An example of an emulator system. . . . . . . . . . . . . . . . . 10

2.1 The zigzag (left) and wave (right) patterns used as stimuli during the recording session. . . . . . . . . . . . . . . . . 46

2.2 Marker positions for the 11 reflective markers used during the recording session. . . . . . . . . . . . . . . . . . 48

2.3 Sample of the 3-D animated character used during the test session. . . . . . 50

2.4 Mean timing error for self-generated and other-generated actions. . . . . . 53

2.5 The relationship between the size of the authorship effect and tempo difference (left) and the size of the authorship effect and peak irregularity (right). 54

3.1 The zigzag (left) and wave (right) patterns used as stimuli during the recording session. . . . . . . . . . . . . . . 70

3.2 Marker positions for the 11 reflective markers used during the recording

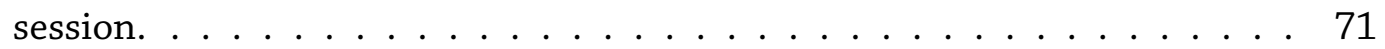

3.3 Example stimuli from the full information condition (left) and the point information condition (right). . . . . . . . . . . . 73

3.4 Absolute timing error for the full information condition and point information condition for the motor experience group and the naïve group. . . . . 74

3.5 (A) Evenly spaced button-presses results in timing errors that vary as a function of peak number. (B) Timing error that does not vary as a function of peak number is a result of the timing of button-presses varying as a function of peak number. . . . . . . . . . . . . . 76

3.6 Timing error as a function of peak position for the motor experience group and the naïve group. . . . . . . . . . . . . . . . 77

4.1 The zigzag (left) and wave (right) patterns used as stimuli during the recording session. . . . . . . . . . . . . . . . . . . 94

4.2 Marker positions for the 11 reflective markers used during the recording session. . . . . . . . . . . . . . . . . 95

4.3 Example stimuli from Experiment 1 (left) and Experiment 2a and Experiment $2 \mathrm{~b}$ (right). . . . . . . . . . . . . . . . . . 96 
4.4 Absolute timing error for natural velocity stimuli and constant velocity stimuli for each of the four experimental blocks (Experiment 1). . . . . . . . . 98

4.5 Absolute timing error for natural velocity stimuli and constant velocity stimuli for each of the four experimental blocks (Experiment 2a) . . . . . . . 103

4.6 Absolute timing error for natural velocity stimuli and constant velocity stimuli for each of the four experimental blocks (Experiment 2b). . . . . . . . 104

6.1 An illustration of the synchronisation feedback model (reprinted by permission from Thompson \& Quinto, in press) . . . . . . . . . . . . . . . 181 

Thesis summary

The ability to predict the actions of other agents is vital for joint action tasks where the actions of two actors must be coordinated in a timely fashion. Recent accounts suggest that action prediction might be implemented by a system that allows actors to generate predictions about perceived actions by emulating those actions using their own motor systems. In particular, it has been suggested that actors are able to use their own inverse models for action to predict what actions another agent might select in order to realise a particular goal, and that actors are able to use their own forward models for action to generate predictions about the unfolding dynamics of perceived actions. The body of this thesis is divided into three sections (Part II-IV) and examines the empirical support for these hypotheses as well as their theoretical implications. In Part II, Chapter 2 confirms that observers are able to generate more accurate predictions when their own movement dynamics more closely match the movement dynamics of the observed action. Furthermore, the results also suggest that the output of the prediction system is available in a timely fashion suggesting that it can be used for joint action coordination. Chapter 3 examines what factors might assist observers to reconstruct the motor codes responsible for generating perceived actions by specifically examining the influence of limb and joint information on the prediction of perceived actions. The results suggest that limb and joint information allows observers to generate more accurate predictions about observed actions, but that this effect is limited to those observers who have motor experience with the 
observed actions. Chapter 4 rules out an alternative explanation for the results obtained in Chapter 2, that differences in action prediction can be explained by self-similarity in action production between the action production and subsequent action prediction phases of the experiment. In Part III, Chapter 5 presents a framework for understanding joint action coordination wherein an attempt is made to extend well understood mechanisms of action control into the domain of joint action. This framework might assist with trying to understand the phenomenology of joint action and experiences such as group flow. In Part IV, Chapter 6 examines the role of action prediction mechanisms in a domain other than traditional conceptions of joint action. Specifically, it examines the role of action prediction and action synchronisation in musically evoked emotion. Finally, the implications of this thesis are discussed as they relate to our understanding of the nature of joint action, phenomenology of joint action, functions of the mirror-neuron system, and the role of sensorimotor systems in cognition. 


\section{Part I.}

\section{Introduction}



CHAPTER 1

\section{Introduction and background}

We live in a complex world full of objects and obstacles, and our ability to successfully navigate through this world is critical for our survival. The world, however, is not just a world of things, of objects that we might interact with, it is a world filled with creatures like ourselves, and it is a social world. If we are to flourish in a social world, we must have a means by which we can act together with others to achieve goals that we are unable to achieve acting alone. At first glance, the problem of navigating through a complex world and the problem of interacting with others might seem very dissimilar. The former is achieved through the interplay of our perceptual systems, which give us information about the world, and our action systems, which allow us to act in it. The latter might seem to only be the purview of those disciplines that study social relations, such as social psychology or sociology, and it appears to be a matter of understanding the minds of others. However, at its core, a social interaction or cooperative act is an action. How we select what action to perform is, in part, based on information received through our perceptual systems. Thus, our successful being in the world with others is as much a case of the interplay between perception and action as our being in the world of things. It is 


\section{Introduction and background}

this claim that lies at the heart of this thesis.

This thesis will argue that in order to develop a full understanding of how it is that we are able to act together with others we must understand the role played by our perception and action systems. This thesis takes as its target those interactions where two agents are able to tightly coordinate their actions; interactions where perception and action must work closely together so that co-actors are quickly able to select when to act and how to act in response to each other. This is not simply a case of observing and then acting; rather, these types of interactions may require co-actors to produce complementary actions that occur at the same time. To do this, an agent must be able to predict how and when their co-actor will act before they have acted. That is, actors must be able to predict the actions of their co-actors.

The primary aim of this thesis is to examine the mechanisms that underlie humans' ability to predict the actions of other agents. It will argue that in order to understand how this is possible we must understand the links between perceiving and acting. To do this, we cannot view perception and action merely as distinct and non-cognitive input and output systems. Rather, we must view perception and action as intimately linked, and we must understand how perception shapes action and, in particular, how action shapes perception. In addition, it will argue that this approach can also be extended to understand other social phenomena such as music and, particularly, emotional expression in music.

The body of the thesis is divided into three parts (Part II-IV). Part II deals with the mechanisms that underlie action prediction. Specifically, I argue that action prediction is supported by mechanisms that allow observers to generate an internal model of the observed action, a model that is isomorphic with the observed agent (Chapter 2). I will also argue that this internal model is influenced by motor experience with the observed action (Chapter 3 ). Chapter 4 rules out an alternative explanation for the data presented in Chapter 2, that the authorship effect arises due to a coincidence of preferred tempo for producing rhythmic movements in the action production and action synchronisation phases of the experiments. In Part III (Chapter 5), I present a framework that incorporates 
the mechanisms responsible for action prediction into the larger context of joint action. I argue that similar planning and predictive mechanisms underlie both action prediction in the intrapersonal context and action prediction in the interpersonal context. Finally, in Part IV (Chapter 6), I examine a different social activity, namely music. I will argue that music should not be understood as a purely auditory phenomenon. Rather, music is a multi-modal phenomenon that critically relies on the tight links between perception and action. And, furthermore, I will argue that in order to understand how emotion is expressed and communicated by music, we must understand how music engages with these perception-action links.

\subsection{Perceiving and acting}

In the section that follows, I first outline the traditional view of perception and action, which views perception and action as not only wholly distinct systems but also separate from cognition. Following this, I will outline an approach that attempts to unify perception and action and views perceptual and action systems as a proper part of cognition.

\subsubsection{The traditional approach to perception and action}

According to the approach to visual perception typified by researchers such as David Marr (1982), visual perception is a process primarily concerned with recovering a representation of the world "out there" from the array of intensity values detected by the retina. Marr (1982) describes a process of computing ever more elaborate representations leading eventually to the construction of a three-dimensional model of the scene in view. Marr (1982) wanted to develop an account of how it is that people can see unfamiliar objects and, therefore, had to make do without top down processing influencing the construction of this representation; although, he did allow for top down processing to influence object categorisation (Boden, 2006). Thus, his account excluded any role for action in modulating perceptual processing. To be sure, Marr (1982) suggested that vision could be used 


\section{Introduction and background}

for a number of different purposes, including directing action; however, action played no part in his account of perception. Perception was an input, but action was only an output.

The view that action functions merely as an output, the end product of a long chain starting with perception, is also found in a number of other accounts. It can be traced back at least as far as Descartes (1606/1998), who developed the concept of the reflex arc. Descartes (1606/1998), in describing how the body might be moved in response to a stimulus, such as fire felt near the foot, describes how the force exerted by the fire might be transmitted upward toward the brain. Once this force reaches the brain, pores on the internal surface of the brain are opened and animal spirits are carried from the cavity in the brain back to the foot to cause the muscle to move the foot to safety. The Cartesian view of action is also implicit in the approach of Donders (1869/1969). Donders (1869/1969) outlines a method by which it might be possible to measure the speed of mental processes in a manner similar to how Helmholtz had already measured the speed of nerve impulses. To do so, Donders (1869/1969) divided the process of generating a response to a stimulus into a number of stages including the action of the sensory nerves at the start and action of the motor nerves at the end, much as Descartes had done. Also included was the activity of "the organ of conception", which we might consider to be the process of stimulus identification, and the activity of "the organ of the will", which we might consider to be the process of response selection. The view of Donders (1869/1969) was to influence Sternberg (1969), and was to lead to the linear stage model that held the view that a task such as responding to a stimulus required at least two stages of processing-a stimulus encoding stage and a translation and response organisation stage-that were connected in a linear, unidirectional manner. Stimuli were to be coded in terms of patterns of stimulation received from the sensory organs and responses were to be coded in terms of patterns of muscle activation, with translation required to transform one set of representations into the other (Prinz, 1997b). Thus, as noted by Prinz (1997b), the linear stage model was to stress the incommensurability of perception and action.

The approach of Sternberg (1969) was also to fit well with the view of cognition ad- 
vocated by Newell and Simon (e.g., Newell, 1994; Newell \& Simon, 1961, 1997). Newell (1994), in describing the process of making a speeded response to a stimulus, divides perception, central cognition, and action into three distinct parts connected in a linear, unidirectional manner. In this scheme, perception stages encode a stimulus from the environment into a representation that could be operated on by central cognition. After the relevant processing, a command could be decoded and passed on to produce motor output. Perception and action were not seen to be under the control of central cognition, and central cognition was seen to operate by transforming symbolic representations according to syntactic rules (see Newell, 1994; Newell \& Simon, 1997). In this way, not only were perception and action seen as distinct input and output systems, but they were also wholly separated from the real business of cognition.

\subsubsection{Rejection of the traditional view}

While the views that see perception and action as wholly distinct have been very influential, alternative views have stressed the links between perception and action. One such alternative was put forward by Gibson (1986). Where Marr (1982) had argued that the purpose of perception was to recover the three-dimensional structure of the world from the two-dimensional pattern on the retina, Gibson instead argued that visual perception was the process of "seeking and extracting information about the environment" (Gibson, 1966, p. 5), and he argued that perception involved the "exploratory activity of looking around, getting around, and looking at things." (Gibson, 1986, p. 147). Note that for Gibson (1986) the environment is something different to the physical world. An environment is something that is specific to an organism - it is what an organism perceives - and part of the environment that an organism perceives are its affordances. Affordances are opportunities for action that the environment offers an animal. For example, a surface that is horizontal, sufficiently flat and large, and made of a substance that is sufficiently rigid might afford support. This affordance is measured relative to an animal-a surface that might afford one animal support might not, due to a difference in weight or size, af- 


\section{Introduction and background}

ford support for another. Although affordances are measured relative to the animal they are not determined by the animal's current needs. That is, an apple might afford biting whether the animal perceiving it is hungry or not. Affordances are always out there in the environment ready for the animal to perceive them. In this way, action is built right into Gibson's (1986) view of perception, with the action capabilities of the animal determining how it perceives the world. For Gibson, affordances were not only properties of objects in the environment, but also properties of other organisms. Indeed, Gibson (1986, p. 135) wrote that "[t]he richest and most elaborate affordances of the environment are provided by... other people." While Gibson's view was radical, and has not been met with widespread acceptance, it does provide an important insight. That is, that perceptual processing of the actions of other people might not only be a function of the observed actions, but might also be a function of the observer's action system. This insight guides much of the work presented in this thesis.

Gibson's (1986) view has also had an influence that has extended beyond just understanding perception and action. It has also been influential in shaping a view of cognition that departs from the orthodoxy as exemplified by, for example, Newell and Simon (e.g., Newell, 1994; Newell \& Simon, 1961, 1997). This view, broadly termed embodied cognition, has not only rejected the distinction between perception and action, but has also sought to reunite perception and action with cognition. For example, Varela, Thompson, and Rosch (1991) put forward a view of cognition as embodied action, which views "sensory and motor processes, perception and action, [as] fundamentally inseparable in lived cognition." (p. 173). Clark (1997) too has aimed to put action, and the body, back into cognition. By modifying Gibson's (1986) notion of affordances, Clark (1997) argued that, rather than viewing cognition as a process that operated on symbolic, action-neutral representations (as argued by, for example, Newell \& Simon, 1997), representations should be viewed as action-centred.

Wilson (2002) identifies six broad claims made by the embodied cognition movement. First, cognition is situated-it is not detached from the environment in which it takes 
place. Second, cognition is time pressured. Third, some of the work of the cognitive system is offloaded onto the environment. Fourth, the environment is part of the cognitive system. ${ }^{1}$ Fifth, cognition is for action-that is, the primary business of cognition is for getting around in the world. The last claim is that off-line cognition is grounded in the body. That is, rather than cognition operating on symbols, it employs structures that originally evolved for sensorimotor control, which are run off-line decoupled from the environment. These final two claims, and the last claim in particular, will be important to the argument developed in this thesis.

The concept of emulators, developed by Grush (1997, 2004), provides an example of how sensorimotor systems can be used in off-line cognition. Emulators, a concept borrowed from control theory (for an introduction to control theory, see Golnaraghi \& Kuo, 2010), are devices that model the input-output relations of a system. To explain what this means a simple example from Grush (1997) will be useful. Grush (1997) describes a simple system composed of a controller that issues commands to a target system-a room fitted with a heating and cooling system, which the controller is able to control, and temperature sensors that are able to send feedback back to the controller. The controller is able to control the temperature in the room by issuing commands to the heating and cooling systems. To set the temperature to a desired goal state, the controller can read the temperature from the sensor and adjust the heating and cooling as appropriate. Such a setup works well when the feedback from the sensor can arrive in a timely fashion. However, when feedback delays are introduced—for example, by placing the room on a space station while the controller remains on Earth-the controller will no longer be able to control the temperature of the room because the information that the controller acts on will be out of date and, as a result, the control commands issued by the controller may be inappropriate for the current state in the room. The problem of feedback delays can be overcome by using an emulator, or forward model, that models the forward dynamics, or input-output relations, of the target system. With an emulator in place, the controller

\footnotetext{
${ }^{1}$ See the Appendix for a discussion of the implications of points two through four.
} 


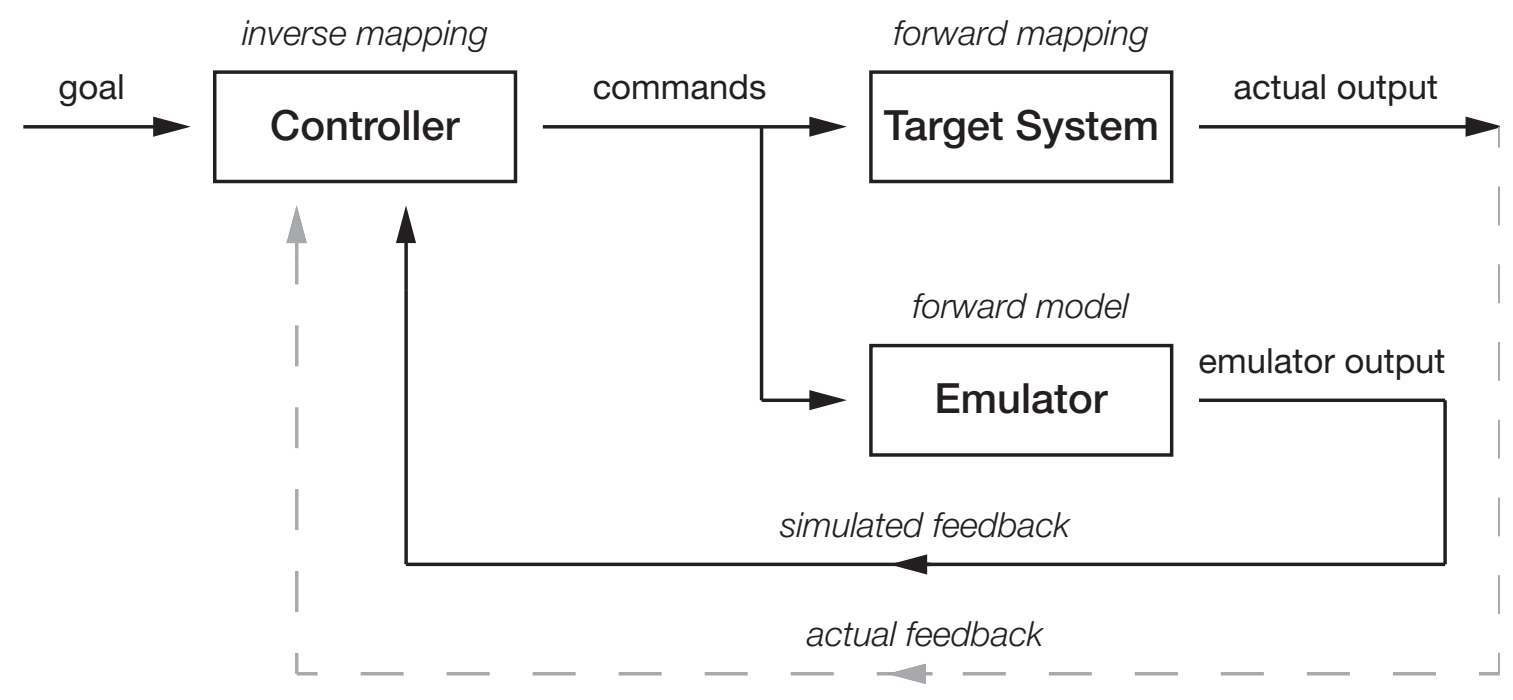

Figure 1.1. An example of an emulator system.

can send one copy of the control commands to the target system and another copy to the emulator, which then behaves as the target system would. Feedback from the emulator can then be substituted for feedback from the target system thereby making it possible to bypass the transmission delays. This is illustrated in Figure 1.1.

Importantly, emulators can also be used for reasoning and planning. Grush (1997) cites the example of a robot that has the task of moving its arm around while avoiding obstacles. In addition to the arm, the robot is fitted with a video camera that can monitor the movements of the arm. This system can then be equipped with an emulator that learns the relationship between specific commands sent to the arm and specific visual input received from the camera. Once the emulator has learnt this mapping, the arm and camera can be taken off-line and the robot can plan an action by sending commands to the emulator (instead of the arm) and reading visual feedback from the emulator (instead of the camera). If the robot is given the task to move its arm to a specific location while avoiding obstacles at other locations, the robot can use the emulator to try out different commands until it is able to determine which one is appropriate to achieve its goal. By doing so, the robot is able to plan and problem solve, but this planning then becomes a function of the robot's perception and action systems. In Part II and Part III, I will argue that emulators are also useful for understanding how it is possible to coordinate actions with other people. 


\subsection{Common-coding and action control}

Two recent approaches, common-coding theory-and its more recent formulation, the theory of event coding (Hommel, Müsseler, Aschersleben, \& Prinz, 2001)—and the model of action control developed by Wolpert and colleagues (Wolpert, 1997; Wolpert \& Kawato, 1998) have also argued for a tight coupling between perception and action. Each of these approaches predicts bidirectional influences between perception and action, and insights gained from these frameworks are important for understanding the work presented in the current thesis.

According to the theory of common-coding, rather than perception and action codes being incommensurate, as suggested by the linear stage model (see Section 1.1.1), perception and action are seen as being coded within a single representational domain (Prinz, 1997a). That is, rather than being distinct, action codes and perception codes are merely seen as different ways of doing the same thing-representing external events (Hommel et al., 2001). This approach shares with Gibson (1986) the notion that perception is not passive, but is an active process of acquiring information about the environment through active movement of the head, eyes, and body. Producing action leads to perception. This can be seen in the simple case of moving one's hand. When one's hand moves it is not only an action, but the movement of the hand is also perceived. Thus, action and perception are merely different ways of representing that single event.

The link goes both ways-perception is also linked to action planning. Actions are planned according to their goals, and goals are events in the external world. To move one's hand in such and such a manner is to bring about a particular perceptual event. Thus, action planning is linked to perceptual prediction because to plan an action is to predict that perceptual event. To illustrate, Hommel (2009) describes a process by which a baby through, for example, motor babbling might produce movements that, in turn, produce certain perceivable consequences. Through Hebb's law, these motor activations get associated with their perceivable effects. This forms an action-effect link that represents both the action and the effect. With these action-effect links in place, it is then possible to plan 


\section{Introduction and background}

actions on the basis of their effects. Activating the code for a particular perceivable effect will, in turn, bring about the action that produces that effect.

Wolpert and colleagues (Wolpert, 1997; Wolpert \& Kawato, 1998) have suggested a similar idea, but framed in terms of forward and inverse models. Forward models, which are equivalent to Grush's (1997) emulators, have already been introduced above; inverse models are the other major component in a control system, and they are used as system controllers. They provide a means by which to compute the control commands that would be required to produce a particular behaviour. For example, in a goal-directed system such as an arm, an inverse model can be used to compute the motor commands needed to move the arm in a particular fashion. Forward models, on the other hand, are used to predict the effects of the motor command selected by the inverse model. Wolpert and colleagues (Wolpert, 1997; Wolpert \& Kawato, 1998) suggest that two types of forward models are used in action control. The forward dynamic model predicts what the next state of the limb will be, given a particular motor command and a particular current state, while the forward output model predicts the sensory feedback that will occur, given the prediction generated by the dynamic model. The predictions generated by the forward model can be used for a number of different purposes. These include: controlling movement in the absence of feedback by substituting the predictions of the forward model for actual feedback, and isolating the sensory effects of movement from those that arise in the environment by subtracting the predicted sensory feedback from the actual sensory feedback.

Because forward models can be used to predict the sensory consequences of an action, Wolpert and colleagues (Wolpert, 1997; Wolpert \& Kawato, 1998) suggest motor learning and action planning occur in a manner similar to that suggested by the theory of event coding (Hommel, 2009). During motor learning, motor commands can be generated that produce particular outputs. These outputs can be compared to the predictions generated by the forward model, and the discrepancy between the actual output and predicted output can be used as a training signal. In this way, particular motor commands can become associated with particular sensory consequences. Once this association is formed, actions 
can then be selected on the basis of their sensory effects. This is similar to Grush's (1997) robot example where the robot can select the correct control command by generating a prediction of how a particular control command will change what it perceives through its camera.

\subsection{Evidence for the links between perception and action}

Over recent decades, the empirical data has increasingly been found to support views that argue for a unification of perception and action and a role for sensorimotor systems in cognition. Indeed, the literature abounds with examples of the connections between perception and action, with perception influencing action and action influencing perception. The section that follows outlines a few examples from this literature.

One of the most well-known examples of the influence of perception on action planning comes from studies employing stimulus response compatibility (SRC) paradigms, with the most well-known of these being the Simon task. While a complete review of the SRC literature is not possible it is worth at least introducing the concept. The general format of the Simon task is that subjects are required to respond to one dimension of a stimulus while ignoring an irrelevant spatial dimension of the stimulus. The general finding is that the irrelevant spatial dimension facilitates responses that are spatially compatible with it. For example, in the early experiments by Simon (e.g., Simon, 1969), subjects were asked to move a control handle either to the right or to the left in response to a tone played in either the right or the left ear. Depending on the experimental block, subjects were asked to either turn the handle away from the ear of stimulation or towards the ear of stimulation. The results showed that subjects were faster when they were required to turn the handle towards the ear of stimulation, suggesting that the irrelevant spatial dimension of the stimulus (the ear of stimulation) facilitated responses that were spatially compatible. The task can also work in the visual domain. For example, Hedge and Marsh (1975) presented subjects with red and green lights that were displayed either in the left or right of a subject's field of view. Subjects were required to respond to the colour of 


\section{Introduction and background}

the stimulus using buttons located on the left and right while ignoring the location of the light. The results showed that when the stimulus was located on the same side as the to-be-pressed button, responses were faster relative to the case where the stimulus was located on the opposite side of the to-be-pressed button.

While experiments employing SRC tasks illustrate how perception can influence action planning, these effects are not unidirectional. A variant of the SRC task has also shown that action planning can influence perception. This reversal of SRC, termed actioneffect blindness, has been demonstrated by Müsseler and Hommel (1997) using a stimulus masking paradigm. In these experiments, subjects were first shown a masked stimulus (a left or right pointing arrow) before executing a pre-planned action of either a left hand or right hand button press. After the response, they were asked to report the identity of the masked stimulus - whether it was a left or right pointing arrow. The results showed that subjects were less likely to correctly identify the masked stimulus when it was compatible with the pre-planned action. That is, the nature of the action plan that subjects formed influenced their perception of the stimulus. This decreased sensitivity may result because the action plan activates a common representation for the action and the compatible stimulus and this representation then enters a refractory period during which it is more difficult to reactivate that representation. As a consequence, the stimulus may not be sufficient to activate the representation, resulting in decreased sensitivity to the stimulus.

Experience of particular sensory-motor associations has also been shown to influence perceptual processing during concurrent action. For example, Repp and Knoblich (2007) asked trained pianists to play tone pairs by pressing keys on a keyboard (both a piano keyboard and computer keyboard). The tone pairs were perceptually bistable and could be heard as either a pair of ascending or a pair of descending tones. Keys on a piano are arranged with high pitches on the right and low pitches on the left, and when pianists make left-to-right key presses on a piano it is always accompanied by ascending tones. Similarly, right-to-left key presses are always accompanied by descending tones. Repp and 
Knoblich (2007) predicted that if the action used to produce the ambiguous tone affected the perception of the tones, then pairs produced with left-to-right key presses would be more likely to be judged as ascending compared with pairs produced with right-to-left key presses. The results confirmed this prediction. Importantly, the effect was not replicated in a control group of non-pianists, which suggests that the action-effect associations that pianists learn during their training are capable of influencing perception during concurrent action.

From the evidence cited above it is clear that concurrent action or action planning can influence perception. However, what is most interesting for the purposes of this thesis is that not only can concurrent action influence perception but the action possibilities of the perceiver, or the manner in which a perceiver produces actions, can also influence perception. One such example relates to the two-thirds power law. The two-thirds power law is a law that constrains action production. It states that the speed at which a curved movement is produced is inversely related to the curvature of the movement trajectory (Viviani \& Terzuolo, 1982). In addition to constraining action production, this law has also been found to influence perception. Viviani and Stucchi (1992) asked subjects to view dynamic traces that traced out the shape of an ellipse, and to adjust the velocity profile of the traces so that the traces appeared to have a constant velocity. The results showed that subjects were biased towards selecting velocity profiles that were not constant but instead conformed to the two-thirds power law. That is, subjects selected the velocity profiles that would have been produced if they had themselves attempted to draw the ellipses with a constant velocity profile. This suggests that the constraints of the motor system also influence perceptual processing.

The action possibilities of an observer have also been shown to influence how they perceive movement of human bodies. For example, Shiffrar and Freyd (1990) report that when human bodies are used as stimuli in apparent motion displays the usual pattern of data is not replicated. In classic demonstrations of apparent motion, when two spatially separated stimuli are displayed sequentially (within a particular temporal window), sub- 


\section{Introduction and background}

jects report a percept of a single stimulus moving along the shortest path between the two spatial locations. However, when the stimuli used are human bodies a different pattern of data emerges. Rather than motion always appearing to occur along the shortest path, subjects could be biased toward perceiving the movement as travelling along a longer path when that longer path conforms to the anatomical constraints of the human body. Thus, it appears that the constraints of human action, or the action capabilities of the human body, influence perception.

\subsection{Canonical neurons and mirror neurons}

This tight link between perception and action has also been supported by recent discoveries in neuroscience. Rizzolatti et al. (1988) report a class of neurons, termed canonical neurons, located in area F5 of the macaque monkey premotor cortex that are active when the monkey performs specific motor acts, such as grasping food with its hand. Some neurons were not only activated by a particular motor act, but also showed selectivity for the way in which an act was performed. For example, some neurons might show increased activity with any instances of grasping-with-the-hand while others might be selective for grasping-with-the-hand using a precision grip or for grasping-with-the-hand using finger prehension. However, the most interesting feature of these neurons was that in addition to motor properties these neurons had visual properties. In particular, Rizzolatti et al. (1988) report a subset of grasping-with-the-hand neurons that were not only active when the monkey grasped with a precision grip but also when the monkey viewed a small object that might be grasped with a precision grip. That is, through the activity of these neurons, a neural representation of the action that might be used to interact with an object is activated by the passive observation of that object. Thus at a neural level there is a common representation for perception and action. Garbarini and Adenzato (2004) have argued that these neurons provide a neurophysiological basis for Gibson's affordances. Indeed, Gibson's description of affordances provides a good description of the properties of canonical neurons: "If the object is hand-size, it is graspable; if too large or too small, it 
is not. Children learn to see sizes in terms of prehension; they see the span of their grasp and the diameter of the ball at the same time." Gibson (1986, p. 234).

In addition to canonical neurons, area F5 also contains a different class of neurons called mirror neurons (di Pellegrino, Fadiga, Fogassi, Gallese, \& Rizzolatti, 1992). Unlike canonical neurons, which are active during an action and during observation of an object that affords that action, mirror neurons are active during both the production of an action and the passive observation of the same (or similar) actions. Thus they share the motor properties of canonical neurons but exhibit radically different visual properties. Although the properties of mirror neurons are now beginning to be well understood what is less clear is their psychological function. Several different accounts have been given for the psychological function of mirror neurons, including the action understanding account and the action prediction account.

\subsubsection{The action understanding account of mirror neuron function}

The action understanding account has been put forward in various forms, initially by the neuroscientists responsible for the discovery of mirror neurons (e.g., Gallese \& Goldman, 1998; Rizzolatti, Fogassi, \& Gallese, 2001). While it is outside of the scope of this introduction to review all the recent formulations of the action understanding account of mirror neuron function, the account of Rizzolatti et al. (2001) will suffice to show the general reasoning behind the approach. According to their account, one is able to understand observed actions because observing an action activates the motor representation responsible for that action. Importantly, these motor representations are sensitive to action goals-different populations will be active during reaching-to-grasp a cup with the intention to drink from it and reaching-to-grasp a cup with the intention to clean it (e.g., Iacoboni et al., 2005). Therefore, the motor activations caused by the mirror-neuron system provide a means by which to understand actions and infer action goals.

While various formulations of this basic account have been put forward they commonly share the idea that an action goal, attributed to the observed other, is the output of some 


\section{Introduction and background}

system involving mirror neurons. However, there is an alternative explanation for their function. Rather than the goals being produced as an output of the mirror-neuron system, goals might serve as the input with predicted actions being the resulting output.

\subsubsection{The action prediction account of mirror neuron function}

Two predictive accounts of mirror neuron function have been put forward by Wilson and Knoblich (2005) and Csibra (2008). ${ }^{2}$ These are not competing accounts, but rather are complementary, each dealing with different parts of the prediction process. Each of the accounts will be outlined individually, and then a suggestion of how they might complement each other will be made.

\subsubsection{Mirror neurons as inverse models}

According to Csibra (2008), mirror neurons form part of system that is used for predicting what motor commands might be employed to realise a particular goal. Thus, rather than goals being produced as the output of the mirror-neuron system, according to Csibra's (2008) account, they serve as the input. Csibra (2008) argues that much of the data that has been used to argue for the action understanding account of mirror neuron function actually supports this alternative account.

Data from Iacoboni et al. (2005) and Umiltà et al. (2001) have been used to argue that mirror neurons are sensitive to action goals. However, rather than their activity representing the goal, and being used to infer the goal, Csibra (2008) argues that these data show that mirror neuron activity is dependent on already knowing the goals of the other agent. For example, Umiltà et al. (2001) had macaque monkeys view a reach-to-grasp movement, while manipulating whether the action was directed toward a food object and whether the final part of the movement was visible or occluded behind a screen. The data showed that the mirror neurons were selective for the reach-to-grasp action in the presence of a food object but not for an identical action in the absence of a food object. The

\footnotetext{
${ }^{2}$ Work by Jacob (2008) provides a critique of the action understanding account of mirror neuron function and also advocates for a predictive account similar to Csibra (2008).
} 
authors argue that this result shows that mirror neurons are selective for goal-directed actions. The critical comparison, however, occurred when the action was occluded behind a screen. In the occluded viewing conditions, the monkey was first given information about whether or not a food object was placed behind an occluding screen. The monkey then viewed the action in a food-present and a food-absent condition. Again, the activity from the recorded mirror neurons was selective for the action performed in the food-present condition even though the object and the object-hand contact were not visible. The authors interpreted this result as showing that the activity of mirror neurons does not code for the action per se (as they are active even when the action is hidden from view) but instead signals that the given action has been recognised, or understood, as an instance of a particular goal-directed action.

Similarly, Iacoboni et al. (2005) have argued that the human mirror-neuron system also shows selectivity for action goals. In their study, observers viewed an action performed in two contexts while brain activity was recorded with fMRI. In both contexts, observers viewed a reaching action directed towards a cup, but with the action context determining the intention behind the action. In the "drinking tea" context, observers viewed a reaching action directed towards a cup that was performed with the intention of picking up the cup to drink from it. In the "washing up" context, the observers viewed a reaching action directed towards a cup that was performed with the intention of picking up the cup to take it away and clean it. In each case, additional objects were shown with the action to provide the context and so that the plausible intention could be inferred. In addition to the actionin-context displays, observers were shown the action alone and the context alone so that it was possible to isolate the brain activity related to the inferred intention. The results showed that certain regions within the mirror-neuron system were indeed sensitive to the inferred intention, and the authors argue that this implicates the mirror-neuron system in the process of understanding the intentions of others.

Csibra (2008), however, argues that instead of showing that the mirror-neuron system is involved in inferring intentions, these results show that the mirror-neuron system is 


\section{Introduction and background}

involved in determining the action that might be used to realise a particular goal. Indeed, the studies of Umiltà et al. (2001) and Iacoboni et al. (2005) demonstrate that the mirrorneuron system is selectively active when the context allows the observer to infer the action goal. Csibra (2008) argues that the mirror-neuron system acts as an inverse model (see Section 1.2), and that action goals are fed into this system with motor activity being the output. The mirror-neuron system can, therefore, form part of a system that is used to predict the actions of others. When their goals are understood, the mirror-neuron system can be used to predict what actions they might perform to realise those goals.

\subsubsection{Mirror neurons as forward models}

While Csibra's (2008) account suggests that the mirror-neuron system functions as an inverse model, Wilson and Knoblich's (2005) account suggests that the mirror-neuron system functions as a forward model. According to this account, observers use the mirrorneuron system as an emulator for the actions of the observed agent. The output of the emulator, which runs in parallel with the observed actions, is available more rapidly than information from the external world and, therefore, this output can be used to stand in for information from the external world (see also the space station example from Grush, 1997, outlined in Section 1.1.2). The forward model is instantiated within the observer's own motor system and, therefore, the dynamics of their forward model should conform to the dynamics of the observer's motor system. This allows an observer to predict the unfolding dynamics, and the sensory consequences, of an observed action using knowledge embodied in their own motor system.

\subsubsection{The emulator hypothesis of action prediction}

Rather than being mutually exclusive, the accounts of Wilson and Knoblich (2005) and Csibra (2008) are complementary. Both accounts suggest that action prediction relies on the observer's motor system. However, Csibra's (2008) account is focussed on the problem of predicting the motor codes that might be employed by an observed other, while Wilson 
and Knoblich (2005) provide an account of how the unfolding dynamics of an action are predicted. Both of these functions are important, and an action prediction system that is capable of supporting joint action will not only need to predict what actions a conspecific will select to realise a particular goal, but also more fine-grained details about how they will execute the actions - such as details about their timing or predictions about the sensory effects the action will produce. By combining these accounts, it will be argued that the observer's entire action system forms part of an emulator system. That is, it is a system that replicates the properties of one system-the observed motor system-using different means-the observer's motor system. I will refer to this as the emulator hypothesis of action prediction.

Furthermore, while both Csibra (2008) and Wilson and Knoblich (2005) attempt to provide an account of the psychological function of the mirror-neuron system, the position defended in this thesis is not explicitly based on the mirror-neuron system. Ultimately, I believe an account of the emulator system must be based on behavioural evidence. Properties of the mirror-neuron system may prove useful for generating hypotheses and conjectures about the properties of the emulator system, but as these ultimately must be tested against behavioural data the theory need not be tied to the mirror-neuron system. This is the approach adopted in thesis. ${ }^{3}$ A key prediction of the emulator hypothesis is that the observer's motor system should influence any predictions that they generate. As the argument will ultimately turn on behavioural evidence this will be the focus of the section that follows.

\subsection{Action prediction}

One way to examine the role of the motor system in action prediction is to employ paradigms that look for motor traces in the predictions that subjects generate. This can be done by, for example, comparing the relative prediction capabilities of subjects with par-

\footnotetext{
${ }^{3}$ See the Appendix for an extended discussion of the relationship between neuroscience and psychology.
} 


\section{Introduction and background}

ticular differences in their motor systems, such as motor expertise, or by examining action prediction as a function of a subject's kinematics. The section that follows outlines some of the literature on action prediction detailing first the differences between experts and novices and then introducing the self/other paradigm that has been used to examine prediction as a function of a subject's kinematics. Following this, I outline the literature that has employed a synchronisation paradigm to examine how rapidly the prediction system operates.

\subsubsection{Prediction}

Neurophysiological evidence from dancers has shown that, relative to novices, the mirrorneuron system of experts is activated more strongly during observation of actions that are part of their own action repertoire (Calvo-Merino, Glaser, Grézes, Passingham, \& Haggard, 2005). If the mirror-neuron system is involved in action prediction, then we might also expect to find differences in action prediction as a result of differences in motor expertise. Several early studies adopted the approach of comparing action prediction for non-experts and relative experts. For example, Isaacs and Finch (1983) compared beginning and intermediate tennis players on their ability to predict the landing position of a serve. The results showed that intermediate players were generally more accurate at picking the landing position than those players who were just starting out. Furthermore, prediction was more accurate with increased viewing times. Similar results have been reported by Abernethy (1990). In this study, novice and expert squash players were tested on their ability to predict the direction and force of a stroke made by another player. They employed a temporal occlusion paradigm where the entire movement was divided into several sequences including the movement before racquet-ball contact as well as after racquet-ball contact. The results showed that experts were better under all levels of temporal occlusion including pre-contact sequences. When comparing specific temporal windows, the results showed that experts were able to exploit cues present in the pre-contact sequences whereas novices were only able to exploit the cues present in post-contact se- 
quences. This suggests that novices might rely more on cues such as ball movement while experts may be able to generate their predictions based on the movement of the player. Interestingly, the accuracy scores of the expert players were highly correlated with their world and national rankings. Similar results were found for predictions about stroke force. However, the results showed that novices were also able to exploit some information in the pre-contact sequences in order to predict stroke force.

In addition to temporal occlusion, Abernethy (1990) employed spatial occlusion where the arm and racquet, racquet only, face and head, and lower body could be selectively occluded. In the expert players, prediction about stroke direction appeared to be unaffected by spatial occlusion; however, spatial occlusion did appear to affect the experts' predictions about stroke force. In particular, the results indicated a role for the arm as well as the face and head in determining the accuracy of predicted stroke force. Interestingly, the arm and the head might provide distinct types of information. For example, the arm might provide purely movement-based cues while the head might additionally provide cues such as where the player is attending and, therefore, intending on hitting the ball. (Unfortunately, this issue was not addressed by Abernethy, 1990). Recent work on anticipation in cricket batsmen has shown that observing the movement of the bowler's arm is a particularly important cue for generating predictions about how the ball will move once released from the bowler's hand (Müller, Abernethy, Eid, McBean, \& Rose, 2010). The importance of a body movement cue, such as arm movement, over cues such as body position is consistent with an account of action prediction based on action emulation. If action prediction is based on predicting the consequences of an action by emulating the dynamics of the action, then observation of movement dynamics should provide the most powerful cue for prediction.

In order to isolate the effects of movement kinematics from other visual information (e.g., eye gaze cues), the experiments of Sebanz and Shiffrar (2009) added a condition that employed point-light displays. In their task, subjects were required to make a judgement about whether a video of a basketball player performing a pass was performing a genuine 


\section{Introduction and background}

pass or a fake pass (the video was stopped before the ball was released). That is, they were asked to predict whether the movement shown would terminate with the ball being released. The results showed that expert basketball players were significantly more accurate at discriminating the genuine passes from the fake passes, suggesting that experts were able to exploit their motor expertise for the purposes of prediction. This effect was maintained even when subjects viewed point-light displays rather than videos, suggesting that kinematic cues are sufficient for prediction. In addition, the paradigm used by Sebanz and Shiffrar (2009) required subjects to generate a prediction about an actual movement, rather than merely the fate of an object hit or thrown by a person. Therefore, these results also show that prediction of actions, rather than only the consequences of an action (cf. Abernethy, 1990), might be influenced by motor expertise.

While these results appear to suggest that prediction accuracy may have a motor component, there are reasons to be cautious when drawing this conclusion. For example, it may be that experts are able to generate more accurate predictions merely because they have increased visual experience with observing the sport being played. One way to address this confound is to compare differences in prediction accuracy for performance experts (e.g., players) with observation experts (e.g., coaches and sports journalists). This was the approach adopted by Aglioti, Cesari, Romani, and Urgesi (2008). Their results showed that elite players, compared with novices and coaches, were significantly more accurate at judging whether a basketball thrown from the free throw position would enter the basket. Furthermore, coaches were no more accurate than novices at predicting the fate of the basketball. As with the results from tennis and squash players, experts were more accurate at predicting the fate of the ball even when they were not able to observe the ball in flight.

While comparisons of movement experts with observation experts goes some way to support the notion that motor expertise leads to enhanced prediction accuracy, there is still the question about the direction of the causal relationship. That is, it may be that elite athletes become skilled at prediction because of their movement skills, or they may 
become elite athletes because they already possess superior prediction abilities. One way to address this problem is to examine prediction ability as a function of the observer's kinematics. That is, are people more accurate at generating predictions about actions that are kinematically similar to their own actions? This can be achieved by employing a self/other prediction paradigm where people generate predictions about recordings of actions that were performed by other people as well as actions that they performed themselves.

Knoblich and Flach (2001) provide one of the first comparisons of prediction accuracy for self-produced and other-produced movements. They asked subjects to generate a prediction about the landing position of a thrown dart. Observers viewed a video of a thrower that contained information about the throwing action but excluded any information about the dart's flight trajectory. Furthermore, the movement sequences were presented under different levels of spatial occlusion: a full view condition without any occlusions; a body only condition, where the head was occluded and only body and arm were visible; and an arm only condition, where the body and the head were occluded. By restricting observers' access to information about the head and face it was possible to remove cues about where the dart thrower is attending (see Abernethy, 1990, cited above for evidence of gaze cue effects in action prediction). Similarly, body position might also provide cues about the locus of attention or the direction of the intended throw. However, in the case of the arm only condition, where gaze, head position, and posture cues are absent, subjects must rely on predicting the action consequences of the arm movement. Consistent with the findings of Abernethy (1990), subjects were most accurate at predicting the landing position of the dart when gaze and head position cues were present. When only the arm movement was visible subjects' predictions were least accurate, although still above chance levels. This suggests that while subjects are able to predict the landing position of the dart on the basis of the movement of the arm alone, the head provides additional non-redundant cues.

The crucial comparison, however, was the difference in accuracy for the predictions of self-produced dart throws compared with other-produced dart throws. The results 


\section{Introduction and background}

showed a general pattern of greater accuracy for self-produced dart throws. This self/other difference has been termed the authorship effect. However, this advantage was restricted to the latter half of the experiment. Interestingly, the pattern of results for the arm only condition did appear different, with a numerical difference between self-produced and other-produced dart throws appearing in the first experimental block; however, this difference was not statistically significant.

Taken together, it is possible to draw some conclusions from these results. First, the results suggest that observers are able to use limb movement cues in order to predict the consequences of an action. Second, they suggest that observers can learn to exploit knowledge of their own movement dynamics — that is, expertise in producing their own movements - in order to gain a prediction advantage. And last, the results suggest that when only limb movement cues are present, there is a possibility that observers may learn to exploit these movement cues more rapidly (as indicated by the numerical difference in the first block), possibly due to the absence of other cues (e.g., gaze cues) that might otherwise encourage a different strategy. Importantly, the results of Knoblich and Flach (2001) also highlight the importance of isolating kinematic cues, something that is difficult in the more naturalistic settings of the sport expertise studies cited earlier.

A subsequent study by Knoblich, Seigerschmidt, Flach, and Christensen (2002) was able to more precisely isolate the effects of movement kinematics. Unlike the study by Knoblich and Flach (2001), which employed video recordings, Knoblich et al. (2002) employed recordings of action-effects, namely recordings of pen traces. The experimental setup was as follows: In the first session, subjects were asked to write on a drawing tablet. They were required to produce a whole character or the initial stroke from that character in isolation. In a subsequent session, subjects observed a moving dot that traced the trajectory that had been produced earlier. Presented with only one stroke, the task was to decide whether the stroke had been produced in isolation or as part of a character. Participants performed significantly better for self-produced strokes than for other-produced strokes. Interestingly, Knoblich et al. (2002, Experiment 1) found that the self/other differences 
were reduced for well-practised characters relative to novel characters. The authors suggested that this might be because inter-subject variability is lower for well-practised actions, and the self/other difference relies on subtle differences between self-produced and other-produced actions. Inter-subject variability could also be reduced by constraining stroke production by employing guidelines that limited the characters' dimensions (Experiment 3), and this also reduced the size of the self/other difference.

While the studies outlined above provide some evidence for the involvement of the motor system in action prediction, (at least) three key questions remain unanswered. First, these studies do not examine whether people are capable of generating fine-grained predictions, because the prediction tasks can be performed without subjects ever generating a detailed prediction. For example, in the dart throwing task it may be the case that subjects are merely able to discriminate between darts thrown at various sections of the board on the basis of the kinematic properties of the action without ever generating a detailed prediction about the end point of the dart. Thus, coarse-grained predictions might be sufficient for performing the prediction tasks detailed above, but without more fine-grained details about the unfolding kinematics of the actions it is difficult to see how these predictions can be truly useful for joint action coordination. Second, the prediction tasks outlined above do not put a time constraint on predictions and, therefore, it is not clear how rapidly the output of these predictions becomes available to drive subsequent action planning. Joint action tasks such as joint music and dance performance may face heavy time constraints and, therefore, unless the output of the prediction system is available rapidly it is difficult to see what role it might play in supporting these activities. And third, the output of the action prediction mechanism must be available to action control. The studies outlined above asked subjects to verbally report their predictions and, therefore, it might be possible that different information, or no information at all, is available to action control.

The last question, whether any output from the action prediction mechanism is available to action control, is particularly important considering work by Milner and Goodale 


\section{Introduction and background}

(1996). This work has suggested that the visual system is divided into two distinct processing streams: a dorsal stream, which processes vision for action, and a ventral stream, which processes vision for perception. ${ }^{4}$ An illustration of this division can be seen in reports of the deficits suffered by a patient known as DF (Milner et al., 1991). DF suffered a profound deficit in visual recognition. She was unable to discriminate shapes or report the orientation of a slot cut into a disc. However, when DF was asked to "post" a card into the slot she was able to do so successfully. Thus DF's deficit appears to be restricted only for verbal reports about objects and her ability to interact with objects appears to be spared. This raises the possibility that in prediction tasks different information might be available for action control and verbal report.

A study by Mann, Abernethy, and Farrow (2010) has addressed this concern by employing a prediction task where subjects were either required to generate a verbal report of their prediction or to generate appropriate actions. Their task required cricket batsmen to observe a bowler as the bowler performed the action of bowling the ball. Balls bowled towards the batter can be directed either towards the left or the right of the batter (when the batter is directly facing the bowler) with the batter attempting to use the bat to hit the ball in a manner appropriate for the side that the ball was aimed at. The task required batters to judge whether a particular ball was directed toward the left or the right by responding in one of four ways: verbally reporting whether the ball was directed at the left or right without making any movements; moving the front foot into the correct position to play a shot; producing a full body movement to get into the correct position to play a shot, and playing the shot without a bat; or moving into position to play a shot, and playing the shot with a bat as they would do in a match. The results showed that expert batters were significantly more accurate at getting into the correct position to hit a ball directed at the left or right than they were at simply giving a verbal report about whether the ball was directed at the left or right. Interestingly, novices were just as accurate as ex-

\footnotetext{
${ }^{4}$ Recent work by Norman (2002) has attempted to map the dorsal stream and ventral stream onto ecological (e.g., Gibson) and constructivist theories of perception, respectively.
} 
perts during the verbal report condition, which suggests that the difference in skill level results in different information being available to the action control system, but not to conscious awareness. Furthermore, this study highlights the importance of the method of response used in prediction tasks. If the action prediction system is meant to underlie joint action, then it is important to assess whether the predictions generated are available for subsequent action planning. Verbal reports might, therefore, not be the correct method for assessing its function.

\subsubsection{Synchronisation}

One technique that can address all three questions-how rapidly predictions are available, whether they are fine-grained, and whether they are available to action control-is an action synchronisation task. Action synchronisation places a time constraint on the prediction process, which means that predictions must be available rapidly. Furthermore, these tasks require subjects to generate high-fidelity predictions that take into account fine-grained differences in the timing of synchronisation points between conditions. And finally, synchronisation tasks also require subjects to produce an action in response to the observed stimuli and, therefore, these tasks can assess whether information from the prediction system is available to action control.

The experiments reported by Flach, Knoblich, and Prinz (2003) take the first steps to addressing these issues. The self/other synchronisation task was split in to two parts with an initial movement phase followed by a synchronisation phase. In the movement phase, subjects were asked to draw up-and-down zigzags on a digitiser tablet while the dynamic trace was recorded. In the synchronisation phase, subjects viewed these dynamic traces and were asked to generate a prediction about the timing of particular synchronisation points by synchronising a button press with the occurrence of the synchronisation points. In this case, the synchronisation points were determined to be the points at which the dynamic trace changed from an upward movement to a downward movement. Synchronisation accuracy was measured as the arithmetic mean of the time difference between 


\section{Introduction and background}

the occurrence of the synchronisation points and the time at which the subject performed the button presses.

The results of the experiment showed that synchronisation accuracy was indeed greater for self-produced actions relative to other-produced actions. However, this difference (the authorship effect) did not emerge until the third and fourth block of the experiment. The delay in the emergence of the authorship effect is troublesome because it calls into question whether the action prediction system, which the authorship effect is supposed to index, is indeed used to support joint action coordination. Rather, this evidence suggests that there must be a second system that can be used for coordinating actions before the action prediction system comes on-line. Therefore, work still needs to be done to further elaborate the nature of the mechanisms that may underlie action prediction.

In addition to these experiments in the visual domain, experiments in the auditory domain by Keller, Knoblich, and Repp (2007) have reported similar findings. In their study, trained pianists were asked to play duets with either recordings of themselves or another person. The results showed superior timing accuracy when pianists played duets with themselves, as predicted by the emulator hypothesis. However, pianists are highly trained in auditory-motor synchronisation and, therefore, it is not clear how generalisable these effects are to non-experts. Therefore, it is important to examine the mechanisms of action prediction in non-experts.

\subsection{Aims of the current thesis}

The aim of the current thesis is to examine the nature of the mechanisms that underlie action prediction. Furthermore, this thesis aims to examine whether the nature of these mechanisms has implications for joint action and joint agency, and what these implications are. This thesis also aims to investigate whether the nature of the action prediction mechanism has implications for social phenomena beyond joint action. Specifically, the aim is to investigate whether our understanding of music and emotion can benefit from an understanding of action prediction mechanisms. 
The outline of the thesis is as follows: Part II aims to extend upon previous work using synchronisation paradigms and provides an empirical investigation of the action prediction mechanism. As suggested above, while there is some evidence in support of the idea that action prediction relies on a process of motor emulation several questions remain unresolved. Primary among these unresolved issues is the whether the output from these predictive processes is available to the action control system rapidly enough for it to be useful for subsequent action planning during joint action tasks. The experiment presented in Chapter 2 is designed to address this question. In particular, it examines whether the learning effects observed by Flach et al. (2003) persist even when subjects are presented with actual actions rather than action-effects. As this study is the first to employ actual actions in this type of action synchronisation task it also provides an important test of whether the authorship effect is generalisable to paradigms that use actions rather than action-effects.

The experiments presented in Chapter 3 are designed to examine the nature of the model employed by the action prediction system and the sources of information used to construct the model. In particular, it examines whether information about the limbs and joints performing an observed action facilitates the process of action emulation. Studies by Flach et al. (2003) have only used action-effects and not actual actions and, therefore, it has not been possible to examine what role information about the body producing the action plays in action synchronisation. The experiments presented in Chapter 3 use actual actions and systematically vary the amount of information available in the stimulus thereby making it possible to examine the influence of stimulus information. Furthermore, the experiments presented in Chapter 3 also examine the role of motor experience in action synchronisation. Previous studies have only used paradigms where subjects attempt to predict actions that they had themselves performed earlier. Therefore, it is not clear what the effect, if any, would be of using naïve subjects who do not have experience in producing the observed action.

Finally, in Chapter 4, an alternative explanation for the results from the action synchro- 


\section{Introduction and background}

nisation paradigm is examined. As previous studies of action synchronisation have used rhythmic stimuli this raises the possibility that the results might be explained through self-similarity in the rhythmic properties of the actions produced in the initial action production and subsequent action synchronisation phases of the experiments. The experiments presented in Chapter 4 are designed to rule out this alternative.

Part III (Chapter 5) provides a framework that integrates the findings of Part II with other empirical data on action co-representation and joint action coordination. Using these data, Part III argues that joint action coordination can be understood as a special case of individual action control. Furthermore, it develops an account of how the mechanisms responsible for joint action coordination might also be used to explain the phenomenology of joint action and feelings of group flow.

The aim of Part IV (Chapter 6) is to extend the ideas developed in the thesis beyond the realm of joint action coordination and into the realm of a different social phenomenon, namely music. Rather than being a purely acoustic phenomenon, Part IV argues that music is best understood as a type of action, with action observation playing an important role in musical experience. As argued in Part II and Part III, action observation engages predictive sensory-motor processes in the observer, and Part IV argues that these predictive sensory-motor processes may also be adept at explaining the expression of emotion in music by providing a means of unifying expectancy-based models and action-based models of emotional experience.

In this way, this thesis will demonstrate that social cognition is grounded in sensorymotor processes. To understand interpersonal interaction we must understand the interactions between perception and action.

\subsection{References}

Abernethy, B. (1990). Anticipation in squash: differences in advance cue utilization between expert and novice players. Journal of Sports Sciences, 8, 17-34. 
Aglioti, S. M., Cesari, P., Romani, M., \& Urgesi, C. (2008). Action anticipation and motor resonance in elite basketball players. Nature Neuroscience, 11(9), 1109-1116.

Boden, M. (2006). Mind as machine : a history of cognitive science. New York: Oxford University Press.

Calvo-Merino, B., Glaser, D. E., Grézes, J., Passingham, R. E., \& Haggard, P. (2005). Action observation and acquired motor skills: an fMRI study with expert dancers. Cerebral Cortex, 15(8), 1243-1249.

Clark, A. (1997). Being there: putting brain, body, and world together again. Cambridge MA: MIT Press.

Csibra, G. (2008). Action mirroring and action understanding: an alternative account. In P. Haggard, Y. Rossetti \& M. Kawato (Eds.), Sensorimotor foundations of higher cognition (pp. 435-459). New York: Oxford University Press.

Descartes, R. (1606/1998). Treatise on man. In S. Gaukroger (Ed. \& Trans.), The world and other writings (pp. 99-169). Cambridge: Cambridge University Press,

di Pellegrino, G., Fadiga, L., Fogassi, L., Gallese, V., \& Rizzolatti, G. (1992). Understanding motor events: a neurophysiological study. Experimental Brain Research, 91, 176-180.

Donders, F. C. (1869/1969). On the speed of mental processes. Acta Psychologica, 30, 412431.

Flach, R., Knoblich, G., \& Prinz, W. (2003). Off-line authorship effects in action perception. Brain and Cognition, 53(3), 503-513.

Gallese, V., \& Goldman, A. (1998). Mirror neurons and the simulation theory of mindreading. Trends in Cognitive Sciences, 2(12), 493-501.

Garbarini, F., \& Adenzato, M. (2004). At the root of embodied cognition: cognitive science meets neurophysiology. Brain and Cognition, 56(1), 100-106.

Gibson, J. J. (1966). The senses considered as perceptual systems. Boston: Houghton Mifflin. Gibson, J. J. (1986). The ecological approach to visual perception. New York: Psychology Press. 
Golnaraghi, M., \& Kuo, B. C. (2010). Automatic control systems (9th ed.). Hoboken NJ: Wiley.

Grush, R. (1997). The architecture of representation. Philosophical Psychology, 10(1), 523.

Grush, R. (2004). The emulation theory of representation: motor control, imagery, and perception. Behavioral and Brain Sciences, 27(3), 377-396.

Hedge, A., \& Marsh, N. W. A. (1975). The effect of irrelevant spatial correspondences on two-choice response-time. Acta Psychologica, 39(6), 427-439.

Hommel, B. (2009). Action control according to TEC (theory of event coding). Psychological Research, 73(4), 512-526.

Hommel, B., Müsseler, J., Aschersleben, G., \& Prinz, W. (2001). The theory of event coding: a framework for perception and action planning. Behavioral and Brain Sciences, 24(5), 849-878.

Iacoboni, M., Molnar-Szakacs, I., Gallese, V., Buccino, G., Mazziotta, J. C., \& Rizzolatti, G. (2005). Grasping the intentions of others with one's own mirror neuron system. PLoS Biology, 3(3), e79.

Isaacs, L. D., \& Finch, A. E. (1983). Anticipatory timing of beginning and intermediate tennis players. Perceptual and Motor Skills, 57(2), 451-454.

Jacob, P. (2008). What do mirror neurons contribute to human social cognition? Mind \& Language, 23(2), 190-223.

Keller, P. E., Knoblich, G., \& Repp, B. H. (2007). Pianists duet better when they play with themselves: on the possible role of action simulation in synchronization. Consciousness and Cognition, 16(1), 102-111.

Knoblich, G., \& Flach, R. (2001). Predicting the effects of actions: interactions of perception and action. Psychological Science, 12(6), 467-472.

Knoblich, G., Seigerschmidt, E., Flach, R., \& Christensen, W. (2002). Authorship effects in the prediction of handwriting strokes: evidence for action simulation during action 
perception. The Quarterly Journal of Experimental Psychology: Section A, 55(3), 10271046.

Mann, D. L., Abernethy, B., \& Farrow, D. (2010). Action specificity increases anticipatory performance and the expert advantage in natural interceptive tasks. Acta Psychologica, 135(1), 17-23.

Marr, D. (1982). Vision a computational investigation into the human representation and processing of visual information. San Francisco: W. H. Freeman.

Milner, A. D., \& Goodale, M. A. (1996). The visual brain in action. Oxford: Oxford University Press.

Milner, A. D., Perrett, D. I., Johnston, R. S., Benson, P. J., Jordan, T. R., Heeley, D. W., ... Terazzi, E. (1991). Perception and action in 'visual form agnosia'. Brain, 114, 405428.

Müller, S., Abernethy, B., Eid, M., McBean, R., \& Rose, M. (2010). Expertise and the spatiotemporal characteristics of anticipatory information pick-up from complex movement patterns. Perception, 39(6), 745-760.

Müsseler, J., \& Hommel, B. (1997). Blindness to response-compatible stimuli. Journal of Experimental Psychology: Human Perception and Performance, 23, 861-872.

Newell, A. (1994). Unified theories of cognition. Cambridge MA: Harvard University Press.

Newell, A., \& Simon, H. A. (1961). Computer simulation of human thinking. Science, 134, 2011-2017.

Newell, A., \& Simon, H. A. (1997). Computer science as empirical inquiry: symbols and search. In J. Haugeland (Ed.), Mind design II: philosophy, psychology, artificial intelligence (pp. 81-110). Cambridge MA: MIT Press.

Norman, J. (2002). Two visual systems and two theories of perception: an attempt to reconcile the constructivist and ecological approaches. Behavioral and Brain Sciences, 25(1), 73-96.

Prinz, W. (1997a). Perception and action planning. European Journal of Cognitive Psychology, 9(2), 129. 
Prinz, W. (1997b). Why Donders has led us astray. In B. Hommel \& W. Prinz (Eds.), Theoretical issues in stimulus-response compatibility (pp. 247-267).

Repp, B. H., \& Knoblich, G. (2007). Action can affect auditory perception. Psychological Science, 18(1), 6-7.

Rizzolatti, G., Camarda, R., Fogassi, L., Gentilucci, M., Luppino, G., \& Matelli, M. (1988). Functional organization of inferior area 6 in the macaque monkey. Experimental Brain Research, 71, 491-507.

Rizzolatti, G., Fogassi, L., \& Gallese, V. (2001). Neurophysiological mechanisms underlying the understanding and imitation of action. Nature Reviews: Neuroscience, 2(9), 661-670.

Sebanz, N., \& Shiffrar, M. (2009). Detecting deception in a bluffing body: the role of expertise. Psychonomic Bulletin \& Review, 16(1), 170-175.

Shiffrar, M., \& Freyd, J. J. (1990). Apparent motion of the human body. Psychological Science, 1(4), $257-264$.

Simon, J. R. (1969). Reactions toward the source of stimulation. Journal of Experimental Psychology, 81(1), 174-176.

Sternberg, S. (1969). The discovery of processing stages: extensions of Donders' method. Acta Psychologica, 30, 276-315.

Umiltà, M. A., Kohler, E., Gallese, V., Fogassi, L., Fadiga, L., Keysers, C., \& Rizzolatti, G. (2001). I know what you are doing: a neurophysiological study. Neuron, 31(1), 155165.

Varela, F., Thompson, E. T., \& Rosch, E. (1991). The embodied mind cognitive science and human experience. Cambridge MA: MIT Press.

Viviani, P., \& Stucchi, N. (1992). Biological movements look uniform: evidence of motorperceptual interactions. Journal of Experimental Psychology: Human Perception and Performance, 18(3), 603-623.

Viviani, P., \& Terzuolo, C. (1982). Trajectory determines movement dynamics. Neuroscience, $7(2), 431-437$. 
Wilson, M. (2002). Six views of embodied cognition. Psychonomic Bulletin \& Review, 9(4), $625-636$.

Wilson, M., \& Knoblich, G. (2005). The case for motor involvement in perceiving conspecifics. Psychological Bulletin, 131(3), 460-473.

Wolpert, D. M. (1997). Computational approaches to motor control. Trends in Cognitive Sciences, 1(6), 209-216.

Wolpert, D. M., \& Kawato, M. (1998). Multiple paired forward and inverse models for motor control. Neural Networks, 11(7-8), 1317-1329. 



\title{
Part II.
}

An empirical investigation of action

\author{
prediction
}





\section{CHAPTER 2}

\section{Action synchronisation with biological motion}

\subsection{Introduction}

In order to engage in joint action it is crucially important that agents are able to coordinate their actions with each other. Humans possess an astonishing ability for highly accurate temporal coordination of action. This ability for temporally coordinated, or synchronised, action is evident in pursuits such as music and dance, where synchronised action can be an important part of the aesthetic qualities of the performance. That humans can synchronise their actions with great accuracy is not in doubt; however, what is less clear is the mechanism that underlies this ability.

Synchronisation tasks impose heavy time demands on action execution. To meet these time demands, actions need to be planned early so that execution can be temporally synchronised. Therefore, it is unlikely that actions are planned based on the incoming sensory input because sensory transduction would introduce disruptive time delays. To bypass these delays, and to achieve the temporal precision that is evident in many synchronisation tasks, it is necessary for actors to anticipate the movements of their co-actors. This 


\section{Action synchronisation with biological motion}

may be done by predicting how their co-actor might move. The results of these predictions would be available to the actor's action system before sensory input from the outside world and could, therefore, be used as the basis for early, or anticipatory, motor planning.

\subsubsection{Prediction}

Anticipatory motor planning, and prediction, has been extensively studied within the context of sensorimotor synchronisation (SMS) tasks. These tasks often take the form of finger tapping in time with a regular beat (for a review, see Repp, 2005). In order to achieve synchronisation in this type of task it is necessary to form a representation of the temporal structure of the stimulus so that the appropriate motor command can be issued before the next beat occurs. That is, it is necessary to take the current stream of sensory information and predict the future stream of sensory information. Recent neurophysiological evidence suggests a crucial role for the cerebellum in SMS tasks. This is unsurprising considering the role that the cerebellum plays in prediction, particularly during motor control (Miall, Weir, Wolpert, \& Stein, 1993).

Models of cerebellar function suggest that a core function of the cerebellum is to implement a forward, or predictive, model (Miall et al., 1993). Forward models use the current state of a system as input and from this they predict the future state of the system. This type of prediction may be particularly important during action control where the current state of an effector, along with the motor command, is used to predict the proprioceptive feedback that would be generated by a particular movement. This predicted proprioceptive feedback is thought to stand in for actual sensory feedback thereby bypassing sensory transmission delays (for a review, see Wolpert, Miall, \& Kawato, 1998). In SMS tasks, forward models may also play a similar role in anticipating future sensory events by taking the incoming stream of sensory events (beats) and projecting it forward in time (Tesche \& Karhu, 2000). 


\subsubsection{Predicting human action}

SMS tasks, such as tapping to the beat, require people to predict temporal properties of stimuli that are often relatively artificial. There has only been limited work to extend the SMS paradigm into more naturalistic settings. For example, Repp and Keller (2008) employed a tapping paradigm that simulated tapping in time with another human participant. This use of more human-like stimuli is important because there is increasing evidence that stimuli generated by human movement are perceptually special. Moreover, it has been shown that the motor laws that govern the production of movements also influence how these movements are subsequently perceived. For example, the two-thirds power law relates the speed at which a curved trajectory is drawn to the curvature of the trajectory-that is, it is a law governing action production (Viviani \& Terzuolo, 1982). This law, however, also influences perceptual tasks. For example, Viviani and Stucchi (1992) found that when participants viewed pen traces producing an ellipse, and were asked to adjust the velocity profile of the traces so that they appeared to move around the ellipse at a constant speed, participants made systematic errors and selected velocity profiles that were consistent with the two-thirds power law. Thus, for these stimuli, there is evidence that motor processes "leak" into, or affect, perceptual processes.

The two-thirds power law also influences the ability to generate predictions about the stimuli. Kandel, Orliaguet, and Viviani (2000) asked participants to view excerpts of pen trajectories that were instances of either the letter combination "lll" or "lln" written in a cursive style. When shown only the middle letter, and then asked to predict whether the following letter would be an "l" or an " $\mathrm{n}$ ", observers were able to perform this task with reasonable accuracy (approximately 69\% correct). When the trajectories were altered, however, so that the velocity profile of the trajectory no longer approximated real handwriting (that is, when it no longer obeyed the two-thirds power law) the accuracy with which the last letter could be predicted dropped markedly.

It is unsurprising that motor processes can influence perceptual processes for humanmovement produced stimuli considering neurophysiological evidence suggests a tight link 


\section{Action synchronisation with biological motion}

between perceptual and motor processes. In particular, mirror neurons and canonical neurons in premotor cortex have been shown to have both perceptual and motor properties (for a review, see Gallese, Fadiga, Fogassi, \& Rizzolatti, 1996). The mirror-neuron system transforms perceptual information into motor information by being active both when a particular action is performed and when that action is observed (or when a sound associated with the action is heard; Kohler et al., 2002). This suggests that there may be a common neural code for the production and perception of actions. A number of proposals have been put forward for the role that mirror neurons might play in human cognition. Most prominent among these is the notion that mirror neurons might be the neural substrate of third person mind reading (e.g., Gallese \& Goldman, 1998). However, some have criticised this view, and an alternative view, that mirror neurons might underlie the more basic function of predicting the actions of other agents, has been suggested (Jacob, 2008; Wilson \& Knoblich, 2005).

\subsubsection{The emulator model of human action prediction}

Wilson and Knoblich (2005) have suggested that mirror neurons form part of an emulator, or forward model (Grush, 2004), that is used to predict the movements of other agents. They suggest that the emulator generates a real-time simulation of the movements of other agents, and these simulations, which run concurrently with sensory input, are then used to predict the unfolding trajectory of an observed motor act. These predictions are able to stand in for actual sensory input bypassing delays that would be introduced by waiting for actual sensory information to arrive. Therefore, predicted input can serve as the basis for early, or anticipatory, motor planning. It is unlikely that the emulator is instantiated in a purely perceptual system because of the computational complexity of predicting human movement. This computational challenge may be greatly reduced if the emulator piggybacks on the motor system, by way of mirror neurons, thus allowing the emulator to exploit existing knowledge of human movement dynamics that is embodied in the motor system. 
If it is the case that knowledge embodied in the observer's motor system is used as the basis for predicting the actions of other agents, then idiosyncrasies of the observer's motor system should "leak out" into the predictions that they generate. It follows that the emulator hypothesis would predict superior prediction accuracy when observers generate predictions about the movements of agents that move similarly to themselves. That is, if observers use knowledge of their own motor dynamics to generate predictions about how other agents will move, then a better match between the movement dynamics of the observer and the observed agent will result in enhanced prediction accuracy. This should be most evident when observers generate predictions about their own (recorded) movements because in this case there is a perfect match between the dynamics of the predicting system and the predicted system. This hypothesis has found support in several studies using a self/other prediction paradigm.

The self/other prediction paradigm employed by Knoblich and Flach (2001) required observers to generate a prediction about the landing position of a dart that was thrown at a dartboard. Observers were shown a series of video recordings depicting both the observer and another person throwing darts towards a dartboard. The recordings were stopped at the point where the dart was released from the hand, and the observer then had to generate a prediction about the landing position. Observers were significantly better at predicting the dart landing position from video recordings of themselves compared to recordings of other people. A similar experiment by Knoblich, Seigerschmidt, Flach, and Christensen (2002) asked participants to generate a prediction about whether a pen stroke was produced in isolation or as part of a character. Once again, predictions were more accurate when observers viewed their own handwriting.

The studies outlined above are consistent with a role for motor emulation in action prediction; however, these studies employed an off-line (or observe then predict) prediction paradigm where participants generated their prediction off-line after viewing the stimulus. It is still not clear how rapidly the information from the emulator is available to use for on-line action control. To assess this, Flach, Knoblich, and Prinz (2003) employed a 

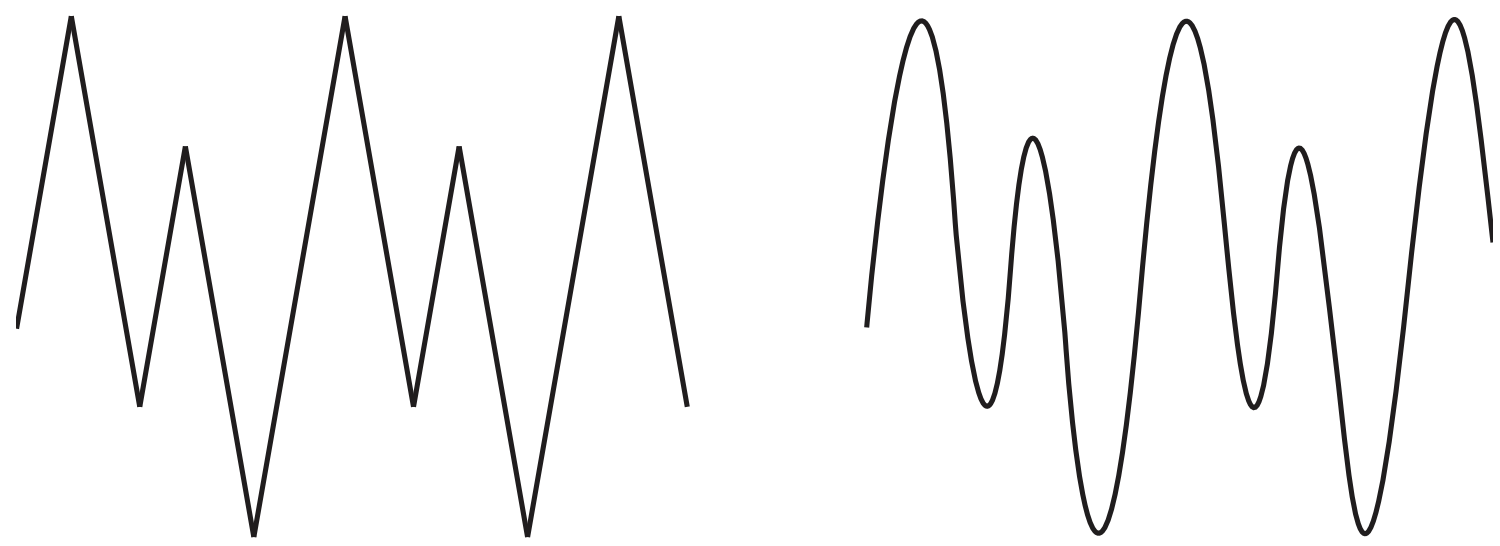

Figure 2.1. The zigzag (left) and wave (right) patterns used as stimuli during the recording session.

self/other synchronisation task. This task required participants to observe a moving dot as it traced a zigzag or wave trajectory (cf. Figure 2.1), and to press a response button when the moving dot reached the apex of each peak. These trajectories had been generated earlier by asking participants to draw them on a digitiser tablet. During the test session, participants were presented with trajectories that they had produced themselves as well as trajectories that had been produced by another person. As predicted by the emulator hypothesis, superior prediction accuracy (as measured by synchronisation error) was observed for self-produced trajectories over other-produced trajectories. However, this self-prediction advantage, or authorship effect, only emerged in the final two (of four) experimental blocks.

The delay in the onset of the authorship effect, observed by Flach et al. (2003), is surprising because the results of Knoblich and Flach (2001, Group 3) suggest that the authorship effect can emerge more rapidly. The most salient difference between Knoblich and Flach (2001) and Flach et al. (2003) is that Knoblich and Flach (2001) presented observers with actual bodily information (information about the limbs and joints) whereas Flach et al. (2003) only presented observers with the consequences of human movement, namely pen traces. It may take time for observers to map these motor consequences onto their own action system before they can exploit the knowledge embodied in their action system 
for the purposes of synchronisation. If this hypothesis is correct, then it may be possible to achieve an immediate authorship effect by presenting bodily movement to observers.

\subsubsection{Aims and hypotheses}

The primary aim of the present study is to replicate the findings of Flach et al. (2003) which demonstrate that observers use knowledge embodied in their own action system in order to predict how other agents will move. Furthermore, we aim to extend these findings by using stimuli that include actual bodily movements rather than stimuli that merely show the consequences of human movements. To this end, we employ moving animations of 3-D characters as stimuli. We anticipate that prediction accuracy (as measured by synchronisation accuracy) will be enhanced when observers synchronise with 3-D characters constructed from self-generated movements compared with characters constructed from other-generated movements. Furthermore, we predict that the inclusion of actual bodily information (in the form of limbs and joints) will result in an immediate authorship effect.

\subsection{Methods and materials}

\subsubsection{Participants}

Twenty-two participants (18 females), with an average age of 24 years (range $=18$ to 49 years), took part in the experiment. Twenty-one participants were right handed as assessed by the Edinburgh Handedness Inventory (Oldfield, 1971). All participants had normal or corrected-to-normal vision and were free of movement difficulties. All procedures were approved by the Macquarie University Human Subjects Ethics Committee.

\subsubsection{Motion capture}

Motion data was recorded using an 8-camera 3-D passive optical motion capture system (Vicon MX+ with 4 Vicon MX-F20 and 4 Vicon MX13+ cameras) at a sampling rate of 

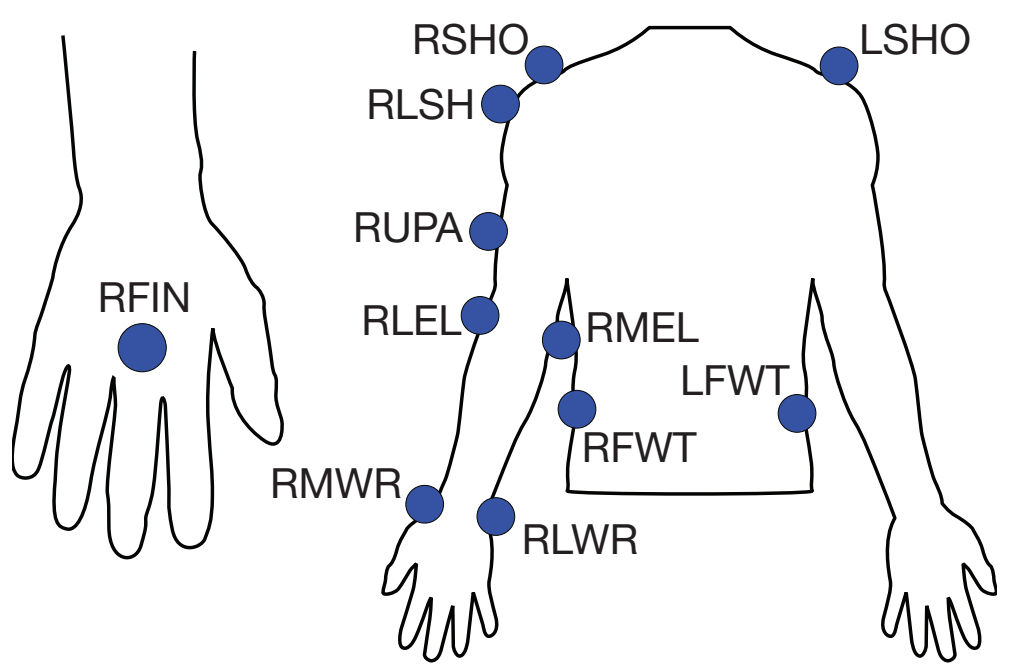

Figure 2.2. Marker positions for the 11 reflective markers used during the recording session.

$200 \mathrm{~Hz}$. A total of 11 spherical markers measuring $9 \mathrm{~mm}$ in diameter were placed on the shoulders, right arm, right hand, and waist of the participant (see Figure 2.2). The shoulder markers were placed on the top of the left shoulder (LSHO) and right shoulder (RSHO) and on the lateral side of the right shoulder (RLSH). Right arm markers were placed on the lateral side of the right upper arm (RUPA) and on the inside (RMEL) and outside (RLEL) elbow. Right hand markers were placed on the medial wrist (RMWR) and lateral wrist (RLWR) and at the base of the right middle finger (RFIN). Waist markers were placed between the iliac crest and the rib cage on the left (LFWT) and right (RFWT) side. Participants were instructed to keep their left arm rested by their side to ensure that it did not obscure the left waist marker. Participants were also instructed to clench their fist to ensure that the fingers did not move independently of the rest of the hand.

\subsubsection{Stimuli}

\subsubsection{Recording session}

Participants reproduced two movement patterns (Figure 2.1, adapted from Flach et al., 2003) during the recording session. These movement patterns were chosen so that the 
results of the present study would be more directly comparable to those of previous studies (i.e., Flach et al., 2003). Both patterns consisted of five peaks and five troughs with the peaks alternating in height. The two patterns differed, however, in the nature of the direction change at the apex of the peaks. The zigzag pattern had a sharp direction change while the wave pattern had a smooth, flowing, direction change. Participants viewed the patterns on large sheets of cardboard measuring $0.594 \mathrm{~m} \times 0.841 \mathrm{~m}$. For the zigzag pattern, the first up-stroke was $0.3 \mathrm{~m}$ long, with the remaining short up-strokes measuring $0.25 \mathrm{~m}$ and the long up-strokes measuring $0.5 \mathrm{~m}$. All the down-strokes were $0.375 \mathrm{~m}$ long. The angles enclosed by the strokes measured 0.35 radians. The wave pattern was based on the zigzag pattern and the stroke lengths were closely matched. The eccentricity of the curve at the apex was approximately 0.95 for all peaks.

\subsubsection{Test Session}

Raw motion capture data was first resampled to $25 \mathrm{~Hz}$. Animated characters were constructed with C-Motion Visual 3D (C-Motion INC, Rockville MD) using the resampled motion data. The characters consisted of an upper torso and right arm and hand. The torso was constructed using the shoulder markers (RSHO and LSHO) and waist markers (RFWT and LFWT). The upper arm was constructed using the shoulder markers and elbow markers (RMEL and RLEL), and the right lower arm was constructed using the elbow and wrist markers (RMWR and RLWR). The hand was constructed using the wrist markers as the proximal endpoint and the right finger marker (RFIN) as the midpoint. The size of the torso was not accurately modelled for each participant; however, the limb lengths were modelled using measurements acquired from the captured marker positions. Thus, limb lengths appeared relatively longer for animations constructed from taller participants and shorter for animations constructed from shorter participants. All animated characters were presented from a frontal view so that the action appeared as if viewed from the front (see Figure 2.3). 


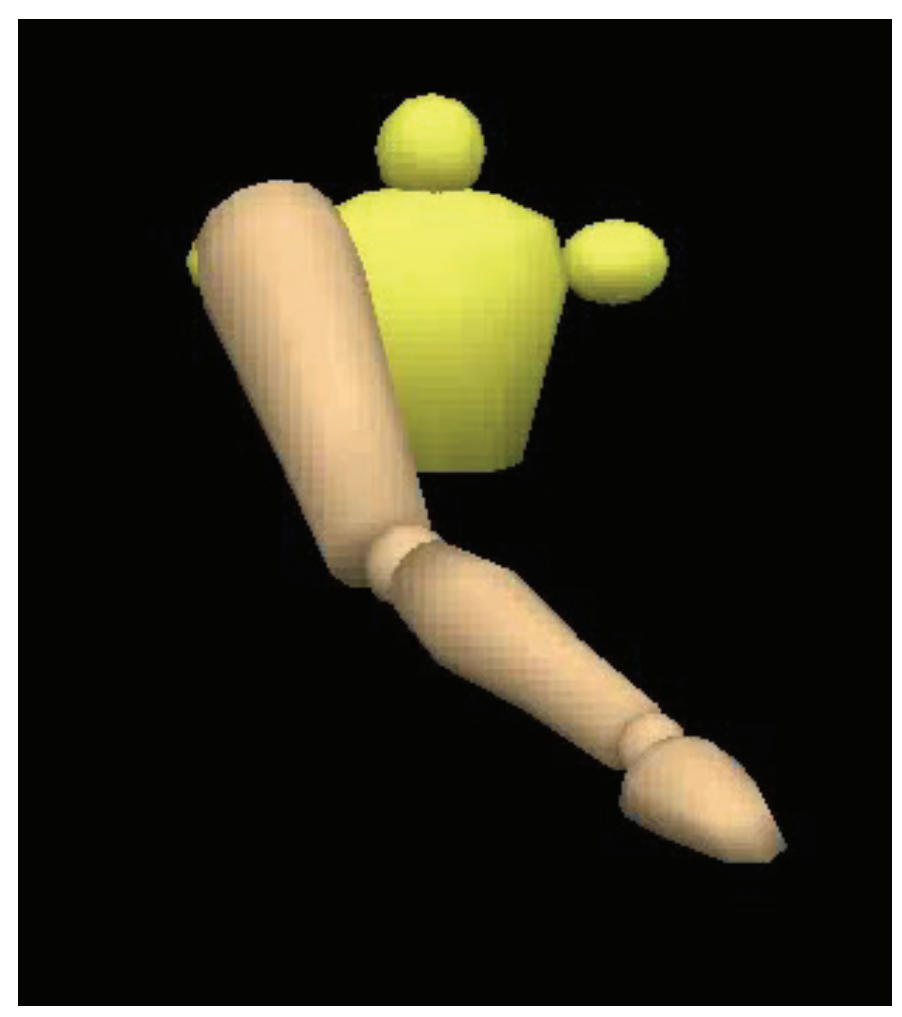

Figure 2.3. Sample of the 3-D animated character used during the test session.

\subsubsection{Procedure}

The experiment was split over two sessions separated by approximately 2-6 weeks $(M=18$ days, $S D=7$ ). The delay was introduced to control for possible effects of episodic memory recall. During the recording session, participants sat on a chair in the middle of the capture volume. At the start of each trial, they were shown the appropriate pattern and were told to produce either a wave or a zigzag. To generate the movements, participants were asked to pretend that they were drawing the patterns on a large blackboard. This involved making a series of up-and-down arm movements. All participants produced the movements with their right hand. Participants practised each pattern once with their eyes open and then reproduced each pattern 15 times. Participants performed these movements with their eyes closed to control for visual familiarity with the movement. The movements were produced in 3 blocks containing 5 repetitions of each pattern in a randomised order. Participants reproduced the patterns at their own pace (see Section 2.2.5 for details of inter-subject variability). 
In the test session, participants observed a series of animated 3-D characters (see Figure 2.3). Animations were used in order to control for the possible effects of overt recognition cues. The movement of the characters was controlled by the motion capture data recorded during the recording session. To ensure that stimulus factors did not influence synchronisation accuracy, participants were grouped into randomly assigned pairs, and each participant observed a series of animated characters controlled by their own movement data as well as a series of animated characters controlled by the movement data of the other participant in the pair. Both participants in the pair viewed the same set of animations in identical order. The task in the test session was to press the response button when the hand of the character reached the peak of each upward movement. Participants were instructed to synchronise the button-press with the display as accurately as possible and were told that this may require them to anticipate when the peak will occur. Each participant performed 4 blocks containing 40 unique stimuli (20 trials of self-generated movements and 20 trials of other-generated movements). The stimuli were presented in a pseudo-random order so that self-generated movements and other-generated movements were evenly distributed through the block.

\subsubsection{Movement description}

The length of each movement (distance travelled by the RFIN marker) was on average $4.051 \mathrm{~m}(S D=0.570)$ with participant averages ranging between $3.208 \mathrm{~m}$ and $5.487 \mathrm{~m}$. The average length of the long up-strokes was $0.441 \mathrm{~m}(S D=0.066$; range $=0.611$ to $0.355 \mathrm{~m})$, and the average length of the short up-strokes was $0.315 \mathrm{~m}(S D=0.061$; range = 0.435 to $0.230 \mathrm{~m})$. The average length of the down-strokes was $0.414 \mathrm{~m}(S D=0.060$; range $=0.557$ to $0.332 \mathrm{~m})$. The average duration of each movement was $6.86 \mathrm{~s}(S D=$ 1.74) with participant averages ranging between $3.37 \mathrm{~s}$ and $10.05 \mathrm{~s}$. All movements were completed in less than $12 \mathrm{~s}$. The average up-stroke duration was $0.66 \mathrm{~s}(S D=0.16)$ for the long up-strokes and $0.59 \mathrm{~s}(S D=0.16)$ for the short up-strokes. The average duration for the down-strokes was $0.71 \mathrm{~s}(S D=0.19)$. 


\section{Action synchronisation with biological motion}

\subsection{Results}

The peak of each upward movement was determined using the raw motion capture data. The movement leading up to the first peak was often contaminated with artifact and, therefore, the first peak was dropped from analysis. Timing error was calculated as the absolute difference between the timing of the peak in the motion capture data and the timing of the button-press. Absolute timing error was used because it provides a good proxy for accuracy (Spray, 1986). Button presses with a timing error that was greater than $250 \mathrm{~ms}$ were excluded from analysis. This was done to ensure that participants were anticipating the peak rather than merely responding to the peak once it had appeared on screen. This resulted in approximately $9.8 \%$ of button-presses being dropped from analysis (including all button-presses did not change the pattern of results reported below). Data analysis was performed on absolute timing error using a $2 \times 4$ repeated measures ANOVA with the factors Authorship (self, other) and Block (1, 2, 3, and 4). The Greenhouse-Geisser procedure was used to correct for violations of sphericity, where appropriate (we report uncorrected $d f$ s along with the $\epsilon$ value).

We found a significant main effect of Authorship $\left(F_{1,21}=6.838, p=.021, \eta_{p}^{2}=.230\right)$ indicating that timing error was significantly lower when synchronising with recordings of self-generated actions $(M=95.19 \mathrm{~ms}, S D=16.21)$ compared to other-generated actions $(M=103.14 \mathrm{~ms}, S D=19.15)$. The authorship effect, calculated as the difference in timing error between other-generated and self-generated actions, was positive (i.e., higher timing error for other-generated actions) for 10 of the 11 participant pairs $\left(M_{\text {diff }}=7.95\right.$, $95 \% \mathrm{CI}=1.35$ to 14.55$)$. The main effect for Block $\left(F_{3,63}=0.354, p=.676, \epsilon=.585\right.$, $\left.\eta_{p}^{2}=.017\right)$ and the Block $\times$ Authorship interaction did not reach significance $\left(F_{3,63}=1.708\right.$, $\left.p=.174, \eta_{p}^{2}=.075\right)$, indicating that both the timing error and the size of the authorship effect did not vary systematically across the four experimental blocks. These data are shown in Figure 2.4.

Previous studies (for a review, see Fraisse, 1982) have suggested that people have a preferred tempo for producing rhythmic movements such as the arm movements and the 


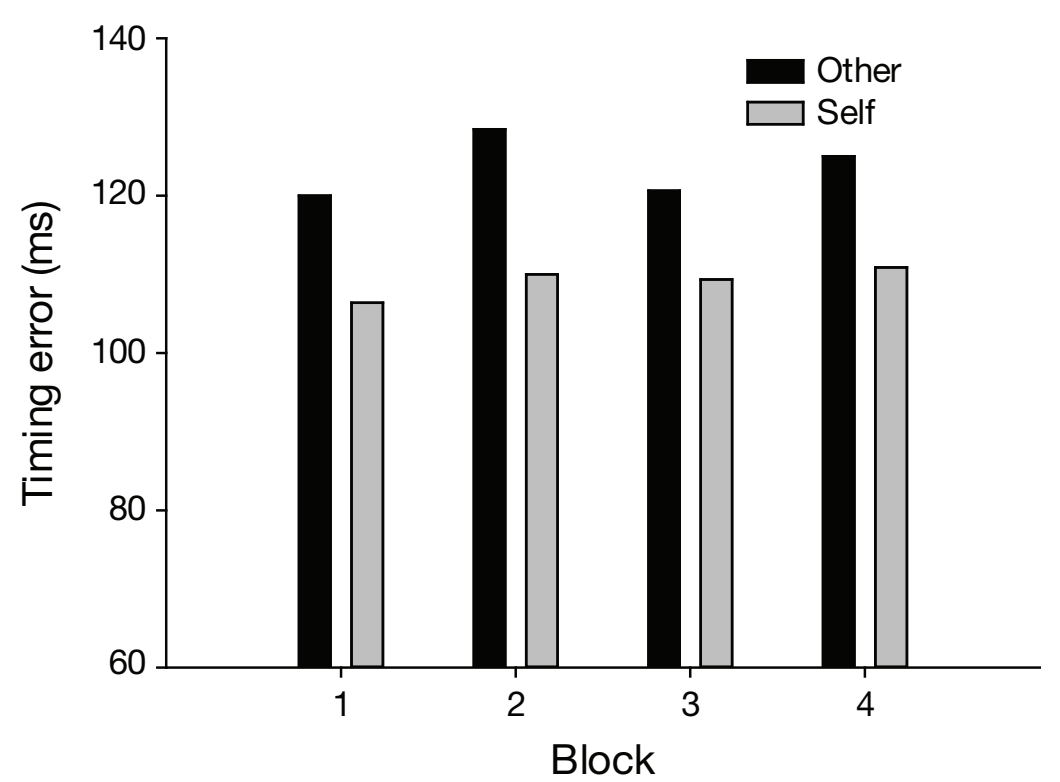

Figure 2.4. Mean timing error for self-generated and other-generated actions.

button-press movements in the present study. Therefore, it is possible that the authorship effect is not related to motor emulation but is dependent on there being a difference in the preferred tempo between the two participants in the pair. To test this possibility, we examined the relationship between the size of the authorship effect and the tempo similarity of the two participants in the pair. Tempo similarity was measured as the absolute difference in average tempo (measured in peaks per minute) for the arm movement recordings from each participant in the pair. No reliable relationship was found between the size of the authorship effect and tempo similarity $(r=-.040, p=.909)$. These data are shown in Figure 2.5 (left).

The results of Flach et al. (2003) have suggested that the authorship effect is dependent on peak irregularity - an authorship effect was not found for movements where the peak height did not alternate between large and small. To test for an effect of peak irregularity in the present data, we examined the relationship between the size of the authorship effect and peak irregularity (measured as the absolute difference between the average length of the large up-strokes and the average length of the small up-strokes). An increase in peak irregularity was associated with an increased authorship effect (see Figure 2.5 right). However, this relationship only approached significance $(r=.548, p=.081)$. 

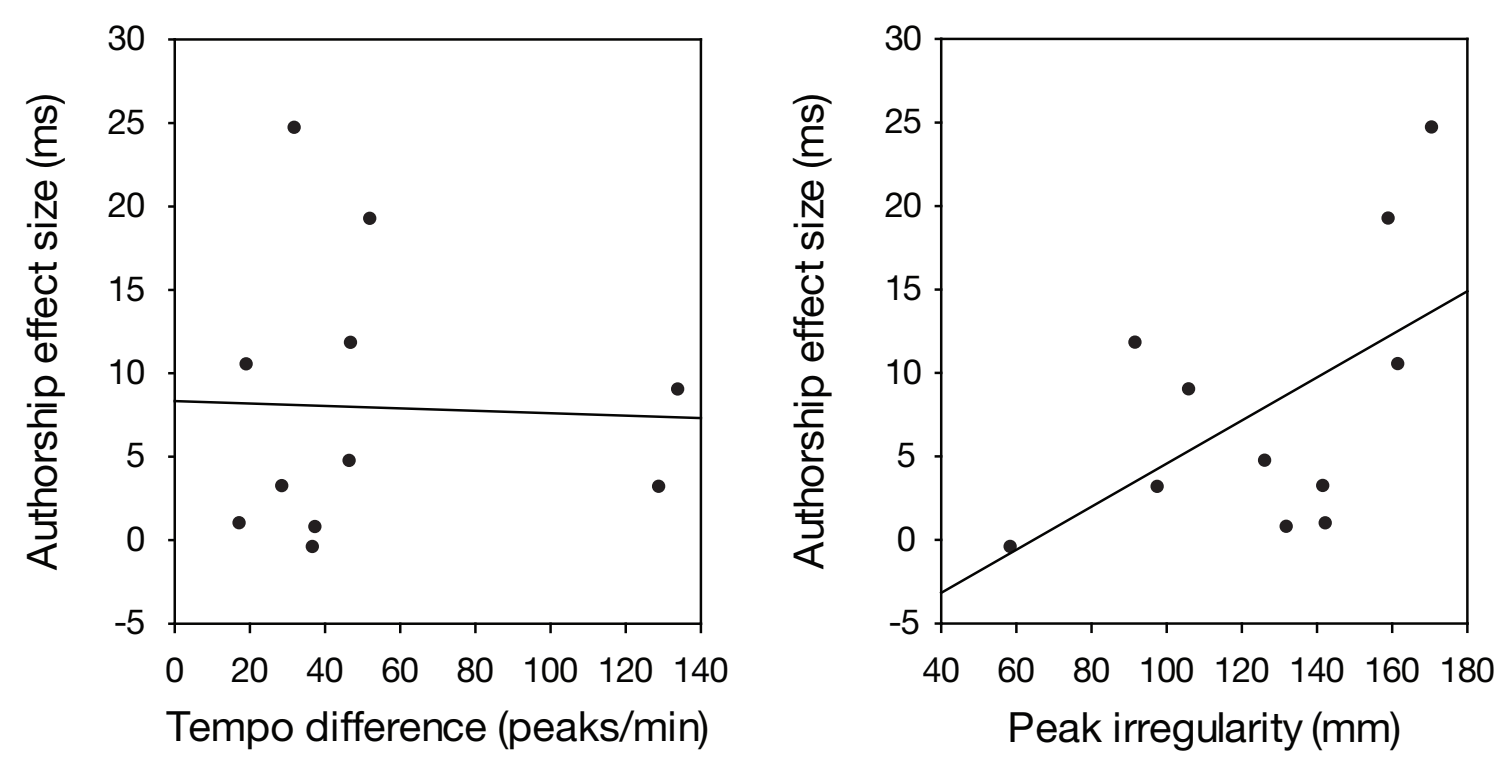

Figure 2.5. The relationship between the size of the authorship effect and tempo difference (left) and the size of the authorship effect and peak irregularity (right).

\subsection{Discussion}

The primary aim of the present study was to investigate the mechanisms underlying interpersonal action prediction. In particular, we tested the hypothesis that observers use knowledge embodied in their own action system to generate predictions about the movements of other agents. Our results are consistent with the hypothesis that prediction accuracy is dependent on the match between the observer's and observed agent's action systems.

\subsubsection{Authorship effect}

In order to measure the degree to which the observer's action system influenced their ability to predict the actions of other agents, we measured prediction accuracy (measured as synchronisation accuracy) for displays of self-generated and other-generated actions. The logic being that if the observer used knowledge embodied in their own action system to predict the actions of other agents then prediction accuracy should be enhanced when there is a perfect match between the system used for predicting and the system be- 
ing predicted - that is, when observing recordings of self-generated action. As predicted, synchronisation accuracy was enhanced when participants synchronised with recordings of self-generated actions compared with recordings of other-generated actions. This is consistent with the idea that observers use their own motor dynamics as the basis for action prediction. Our findings are consistent with previous experiments using similar stimuli (Flach et al., 2003).

An alternative explanation for the results is that participants found it easier to synchronise with recordings of self-generated actions because these actions were produced at a comfortable tempo for producing button-presses. Rather than attempting to predict the peak of each upward movement, participants may have merely produced buttonpresses at a tempo comfortable for them, and synchronisation accuracy was enhanced due to the coincidence between their preferred tempo for producing arm movements and their preferred tempo for producing button-presses. Several studies (for a review, see Fraisse, 1982) have suggested that people do indeed have a preferred tempo for spontaneous rhythmic movement. Because we did not control for production tempo, it is possible that participants produced arm movements at their preferred tempo. Furthermore, the use of regular rhythmic stimuli may have enhanced the tendency to produce movements at a preferred tempo. Synchronisation accuracy for self-generated actions may have been enhanced merely by participants reproducing their own preferred tempo during the test session. We attempted to rule out this possibility by examining the relationship between the size of the self-synchronisation advantage (or authorship effect) for the two participants in the pair and the similarity between arm movement tempo for the two participants in the pair. If these results could be explained by preferred tempo then we would expect to find that the authorship effect increased as the difference in arm movement tempo increased. This, however, was not the case, as there was no systematic relationship between tempo difference and the size of the authorship effect.

We also tested for a relationship between the size of the authorship effect and the regularity of the peak heights. Flach et al. (2003) found that the authorship effect was only 


\section{Action synchronisation with biological motion}

present when participants synchronised with irregular movements but not with regular movements. The authors suggested that when the movements were regular the participants could use the height of the preceding peak as a prediction cue for the height of the following peak. Therefore, the height of the following peak could be predicted without the need to rely on knowledge embodied in the observer's action system.

In the case of irregular movements, however, the height of the preceding peak cannot be used as a cue for the height of the following peak. Therefore, participants may need to make use of knowledge embodied in their own action system (that is, motor emulation) in order to predict the height of the following peak. The results of the present study are suggestive of a relationship between movement irregularity and the size of the authorship effect with the authorship effect increasing as movements become more irregular. The alternative hypothesis - that enhanced synchronisation accuracy is due to the coincidence of idiosyncratic tempo-would not predict any effect of peak irregularity. Future experiments will need to explicitly rule out this alternative explanation by either explicitly controlling for production tempo or by using non-rhythmic stimuli.

A further alternative explanation for the results presented here is that superior timing accuracy was observed for self-generated movements because participants had more visual experience with their own movements. On this account, the authorship effect would be due to purely perceptual processes. However, the up-and-down arm movements employed in our task are unlikely to be movements that are commonly performed and, therefore, it is unlikely that participants have acquired visual experience with them. Furthermore, during the recording session, participants performed the movements with their eyes closed ruling out the possibility of acquiring visual experience during the experiment. It is more plausible that participants acquired motor experience during the recording session, and the use of this motor knowledge for action prediction is consistent with the emulator hypothesis. 


\subsubsection{Block effect}

Flach et al. (2003, Experiment 2), in a study measuring synchronisation accuracy with up-and-down pen traces, found no difference between synchronisation accuracy for selfgenerated and other-generated actions during the first two experimental blocks. Only during the third and fourth experimental blocks did the authorship effect emerge. In the present study, however, there was no systematic change in the size of the authorship effect across the four experimental blocks. The most salient difference between Flach et al. (2003) and the present study is that Flach et al. (2003) did not present participants with actual human movement. The pen traces used as stimuli in this study were representations of the results or consequences of human movement. The present study, however, presented participants with actual limb movements by using 3-D animated characters composed of a torso and right arm. Stimuli of this type are isomorphic with the observer and, therefore, may be more easily mapped onto the observer's body. Before the emulator system can be used for action prediction, the emulator needs information about which effectors to model, and providing this information in the stimulus may facilitate building an appropriate model. The somatatopic organisation of the mirror-neuron system (Buccino et al., 2001) suggests that stimuli of this type may lead to increased mirror neuron activation in the observer. This increase in somatatopic activation may be what is providing an increase in information about the initial parameter settings (e.g., which limbs to model) to the observer's emulator system and this may enhance motor emulation. The authorship effect arises because the emulator uses the observer's own motor dynamics to model the observed agent and, therefore, enhancing motor emulation would also lead to an enhancement of the authorship effect. In the case of pen traces an extra step is required whereby the consequences of movement (i.e., the pen traces) would need to be mapped onto those effectors that are responsible for producing that movement. Before this step occurs the emulator will not have access to information about which effector's movements to model, and this would reduce the influence that the observer's motor dynamics have on their predictions (the authorship effect). This mapping step may take extra time be- 


\section{Action synchronisation with biological motion}

cause the relevant effectors are not actually present in the stimulus, and these mappings may need to be learnt through the course of the experiment. The presence of this extra step in the experiments by Flach et al. (2003) and the absence of this extra step in the present study may account for our failure to replicate the block effect observed by Flach et al. (2003).

\subsubsection{Conclusions}

Taken together, the results of the present study suggest that observers generate predictions of how other agents will move based on information about how they themselves move. This is consistent with the emulator hypothesis of action prediction whereby observers' action systems form part of a forward model for interpersonal action prediction.

Future studies will need to rule out the possibility that these results can be explained by the coincidence of idiosyncratic tempo, and will need to address the exact role that bodily information might play in action prediction. Furthermore, the exact neural substrate of the emulator has not been investigated. If, as Wilson and Knoblich (2005) suggest, the emulator is instantiated in the human mirror-neuron system then it may be possible to employ the stimuli used in the present study to investigate differences in mirror neuron activity that correlate with prediction accuracy. These stimuli are well suited to this task due to the somatatopic nature of the mirror-neuron system and because these stimuli avoid the block effect observed in previous studies.

\subsection{References}

Buccino, G., Binkofski, F., Fink, G. R., Fadiga, L., Fogassi, L., Gallese, V., ... Freund, H. J. (2001). Action observation activates premotor and parietal areas in a somatotopic manner: an fMRI study. European Journal of Neuroscience, 13(2), 400-404.

Flach, R., Knoblich, G., \& Prinz, W. (2003). Off-line authorship effects in action perception. Brain and Cognition, 53(3), 503-513. 
Fraisse, P. (1982). Rhythm and tempo. In D. Deutsch (Ed.), The psychology of music (pp. 149180). New York: Academic Press.

Gallese, V., \& Goldman, A. (1998). Mirror neurons and the simulation theory of mindreading. Trends in Cognitive Sciences, 2(12), 493-501.

Gallese, V., Fadiga, L., Fogassi, L., \& Rizzolatti, G. (1996). Action recognition in the premotor cortex. Brain, 119(2), 593-609.

Grush, R. (2004). The emulation theory of representation: motor control, imagery, and perception. Behavioral and Brain Sciences, 27(3), 377-396.

Jacob, P. (2008). What do mirror neurons contribute to human social cognition? Mind \& Language, 23(2), 190-223.

Kandel, S., Orliaguet, J. P., \& Viviani, P. (2000). Perceptual anticipation in handwriting: the role of implicit motor competence. Perception \& Psychophysics, 62(4), 706-716.

Knoblich, G., \& Flach, R. (2001). Predicting the effects of actions: interactions of perception and action. Psychological Science, 12(6), 467-472.

Knoblich, G., Seigerschmidt, E., Flach, R., \& Christensen, W. (2002). Authorship effects in the prediction of handwriting strokes: evidence for action simulation during action perception. The Quarterly Journal of Experimental Psychology: Section A, 55(3), 10271046.

Kohler, E., Keysers, C., Umiltà, M. A., Fogassi, L., Gallese, V., \& Rizzolatti, G. (2002). Hearing sounds, understanding actions: action representation in mirror neurons. Science, 297(5582), 846-848.

Miall, R. C., Weir, D., Wolpert, D., \& Stein, J. (1993). Is the cerebellum a smith predictor? Journal of Motor Behavior, 25(3), 203-216.

Oldfield, R. C. (1971). The assessment and analysis of handedness: the edinburgh inventory. Neuropsychologia, 9(1), 97-113.

Repp, B. H. (2005). Sensorimotor synchronization: a review of the tapping literature. Psychonomic Bulletin \& Review, 12(6), 969-992. 
Repp, B. H., \& Keller, P. E. (2008). Sensorimotor synchronization with adaptively timed sequences. Human Movement Science, 27(3), 423-456.

Spray, J. A. (1986). Absolute error revisited: an accuracy indicator in disguise. Journal of Motor Behavior, 18(2), 225-238.

Tesche, C. D., \& Karhu, J. J. T. (2000). Anticipatory cerebellar responses during somatosensory omission in man. Human Brain Mapping, 9(3), 119-142.

Viviani, P., \& Stucchi, N. (1992). Biological movements look uniform: evidence of motorperceptual interactions. Journal of Experimental Psychology: Human Perception and Performance, 18(3), 603-623.

Viviani, P., \& Terzuolo, C. (1982). Trajectory determines movement dynamics. Neuroscience, 7(2), 431-437.

Wilson, M., \& Knoblich, G. (2005). The case for motor involvement in perceiving conspecifics. Psychological Bulletin, 131(3), 460-473.

Wolpert, D. M., Miall, R. C., \& Kawato, M. (1998). Internal models in the cerebellum. Trends in Cognitive Sciences, 2(9), 338-347. 


\title{
CHAPTER 3
}

\section{Motor experience modulates the effect of limb and joint}

\author{
information
}

\subsection{Introduction}

Many types of joint action require two actors to coordinate their actions. Such coordination is especially demanding for joint actions, such as ensemble music and dance performance, where successful completion of the joint action requires precise temporal synchronisation. In these contexts, it is not possible for individuals to observe and then react to the actions of their co-actors because this would introduce disruptive delays. Rather, individuals must anticipate the actions of their co-actors so that they can plan actions that will align with those actions. Because of these time constraints in joint action coordination, researchers have emphasised the role of prediction in recent theoretical accounts of joint action coordination (Csibra, 2008; Vesper, Butterfill, Knoblich, \& Sebanz, 2011; Wilson \& Knoblich, 2005).

Models of predictive mechanisms in motor control, such as forward models (Wilson \& Knoblich, 2005) and inverse models (Csibra, 2008), can greatly inform our understanding 
of joint action coordination (see also Chapter 5). Both classes of models are contained within the model of motor control developed by Wolpert and colleagues (e.g., Wolpert, 1997; Wolpert \& Kawato, 1998). According to this framework, forward and inverse models are used in tandem to achieve goal-directed behaviour when regular feedback is unreliable because of delays or inaccuracies.

Inverse models act as controllers by transforming a goal state into a series of control commands that are then sent to the controlled system (or plant) so that it produces the desired behaviour. In the motor control system, this is implemented by a system that, for example, takes the goal state and transforms it into a series of motor commands that can then be sent to the limb to cause it to move in the desired fashion. Forward models, on the other hand, take the control commands and transform them into a goal state. (Joined together, inverse and forward models perform an identity mapping, transforming goal states into control commands and then back into goal states). The plant (the limb in our motor control example) also performs a forward mapping from control commands into a goal state. Therefore, the forward model can be used to predict how the plant is expected to behave. A forward model is particularly useful in motor control where it can be used to bypass delays that occur because feedback must be transmitted from the periphery to centrally located motor control regions. A forward model can compensate for these delays by substituting predicted feedback for actual feedback from the periphery. In the case of motor control, the forward model is also located centrally and, therefore, this feedback is not subject the same transmission delays.

\subsubsection{Prediction of observed actions within the motor system}

In addition to their role in intrapersonal action prediction, forward models and inverse models are also implicated in the interpersonal action prediction needed for joint action. Csibra (2008) has suggested that during action observation, the mirror-neuron system allows observers to reconstruct the motor codes used to produce the observed action, in a manner analogous to an inverse model. To support this claim, Csibra (2008) cites evidence 
from electrophysiological studies on monkeys as well as neuroimaging studies from humans that show that neurons in motor regions are active not only when actions are produced but also when the same, or similar, actions are passively observed (for a review, see Rizzolatti \& Craighero, 2004). Additionally, Wilson and Knoblich (2005) have proposed that observers are able to construct an internal model of observed actions by mapping the actions onto their own motor systems in a part-by-part, or isomorphic, manner. This internal model acts as a forward model by generating a real-time simulation of the observed action that runs in parallel with incoming sensory information. Information from this model can be substituted for incoming sensory information that reaches the observer through observation. By using internally generated information to drive action planning, delays that result from the processing of external actions can be overcome, and this allows co-actors to plan and coordinate joint actions in time critical situations. Taken together, forward and inverse models provide an effective mechanism that allows actors to use their own action systems in order to emulate ${ }^{1}$ the actions of others. We refer to this proposal as the emulator hypothesis of action prediction.

A key prediction of the emulator hypothesis is that traces of the observer's motor system should be manifested in the predictions that they generate. The authorship effect provides a means of assessing this. The authorship effect refers to the finding that observers are more accurate at generating predictions about recordings of self-generated actions relative to other-generated actions. More generally, the greater the alignment between the motor dynamics of the observer and the motor dynamics of the agent producing the observed action, the more accurate the predictions generated by the observer. The authorship effect has been reported in action-effect prediction tasks where participants are required to synchronise a sensory effect produced by an action, such as a dynamic pen trace that results from writing actions (Flach, Knoblich, \& Prinz, 2003) or about sound patterns that result from key presses during piano playing (Keller, Knoblich, \& Repp, 2007). More

\footnotetext{
${ }^{1}$ Emulator/emulate to refers to a system that duplicates, or the process of duplicating, the functions of a system (e.g., a conspecific's motor system) using different means (e.g., the observer's motor system).
} 
recently, the authorship effect has also been found when observers generate predictions about the unfolding dynamics of an action itself (Chapter 2).

Comparing the data from visual action-effect prediction tasks (Flach et al., 2003) with the data from visual action prediction (Chapter 2) suggests that there may be a performance benefit when observers view the actions directly, rather than just viewing the sensory consequences of the action. Flach et al. (2003) found that the authorship effect took time to emerge, emerging only in the third and fourth (of four) blocks of the experiment. In contrast, in Chapter 2 we reported evidence that the authorship effect emerged immediately, and was unchanged over the course of the experiment. One reason for this discrepancy is that action-effects only contain limited information about the effector used to produce the effect. As a result, participants may need to learn the mapping between the action-effect and the effector used to produce the action before they can construct an isomorphic model. When employing stimuli of actual action, however, the stimuli contain all the information needed to construct an isomorphic model of the action and, therefore, action-effect mappings do not need to belearnt through the course of the experiment. Since the authorship effect is thought to arise from the action of an isomorphic forward model, this difference in learning requirements may explain why the authorship effect emerges at different points in the two paradigms. Consistent with this interpretation, action-effect paradigms that use participants with particularly strong action-effect associations have also been able to eliminate the delay in the emergence of the authorship effect (Keller et al., 2007). Participants with expertise for particular action-effect couplings, such as expert musicians (Bangert et al., 2006; Drost, Rieger, Brass, Gunter, \& Prinz, 2005; Repp \& Knoblich, 2007), may find it easier to map the observed action-effects onto their action repertoire because the action-effects provide sufficient information to determine what actions were employed to produce the stimulus (see also, Schubotz, 2007). 


\subsubsection{Event prediction within the motor system}

In addition to the motor system's role in predicting the actions of others, evidence from paradigms employing abstract stimuli suggest that the motor system might also be used for sequence prediction in general, and not just for observed actions. For example, an fMRI study by Schubotz and von Cramon (2004) employed a sequence prediction task where participants were required to observe a sequence of stimuli and then generate a prediction about the nature of the final stimulus. This task employed both abstract and biological stimuli. The results showed increased BOLD response in overlapping regions in ventral premotor regions during the prediction task for both the abstract stimuli and the biological stimuli. Similarly, Schubotz, Sakreida, Tittgemeyer, and von Cramon (2004) examined the performance of patients with premotor lesions, prefrontal lesions, and matched controls on a sequence prediction task. The task involved viewing sequences of images conforming to a particular pattern and then making a judgement about whether the final stimuli conformed to the pattern. To successfully complete this task participants needed to use the pattern of the sequence to predict the identity of the final stimuli. The results showed that patients with premotor lesions made more errors than controls, and this pattern was not replicated for prefrontal patients relative to controls. Taken together, these data suggest that motor regions - in particular, ventral premotor regions-are involved in sequence prediction in general and not only the prediction of actions.

Schubotz (2007) has suggested that motor simulation is a general mechanism for predicting events. In the case of reproducible events - that is, human actions-it is possible to simulate these events using the same means that were initially employed to create the event by using an internal model of the action. However, this is not possible in the case of irreproducible events, such as abstract stimuli or pattern sequences, which cannot be directly mapped onto the observer's body. In this case, Schubotz (2007) argues that when generating predictions about irreproducible stimuli, or stimuli that cannot be directly mapped onto the observer's body, rather than generating an internal model that is isomorphic with the observed events, observers might rather simulate the observed 


\section{Motor experience modulates limb and joint information}

stimuli using an action model for the effector that best matches the general dynamics of the stimuli. Although it might be possible to model the general dynamics of the stimulus, this model will not provide a complete description of the stimulus dynamics but would rather only provide an approximate description. Similarly, when the stimuli do not contain enough detail about which effectors were used to produce the movement, it might only be possible for observers to map the action onto their body in an approximate manner. Again, this approximate solution might not be able to completely replicate the dynamics of the stimuli-for example, it may not replicate any of the constraints that are specific to particular effectors actually used to create stimuli-and this should be evident as a decrease in prediction performance. Thus, the content or the nature of a stimulus might strongly influence how observers are able to simulate the stimulus within their motor system, and this in turn might affect the accuracy of any predictions generated.

An approximate solution might also be the only solution available in the case of actions that are not part of the observer's repertoire or for actions that observers do not have experience producing. In these cases, observers might again only simulate the general dynamics of the stimulus using the dynamics of an appropriate effector rather than simulating the actual effectors used to perform the action. For example, while a skilled pianist listening to a piece of music might generate predictions about the music by simulating the actual finger movements employed by the performer, in the case of a novice only the general dynamics would be simulated using motor regions that may be unrelated to the production of the action (e.g., vocal or articulatory regions). Indeed, neuroimaging evidence that suggests motor activation during action observation is modulated by motor experience (Calvo-Merino, Glaser, Grézes, Passingham, \& Haggard, 2005; Calvo-Merino, Grézes, Glaser, Passingham, \& Haggard, 2006) supports the idea that experts and naïve observers simulate actions differently. Schubotz (2007) suggests that when observers view actions that are part of their repertoire, the observed actions can be mapped part-by-part onto their motor systems because it already contains an explicit model of the actions generated by performing the action earlier. Thus, both the nature of the stimuli and nature of 
the observer's motor experience might influence the way in which observed actions are mapped onto the observer's motor system. And this should have consequences for the way in which they generate predictions about those actions.

\subsubsection{Aims of the current study}

The primary aim of the present study is to examine the nature of the internal model that observers construct during action prediction. In particular, our aim is to examine whether action prediction is achieved via a general purpose predictive system that, although implemented with the motor system, does not rely on a part-by-part simulation of the observed action but rather just models the general dynamics of the stimuli. The present study measured prediction accuracy by means of an action synchronisation task similar to that reported in Chapter 2; however, rather than examining differences in synchronisation accuracy for self-produced and other-produced actions, all participants viewed other-produced actions and we instead varied the properties of the stimulus as well as the relevant motor experience of the observers. Both manipulations were designed to modify the information that participants could access to allow them to map the observed actions onto their own action systems. The motor experience manipulation was designed to provide observers with an internal representation of the action onto which they could map the stimulus, while the stimulus manipulation was designed to modify whether the stimulus could be directly mapped onto the observers' bodies.

To modify the information content in the stimulus, we constructed two sets of stimuli so that the stimulus either contained information about what effectors were employed to produce the action (full information) or only contained the motion information required to perform the synchronisation task, but excluded any information about the effectors used to produce the action (point information). One might expect that the addition of extra information would always lead to an increase in performance. However, the error in this intuition can be seen when one considers the addition of information that is not relevant to the task. If a general purpose motor mechanism, which only models the dy- 


\section{Motor experience modulates limb and joint information}

namics of observed stimuli without replicating the action, is responsible for action prediction, then providing explicit information about the action should not alter synchronisation performance, as this information will not be incorporated into the predictive model. Only the general dynamics of the stimulus is relevant, and this is held constant across the two stimulus sets. Moreover, the additional information in the stimulus in the full information condition might even serve as a distracter, and this may result in poorer performance. However, if action prediction for biological stimuli relies on constructing a model that replicates the particulars of the effectors used to produce the observed action (an isomorphic model), then providing information about the effectors should enhance synchronisation accuracy as this will assist in constructing a more accurate model. By providing only movement information (point information condition) it will only be possible to replicate the action in an approximate manner. This approximate model would not be capable of fully describing the stimulus, because it would not take into account constraints of the particular configuration of the limb and joints used to produce the action.

A manipulation was also designed to examine the role of motor experience on action emulation. This was achieved by dividing the participants into two groups and only providing one of the groups with experience with actually performing the action that they would later observe. Schubotz (2007) has suggested that when observers predict actions that are part of their action repertoire, they emulate the actions using an internal model of that action that has been laid down by the experience of producing the action. Naïve observers, on the other hand, might only employ motor regions that match the general dynamics of the movement. If this is the case, then we can predict that the effect of stimulus content would be modulated by motor experience. In particular, we can predict that naïve observers would not incorporate information about the effectors used to produce the movement into their predictive model; thus, the addition of this information should not have an effect on their synchronisation performance.

Whether there will be any differences between the two groups (naïve and experienced) in overall synchronisation accuracy is more difficult to predict. One might expect that 
an isomorphic model would be better at predicting the trajectory of a limb's movement as it would capture some of the movement constraints particular to that limb. However, in the present study, participants are always required to view actions performed by other people. Therefore, it may be the case that if the fine details of the action vary between individuals, then including these details in the predictive model might actually lead to poorer performance because it would result in an interference effect. Thus, we can only predict that when participants do employ an isomorphic model, providing more information with which to construct the isomorphic model will result in superior prediction. And, we can further predict that when participants are not employing an isomorphic model, providing additional information with which to construct a better isomorphic model should have no effect. However, it remains unclear whether any overall differences between the groups will emerge.

\subsection{Methods}

\subsubsection{Participants}

The motor experience group consisted of 13 participants ( 11 females), with an average age of 28.1 years (range $=18.8$ to 48.8 years). They were all right-handed (average score 1.82), as assessed by the Edinburgh Handedness Inventory (Oldfield, 1971). The naïve group consisted of 12 participants ( 8 females), with an average age of 20.7 years (range $=18.7$ to 22.8 years). They were all right-handed (average score 1.49). All participants had normal or corrected-to-normal vision and were free of movement difficulties. All procedures were approved by the Macquarie University Human Subjects Ethics committee.

\subsubsection{Stimuli}

\subsubsection{Movement task}

In order to create the stimuli for the test session, five right-handed females with a mean age of 24.8 years (range $=21$ to 31 years) performed the movement task while their move- 

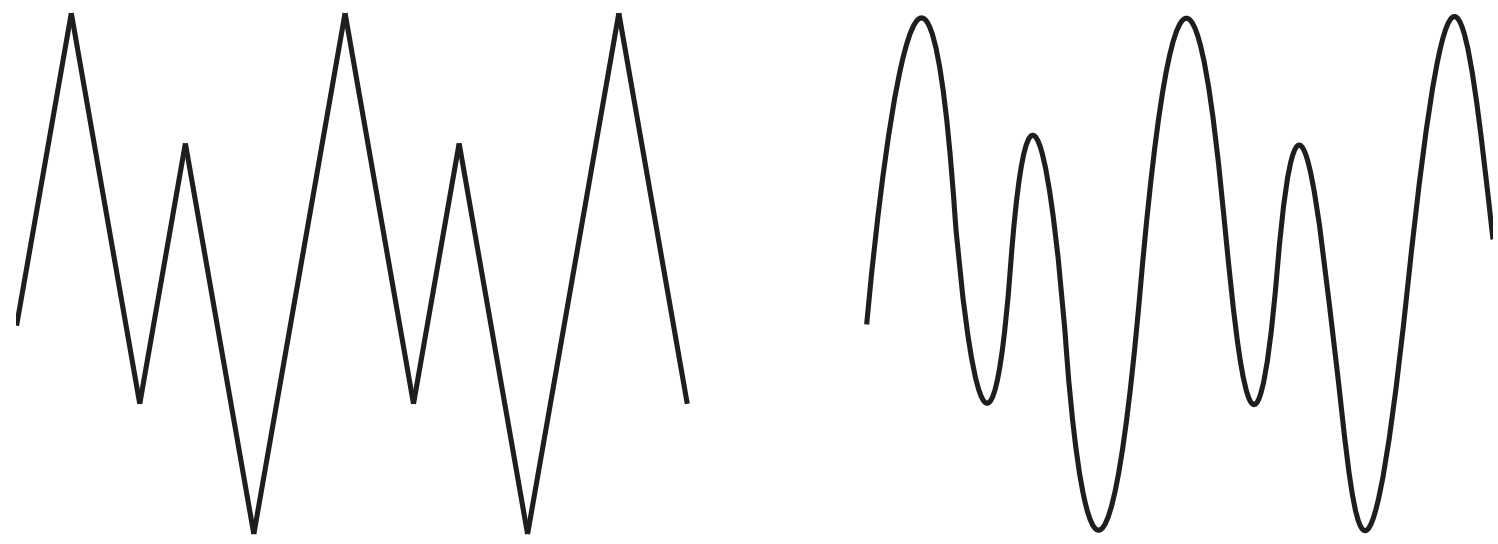

Figure 3.1. The zigzag (left) and wave (right) patterns used as stimuli during the recording session.

ments were tracked with motion capture.

The movement task involved tracing out wave and zigzag patterns (see Figure 3.1) as if drawing them on an imaginary blackboard. The patterns were displayed on two large sheets of cardboard measuring $0.594 \mathrm{~m} \times 0.841 \mathrm{~m}$. Both patterns contained five upward and five downward movements, with the length of the upward movements alternating between long and short. The two patterns differed in terms of the nature of the direction change at the apex of each upward movement. The direction changed sharply for the zigzag pattern, while there was a smooth, flowing direction change for the wave pattern. For the zigzag pattern, the length of the first up-stroke was $0.3 \mathrm{~m}$ with the reaming short up-strokes measuring $0.25 \mathrm{~m}$. The length of the long up-strokes was $0.5 \mathrm{~m}$. The down-strokes were all $0.375 \mathrm{~m}$ in length. The up-strokes and down-strokes met at an angle of 0.35 radians for the zigzag pattern. The stroke lengths for the wave pattern were closely matched to the zigzag and the eccentricity of the curves for the wave pattern was approximately 0.95 .

\subsubsection{Motion capture}

For the purposes of stimulus generation, movements were recorded using an 8-camera 3-D passive optical motion capture system (Vicon MX with 4 Vicon MX-F20 and 4 Vicon 


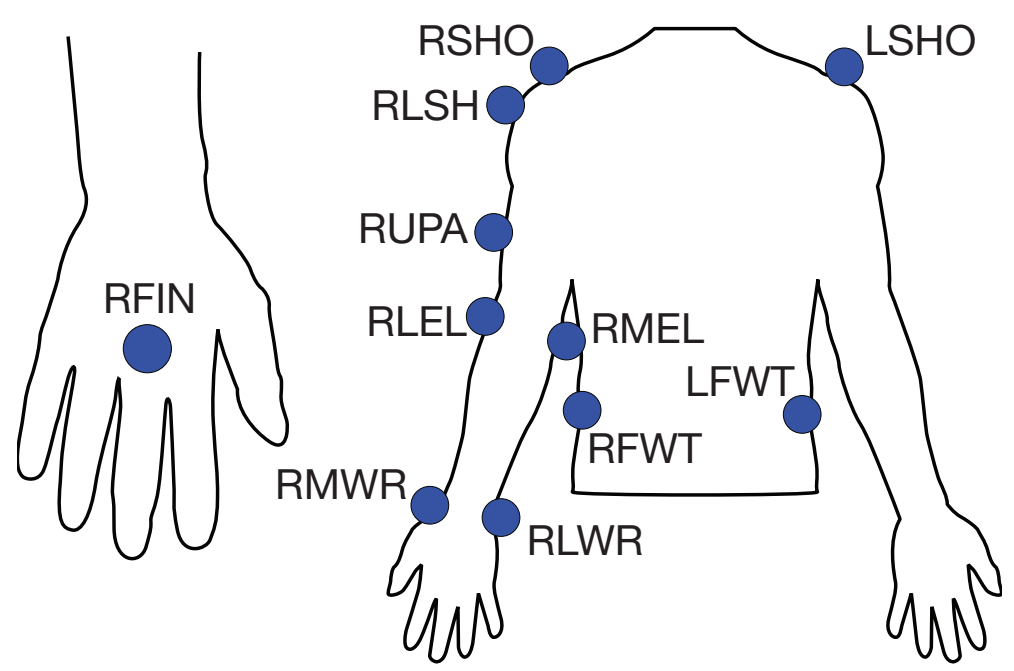

Figure 3.2. Marker positions for the 11 reflective markers used during the recording session.

MX13 + cameras) at a sampling rate of $200 \mathrm{~Hz}$. A total of 11 spherical markers (9 mm in diameter) were placed on the subject's shoulders, right arm, right hand, and waist (see Figure 3.2). Shoulder markers were placed on the top of the left (LSHO) and right shoulder (RSHO), with an additional marker placed on the lateral side of the right shoulder (RLSH). Markers were also placed on the lateral side of the upper arm (RUPA) and on the medial (RMEL) and lateral side (RLEL) of the elbow joint. For the right hand, markers were placed on the lateral (RLWR) and medial (RMWR) side of the wrist, and at the base of the middle finger (RFIN). To define the lower extend of the upper torso, markers were placed on the waist, between the iliac crest and the rib cage, on the left (LFWT) and right (RFWT). One finger maker was used at the base of the right finger (RFIN). To prevent the fingers moving independently from the rest of the hand all movements were performed while participants either held a pen or clenched their fist, whichever was most comfortable.

\subsubsection{Movement description}

All movements used for stimulus creation followed the required big-small-big-small-big pattern of alternating peak heights. For the selected movements, the length of each move- 
ment (total distance travelled by the RFIN marker) was between $2.755 \mathrm{~m}$ and $6.513 \mathrm{~m}$ $(M=4.090 \mathrm{~m}, S D=1.101)$. The average length of each long up-stroke was $0.431 \mathrm{~m}(S D=$ 0.118 ; range $=0.304$ to $0.700 \mathrm{~m}$ ), while the average length of each short up-stroke was $0.328 \mathrm{~m}(S D=0.096$; range $=0.196$ to $0.547 \mathrm{~m})$. This difference was statistically significant $\left(t_{19}=7.815, p<.001\right)$. The average length of the down-strokes was $0.421 \mathrm{~m}$ $(S D=0.112 ;$ range $=0.287$ to $0.664 \mathrm{~m})$. The duration of each movement was on average $7406.25 \mathrm{~ms}(S D=590.23$; range $=6450$ to $8775 \mathrm{~ms})$. The average duration of the upward movements for the long strokes was $721.67 \mathrm{~ms}(S D=64.45$; range $=622$ to $848 \mathrm{~ms})$ and for the short strokes was $649.50 \mathrm{~ms}(S D=69.25 \mathrm{~ms}$; range $=573$ to $855 \mathrm{~ms})$. This difference was statistically significant $\left(t_{19}=7.791, p<.001\right)$. The average duration of the downward movements was $758.20 \mathrm{~ms}(S D=68.01$; range $=640$ to $903 \mathrm{~ms})$. The mean inter-peak duration (the interval between a peak and the preceding peak) was $1413.25 \mathrm{~ms}$ $(S D=108.55 ;$ range $=1195$ to $1665 \mathrm{~ms})$ for the peaks of the long up-strokes, and 1463.63 $\mathrm{ms}(S D=161.62$; range $=1250$ to $1945 \mathrm{~ms})$ for the peaks of the short up-strokes. This difference in duration was also statistically significant $\left(t_{19}=2.143, p=0.045\right)$.

\subsubsection{Test stimuli}

Raw motion capture data was resampled to $25 \mathrm{~Hz}$ and processed with C-Motion Visual 3D (C-Motion INC, Rockville MD) to create the test stimuli. For the full information condition, the motion capture data was rendered as an animated character consisting of an upper torso, right arm and hand (see Figure 3.3 left). The torso was defined by the shoulder markers (RSHO and LSHO) and the waist markers (RFWT and LFWT). The right upper arm extended from the shoulder marker to the elbow markers (RMEL and RLEL) and the lower arm extended from the elbow markers to the wrist markers (RMWR and RLWR). To construct the hand, the wrist markers and the right finger marker (RFIN) were used. For the point information condition, only a single moving point that tracked the movement of the right finger marker was used (see Figure 3.3 right). 

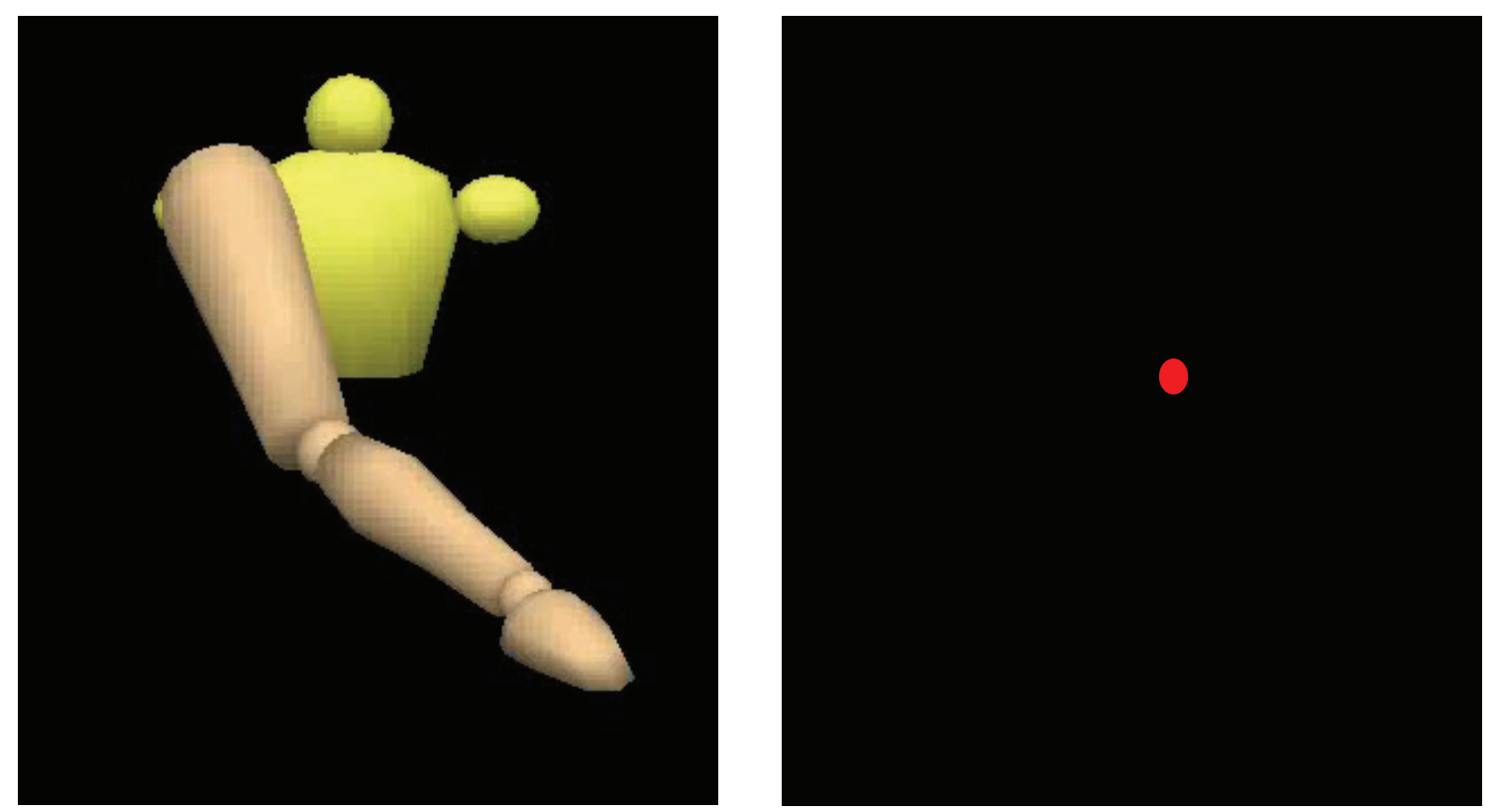

Figure 3.3. Example stimuli from the full information condition (left) and the point information condition (right).

\subsubsection{Procedure}

\subsubsection{Movement session}

Participants in the motor experience group undertook a movement session that was identical to the task employed during stimulus creation (see Section 3.2.2.1). Participants performed 3 blocks containing 5 repetitions of each pattern (in random order) with their eyes closed to limit visual experience. The separation between the movement session and the test session was, on average, 15.85 days (range $=7$ to 27 days).

\subsubsection{Test session}

The task in the test session was to press the response button when the hand of the mannequin, or the marker tracking the hand, reached the apex of each upward movement. Participants were instructed to synchronise the button-press with the display as accurately as possible and were told that this may require them to anticipate when the peak will occur. Each participant performed 4 blocks containing 40 unique stimuli (composed of 20 trials for the full information condition and 20 trials for the point information con- 


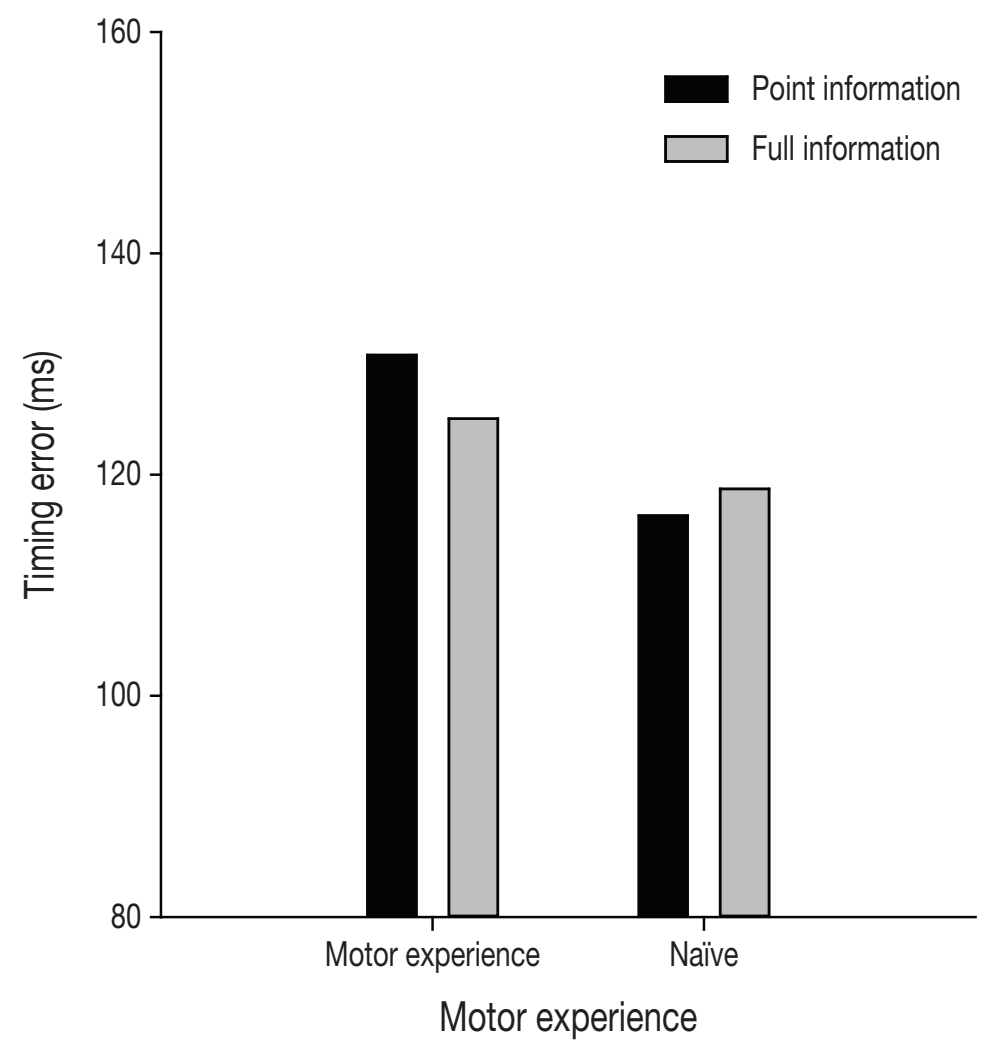

Figure 3.4. Absolute timing error for the full information condition and point information condition for the motor experience group and the naïve group.

dition) with equal numbers of wave and zigzag stimuli. Participants that did not undergo the movement session were given a brief verbal description of the movement task.

\subsection{Results}

Timing error was calculated as the absolute difference between the timing of the peak in the motion capture trajectory and the timing of the button-press. Absolute timing error was used as a dependent measure because it has been shown to provide a good index of accuracy of hitting the target (Spray, 1986). To ensure that participants were attempting to predict the movement of the display, rather than merely observing it and then responding, button-presses with a timing error greater than $600 \mathrm{~ms}$ were dropped from the analyses. This resulted in less than $1 \%$ of button-presses being excluded (including all buttonpresses did not change the overall findings). Absolute timing error was analysed by means 
of a $2 \times 4 \times 2$ mixed ANOVA with the within-subjects factors Information Content (full information, point information) and Block (1, 2, 3, and 4), and the between-subjects factor of Experience (motor experience, naïve). The Greenhouse-Geisser procedure was used to correct for violations of sphericity, where appropriate (we report uncorrected $d f$ s along with the $\epsilon$ value).

There were no systematic differences in synchronisation accuracy related to experimental block, as indicated by the non-significant main effect for Block $\left(F_{3,69}=0.028, p=.941\right.$, $\epsilon=.503, \eta_{p}^{2}=.001$ ), and the non-significant interactions for Information Content $\times$ Block $\left(F_{3,69}=0.854, p=.469, \eta_{p}^{2}=.036\right)$, Block $\times$ Experience $\left(F_{3,69}=0.455, p=.584, \epsilon=.503\right.$, $\left.\eta_{p}^{2}=.019\right)$ and Information Content $\times$ Block $\times$ Experience $\left(F_{3,69}=0.183, p=.907, \eta_{p}^{2}=.008\right)$. There were also no systematic differences in synchronisation accuracy between the naïve group and the group with motor experience, as indicated by the non-significant main effect of Experience $\left(F_{1,23}=0.407, p=.530, \eta_{p}^{2}=.017\right)$. Furthermore, there were no systematic differences in synchronisation accuracy between the full information displays and point information displays when the data were collapsed across group and block, as indicated by the non-significant main effect for Information Content $\left(F_{1,23}=1.400, p=.249\right.$, $\left.\eta_{p}^{2}=.057\right)$

As predicted, the results showed that the effect of information content was modulated by motor experience, as indicated by the significant interaction for Information Content $\times$ Experience $\left(F_{1,23}=8.243, p=.009, \eta_{p}^{2}=.264\right)$. To decompose this interaction, the data were collapsed across block and two paired t-tests were conducted to examine the difference between the two levels of Information Content (full information, point information) for each Experience group. The results of these t-tests showed that the information content effect (full information compared with point information) was found only for the motor experience group $\left(t_{12}=2.871, p=0.014\right)$ but not the naïve group $\left(t_{11}=-1.196\right.$, $p=.257)$. This indicates that timing error was significantly higher for the point stimuli relative to full stimuli for the motor experience group $\left(M_{\text {diff }}=5.76,95 \% \mathrm{CI}=1.39\right.$ to 10.13$)$ but not for the naïve group $\left(M_{\text {diff }}=-2.40,95 \% C I=-6.60\right.$ to 1.81$)$. This suggests that only 
Peak timing and button-presses

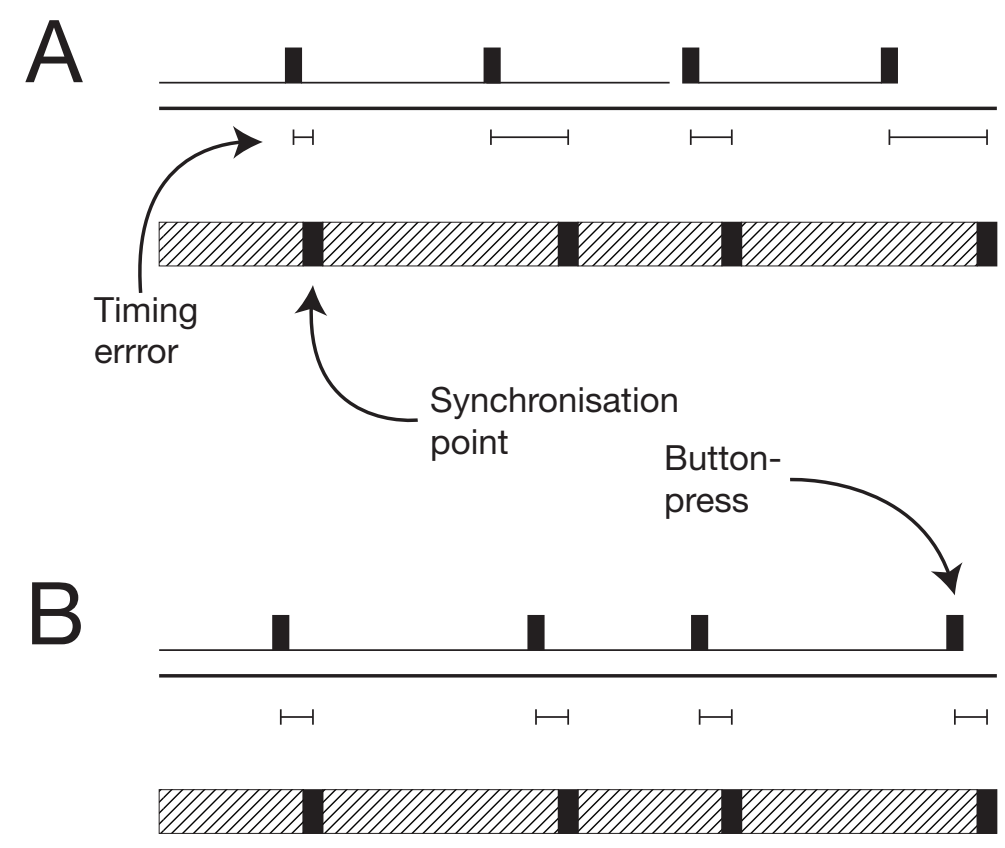

Sample data
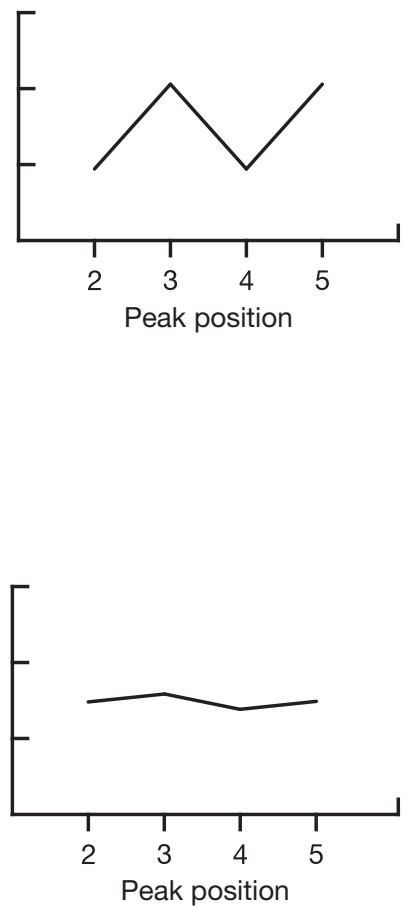

Figure 3.5. (A) Evenly spaced button-presses results in timing errors that vary as a function of peak number. (B) Timing error that does not vary as a function of peak number is a result of the timing of button-presses varying as a function of peak number.

the motor experience group was able to take advantage of the presence of limb and joint information to enhance synchronisation accuracy. These data are displayed in Figure 3.4.

In addition to the motor experience group's ability to exploit limb and joint information for enhanced prediction accuracy, a further attempt was made to quantify the difference in task performance between the motor experience group and the naïve group. Following the logic of Flach et al. (2003), we examined whether there were differences in task performance that related to whether participants primarily responded to local aspects or global aspects of the stimulus. In the visual stimuli presented to participants the height and, therefore, the duration of each upward movement alternated from long to short. Additionally, the duration between consecutive peaks alternated between long and short (see Section 3.2.2.3). This irregular pattern leads to local variations in peak timing. If participants based their responses on global aspects of the stimuli, such as average 


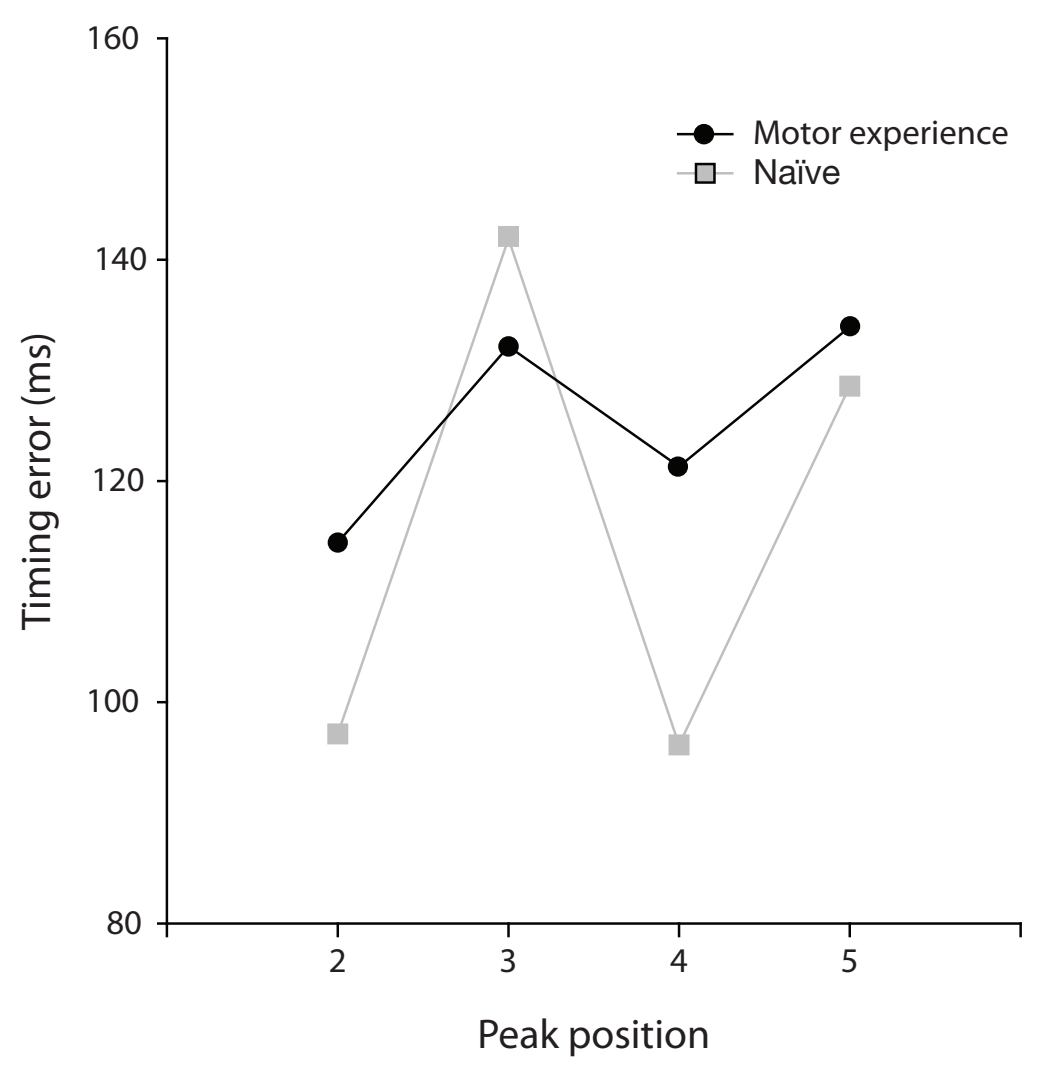

Figure 3.6. Timing error as a function of peak position for the motor experience group and the naïve group.

tempo, a characteristic pattern of timing errors would emerge with timing error fluctuating from peak to peak (see Figure 3.5A). If, on the other hand, participants adjusted their responses according to the local variations in the stimuli then the peak-to-peak fluctuations should decrease in amplitude with timing error being approximately equal for each of the button-presses (see Figure 3.5B). Data was first collapsed across Information Content and Block, and then Timing error for each of the final four button-presses (corresponding to each of the final four peaks) was analysed by means of a $4 \times 2$ mixed ANOVA with the within-subjects factor of Peak Position and the between-subjects factor of Experience, and planned comparisons were conducted on each group using a one-way ANOVA. (The first peak was dropped from the analysis because the movement leading up to the first peak is neither clearly long nor short). If the individual peak means fluctuate as illustrated in Figure 3.5A, then this should be evident as a significant cubic trend.

The analysis yielded a significant cubic tend for Peak Position $\left(F_{1,23}=17.671, p<.001\right.$, 
$\left.\eta_{p}^{2}=.529\right)$ and a Peak Position $\times$ Experience interaction that approached significance $\left(F_{1,23}=4.047, p=.056, \eta_{p}^{2}=.150\right)$. Planned comparisons, conducted on each group individually, showed that the cubic trend for Peak Position was significant only for the naïve group $\left(F_{1,11}=12.621, p=.003, \eta_{p}^{2}=.560\right)$, and not for the motor experience group $\left(F_{1,12}=3.573, p=.083, \eta_{p}^{2}=.229\right)$. This suggests that for the naïve group timing error changed in a low-high-low-high pattern as the trial progressed, while for the motor experience group peak position did not significantly affect timing error (see Figure 3.6). These results are consistent with the naïve group responding to global aspects of the stimuli and the motor experience group responding to local aspects of the stimuli.

\subsection{Discussion}

The primary aim of the present study was to investigate the nature of the action emulation employed during action prediction. In particular, we wanted to investigate whether action prediction relies on observers mapping the stimulus onto their body in a part-bypart manner, or whether they just model the general dynamics of the action without modelling the specifics of the effectors used to create the stimulus. Furthermore, our aim was to investigate the influence of motor experience on action emulation. Schubotz (2007) has suggested that while abstract stimuli and actions that are not part of the observer's repertoire might be simulated using a general purpose mechanism, actions an observer has experience producing are instead simulated using a model that incorporates the specifics of the effectors used to produce the movement. In order to examine these questions, we varied both the information content of the stimuli (employing stimuli that both did and did not contain information about the effector used to produce the movement) and we varied the motor experience of the observers (they either had experience producing the action or had no experience producing the action). 


\subsubsection{The effect of stimulus information}

In the full information condition, the visual stimulus contained not only the movement information required to perform the task, but also information about the effector used to produce the movement (in this case, the right hand and arm) and the configuration of the limb and the joints of that effector during action production. In the point information condition, the visual stimulus only contained a single moving point. While the point information condition also contained all the movement information required to perform the task, it lacked the additional information about the state of the effectors. As predicted, the results showed enhanced prediction accuracy (measured as absolute timing error on the synchronisation task) when observing the full information stimuli.

An alternative explanation for these results is that a difference in the low-level visual features of the stimuli might account for the differences in prediction accuracy. For example, it might be the case that the point information stimulus, which overall contains less visual information, is harder to visually track, and this may manifest as decreased prediction accuracy. Similarly, the smaller size of the object in the point information stimuli may make it harder to accurately judge the velocity of the movement relative to the larger more complex objects in the full information stimuli.

However, there is a good reason to reject these alternate explanations. If the low-level visual features could account for the differences in synchronisation accuracy between the full information stimuli and the point information stimuli, then this difference should be present in both the experienced and the naïve groups. This was not the case, as the effect of information content was limited to the group with motor experience-the naïve group showed no significant difference between the two conditions. Therefore, the results are not consistent with an explanation based on low-level visual features. Furthermore, the finding that motor experience modulated the effect of stimulus information suggests that the stimulus information effect has a source within the motor system, and is not a perceptual effect.

The finding that limb and joint information was able to enhance synchronisation accu- 


\section{Motor experience modulates limb and joint information}

racy is also consistent with what is known about the mirror-neuron system, the putative substrate of the action prediction system (Csibra, 2008; Wilson \& Knoblich, 2005). Studies by Buccino and colleagues (Buccino et al., 2001; Buccino et al., 2004) have shown that during action observation, regions of the motor cortex are activated in a somatatopic fashion. That is, certain regions show specificity for particular effectors in a manner similar to Penfield's (1954) motor homunculus.

\subsubsection{The effect of motor experience}

A secondary aim of the present study was to examine what influence motor experience would have on prediction accuracy. The results show that motor experience modulated the effect that stimulus type had on prediction performance. In addition, motor experience had an effect on how participants performed the task. In particular, these data show that while the timing error for experienced participants was not affected by the serial position of the peak, the timing error for naïve participants varied according to peak number, and the timing error for the small peaks was significantly different to the timing error for large peaks.

A pattern of fluctuations would arise if participants kept a relatively steady pace throughout the trial because the spacing of the peaks was not constant throughout the trial, but instead changed according to the height of the peaks. A relatively steady pace for button-presses might occur if participants responded to the global properties of the stimuli, such as the average rhythm (that is, the pace of movement production), or to the general stimulus dynamics. In order to maintain a relatively constant timing error, as seen in the experienced group, participants would need to adjust the timing of each button-press according to the local timing variations in the stimuli that result from the alternating heights of the peaks. This pattern of data, therefore, suggests a global/local bias in stimulus processing that is modulated by motor experience.

The effect of motor experience on processing visual stimuli has recently been noted in several studies. For example, Casile and Giese (2006) have shown that motor training 
enhances a participant's ability to make a fine-grained visual discrimination of action. In their study, participants were asked to make same/different judgements about gait patterns that they either did or did not have motor experience with. In normal walking, people show a $180^{\circ}$ phase difference between opposite arms and legs. In addition to normal walking, Casile and Giese (2006) manipulated point-light walkers so that they showed a $225^{\circ}$ and $270^{\circ}$ phase difference between opposite limbs. From these three prototypes $\left(180^{\circ}, 225^{\circ}\right.$, and $\left.270^{\circ}\right)$ an additional set of stimuli was created by adding further phase differences that ranged from $-45^{\circ}$ to $45^{\circ}$ (in $15^{\circ}$ increments). Participants were asked to sequentially view two different point-light walkers and then decided whether the two walkers had the same gait pattern. After the initial testing session, participants underwent a training session where they were trained to walk with the unnatural $270^{\circ}$ phase relationship (between their arms only). Importantly, participants were blindfolded during the training session, so that they only received motor experience with the action and not visual experience. Following training, the same/different task was then repeated and performance before and after the training was compared. The results showed a significant improvement on the same/different task for the trained $270^{\circ}$ gait pattern, while performance on the unfamiliar $225^{\circ}$ gait pattern was unchanged. The amount by which a participant improved on the discrimination task was also found to correlate with how well that participant had learnt to perform the $270^{\circ}$ phase pattern. Importantly, the improvement in discrimination could not be elicited by providing participants with purely visual training on the unfamiliar gait patterns; only the motor training was effective. These results are consistent with the notion that in order to make fine-grained visual judgements about the kinematics of an action, observers need to have an internalised model of the action, which can be gained through motor experience.

Similarly, Calvo-Merino, Ehrenberg, Leung, and Haggard (2010) tested the performance of female and male dancers as well as non-dancers on a visual discrimination task. The task consisted of judging when two exemplars of a particular female-specific dance move were the same or different. As with Casile and Giese (2006), female dancers, who 


\section{Motor experience modulates limb and joint information}

had motor experience with the dance moves, performed better than male dancers, who only had visual experience with the dance moves. Overall, however, dancers were better than non-dancers suggesting that visual experience can also increase sensitivity to fine kinematic variations, but not as effectively as motor experience.

Our findings build on these earlier results, and suggest that observers with motor experience for the observed action are also better able to make fine-grained predictions about the dynamics of a stimulus. This result is consistent with the notion that experienced observers generate predictions about observed actions by employing an internal model of that action that is acquired through motor experience. By mapping the observed action onto their internal model for that action they are better able to capture the fine-grained timing variations in the stimulus because their predictive model more completely captures the constraints specific to the effectors used to produce the action.

\subsubsection{The relationship between stimulus content and motor experience}

We have argued that participants with motor experience are more sensitive to the finegrained timing differences present in an action because they, unlike naïve observers, employ an internalised model of the observed action in order to generate their predictions. This might also help to explain why the influence of limb and joint information was restricted to the group with motor experience. Several neuroimaging studies have shown experience-related differences in motor system activation when observers view actions performed by other people. For example, Calvo-Merino et al. (2005) asked expert dancers and non-dancer controls to view videos of dancers performing in one of two styles (ballet or capoeira). The results showed that activation in motor regions was greater when dancers viewed performers of their own style, suggesting that the motor system is preferentially engaged when observing actions that are familiar. A follow-up study (CalvoMerino et al., 2006), using male and female ballet dancers extended this finding by showing that motor regions were preferentially activated when viewing gender-specific dance moves. As both male and female dancers presumably have equal visual experience with 
opposite gender dance moves, but different motor experience, this finding suggests that motor engagement with visually presented stimuli is selective for actions for which observers have specific motor familiarity over and above the effects of visual familiarity.

Studies by Schubotz and colleagues (Schubotz et al., 2004; Schubotz \& von Cramon, 2004) have also implicated premotor regions in prediction of abstract stimuli and in sequence prediction in general. These stimuli cannot be mapped onto the observer's body and, therefore, they might rather be predicted by using a predictive model that exploits the dynamics of an effector that most closely matches the dynamics of the stimulus. Similarly, differences in motor system activation related to motor experience might suggest that inexperienced observers employ general predictive mechanisms, such as those used for sequence prediction, even when the observed action can, at least in principle, be mapped onto their body in an isomorphic, or part-by-part, manner. The addition of limb and joint information was designed to assist the process of mapping the observed action onto the observer's body; however, if, as the neuroimaging data cited above suggests, naïve observers less readily map the observed action onto their body in an isomorphic manner, then providing information to assist this process should provide no additional benefit. This is indeed what was found in the present study.

\subsubsection{Conclusions}

Providing observers with information about the configuration of the effectors used to produce an action enhanced their ability to generate real-time predictions about the dynamics of those actions. This enhancement cannot be explained by perceptual differences, as the effect was restricted to observers who had motor experience with the actions. Motor experience also modulated the way in which observers responded to stimuli: Observers with motor experience were more sensitive to local timing variations in the action than observers without motor experience. These findings suggest that naïve participants and experienced participants engage in a different type of motor emulation during action observation. The results provide further support for the theory that action prediction relies 
on motor emulation of observed actions.

\subsection{References}

Bangert, M., Peschel, T., Schlaug, G., Rotte, M., Drescher, D., Hinrichs, H., . . Altenmüller, E. (2006). Shared networks for auditory and motor processing in professional pianists: evidence from fMRI conjunction. NeuroImage, 30(3), 917-926.

Buccino, G., Binkofski, F., Fink, G. R., Fadiga, L., Fogassi, L., Gallese, V., ... Freund, H. J. (2001). Action observation activates premotor and parietal areas in a somatotopic manner: an fMRI study. European Journal of Neuroscience, 13(2), 400-404.

Buccino, G., Lui, F., Canessa, N., Patteri, I., Lagravinese, G., Benuzzi, F., ... Rizzolatti, G. (2004). Neural circuits involved in the recognition of actions performed by nonconspecifics: an fMRI study. Journal of Cognitive Neuroscience, 16(1), 114-126.

Calvo-Merino, B., Glaser, D. E., Grézes, J., Passingham, R. E., \& Haggard, P. (2005). Action observation and acquired motor skills: an fMRI study with expert dancers. Cerebral Cortex, 15(8), 1243-1249.

Calvo-Merino, B., Grézes, J., Glaser, D., Passingham, R., \& Haggard, P. (2006). Seeing or doing? influence of visual and motor familiarity in action observation. Current Biology, 16(19), 1905-1910.

Calvo-Merino, B., Ehrenberg, S., Leung, D., \& Haggard, P. (2010). Experts see it all: configural effects in action observation. Psychological Research PRPF, 74, 400-406.

Casile, A., \& Giese, M. A. (2006). Nonvisual motor training influences biological motion perception. Current Biology, 16(1), 69-74.

Csibra, G. (2008). Action mirroring and action understanding: an alternative account. In P. Haggard, Y. Rossetti \& M. Kawato (Eds.), Sensorimotor foundations of higher cognition (pp. 435-459). New York: Oxford University Press.

Drost, U. C., Rieger, M., Brass, M., Gunter, T. C., \& Prinz, W. (2005). Action-effect coupling in pianists. Psychological Research, 69(4), 233-241. 
Flach, R., Knoblich, G., \& Prinz, W. (2003). Off-line authorship effects in action perception. Brain and Cognition, 53(3), 503-513.

Keller, P. E., Knoblich, G., \& Repp, B. H. (2007). Pianists duet better when they play with themselves: on the possible role of action simulation in synchronization. Consciousness and Cognition, 16(1), 102-111.

Oldfield, R. C. (1971). The assessment and analysis of handedness: the edinburgh inventory. Neuropsychologia, 9(1), 97-113.

Penfield, W. (1954). Mechanisms of voluntary movement. Brain, 77(1), 1-17.

Repp, B. H., \& Knoblich, G. (2007). Action can affect auditory perception. Psychological Science, 18(1), 6-7.

Rizzolatti, G., \& Craighero, L. (2004). The mirror-neuron system. Annual Review of Neuroscience, 27(1), 169-192.

Schubotz, R. I. (2007). Prediction of external events with our motor system: towards a new framework. Trends in Cognitive Sciences, 11(5), 211-218.

Schubotz, R. I., \& von Cramon, D. Y. (2004). Sequences of abstract nonbiological stimuli share ventral premotor cortex with action observation and imagery. The Journal of Neuroscience, 24(24), 5467-5474.

Schubotz, R. I., Sakreida, K., Tittgemeyer, M., \& von Cramon, D. Y. (2004). Motor areas beyond motor performance: deficits in serial prediction following ventrolateral premotor lesions. Neuropsychology, 18(4), 638-645.

Spray, J. A. (1986). Absolute error revisited: an accuracy indicator in disguise. Journal of Motor Behavior, 18(2), 225-238.

Vesper, C., Butterfill, S., Knoblich, G., \& Sebanz, N. (2011). A minimal architecture for joint action. Neural Networks.

Wilson, M., \& Knoblich, G. (2005). The case for motor involvement in perceiving conspecifics. Psychological Bulletin, 131(3), 460-473.

Wolpert, D. M. (1997). Computational approaches to motor control. Trends in Cognitive Sciences, 1(6), 209-216. 
3. Motor experience modulates limb and joint information

Wolpert, D. M., \& Kawato, M. (1998). Multiple paired forward and inverse models for motor control. Neural Networks, 11(7-8), 1317-1329. 
CHAPTER 4

\section{The effect of tempo and dynamic cues on action synchronisation}

\subsection{Introduction}

Recent research in motor control (Kawato, 1999; Wolpert, 1997) has borrowed several key concepts from a branch of engineering known as control theory (for an introduction to control theory, see Golnaraghi \& Kuo, 2010). Two concepts, inverse models (or controllers) and forward models (or predictors), have been especially useful, and they lie at the heart of an influential model of motor control developed by Wolpert (1997). In a control system, the target system-for example, a limb in the motor control system-transforms a control command into the desired behaviour, or goal. In feedback control, the output of the target system can be monitored and compared with the goal behaviour and any deviance in the output can be corrected by adjusting the control commands. In order to compute the control commands, an inverse model is employed. The inverse model is a model of the inverse dynamics of the target system - that is, it models the mapping from goals to commands, rather than from commands to goals. In motor control, the system is also equipped with forward models, which model the forward dynamics of the target 


\section{The effect of tempo and dynamic cues}

system - from control commands to goals. Forward models are also known as emulators because they duplicate the functions of one system (e.g., a limb) using different means (e.g., an internal model).

Forward models are especially useful in motor control because special challenges exist that make it difficult to implement regular feedback control. One such challenge is that feedback cannot be returned to the system controller in a timely fashion. When an action is performed, and a limb begins to move, feedback about the limb's progress has to travel from the periphery to centrally located motor regions. This process takes time and, therefore, when feedback does arrive it may no longer accurately reflect the distal state of affairs. One way to overcome this challenge is to use a forward model to generate a prediction about what feedback is likely to arise. By replacing regular feedback with predicted feedback these delays can be bypassed. Forward models can also be used for calculating state variables (such as the current state of a limb) and for performing sensory cancellation. To compute state variables, the motor control system can, for example, combine information about the predicted location of a limb with whatever sensory information it has about its location yielding a more accurate location estimate than either source of information can provide alone. Moreover, by subtracting or cancelling out the predicted sensory effects of limb movement from the incoming sensory stream, the sensory effects that arise from movements of the limb and movement in the environment can be separated.

Interpersonal action prediction (or simply, action prediction) deals not with how individuals generate predictions about their own actions, as in motor control, but rather how they generate predictions about the actions of conspecifics. Action prediction may play an important role in joint action because it allows actors to anticipate the actions of their co-actors. This enables them to coordinate their actions with the actions of their coactors in a timely fashion. Two recent accounts of action prediction (Csibra, 2008; Wilson \& Knoblich, 2005) have suggested that, rather than action prediction being performed by a purely perceptual system, action prediction relies crucially on the observer's motor sys- 
tem. These accounts have used inverse models (Csibra, 2008) and forward models (Wilson \& Knoblich, 2005) to explain how this might be done. According to these accounts, an inverse model allows an observer to transform the observed action into a motor command that can then be run through the forward model to generate a prediction. Evidence from monkey electrophysiology and human neuroimaging studies suggests that cortical motor regions are active not only during action production but also during passive observation of actions (for a review, see Rizzolatti \& Craighero, 2004). These regions form part of the mirror-neuron system, so called because they allow an observer to "mirror" the observed action with their motor system. Csibra (2008) suggests that these regions perform a function analogous to an inverse model by transforming the goal or intention (of an observed agent) into the motor actions required to realise that goal. The motor code reconstruction is not performed solely on the basis of intentions, but is also facilitated by a process of visual analysis and decomposition of the observed action. While Csibra's (2008) account focuses on the process of reconstructing the motor codes, Wilson and Knoblich (2005) provide an account of how a prediction might be generated. According to their account, the mirror-neuron system also functions as a forward model, or predictor. Once observers have reconstructed the motor commands for the observed actions, these commands can be fed through the observer's forward model for their own actions to generate predictions about the unfolding dynamics of the observed action or the sensory effects that those actions will produce. Taken together, reconstructing the motor codes driving an action (inverse models) and predicting the unfolding dynamics or sensory consequences of an action (forward models) constitute a system that allows observers to duplicate the actions of conspecifics within their own motor system. That is, they constitute a system that allows observers to emulate the actions of conspecifics. This we refer to as the emulator hypothesis of action prediction.

Support for the emulator hypothesis of action prediction has come from several experiments that have employed visual-motor (Flach, Knoblich, \& Prinz, 2003; Chapter 2) and audio-motor (Keller, Knoblich, \& Repp, 2007) self/other prediction paradigms. In these 


\section{The effect of tempo and dynamic cues}

studies, participants are required to observe or listen to recordings of self-produced or other-produced actions while generating predictions about the timing of particular synchronisation points. The logic of this paradigm is that if the action prediction mechanism is implemented in the motor system then, rather than prediction accuracy being purely a function of the stimulus, it should vary according to the match between the stimulus and the observer's motor system. That is, prediction should be more accurate when there is a better match between the action dynamics of the observer and the dynamics of the observed action. When generating predictions about self-produced actions, or actioneffects, the same system that was initially used for producing the action is then later used for prediction. This results in a (in principle) perfect match of system dynamics. An alternative account might be that action prediction is instantiated within a purely perceptual system, with no input from the observer's motor system. In that case, the similarity between the observer's movement dynamics and the observed agent's movement dynamics should not affect prediction accuracy.

Within the visual domain, Flach et al. (2003) compared accuracy on a synchronisation task where the stimuli consisted of dynamic pen traces that had been produced by participants drawing several exemplars of rhythmic wave and zigzag patterns (for example, see Figure 4.1). In their study, the synchronisation points were defined as the apex of each upward movement, and participants were required to generate predictions about the timing of the synchronisation points by making a button-press response. As predicted by the emulator hypothesis, prediction accuracy was better when making predictions about selfproduced actions relative to other-produced actions; this self-prediction advantage was termed the authorship effect. Similar effects have also been reported in Chapter 2 where participants were presented with rhythmic wave and zigzag arm movements. In addition to these experiments in the visual domain, Keller et al. (2007) used the self/other prediction paradigm to examine prediction accuracy in the auditory domain by asking participants to play a piano duet with either a recording of themselves or a recording of another person. As predicted by the emulator hypothesis, synchronisation was better when pianists played duets with recordings of themselves. 
While these results have been used as evidence for action emulation in action prediction, there is an alternative explanation. Previous work has shown that people have a preferred tempo for spontaneous production of rhythmic movements (see Fraisse, 1982, for a review). For example, when people are asked to produce rhythmic movements, such as clapping or finger tapping, they have a preferred idiosyncratic tempo for producing these actions. The existence of a preferred tempo raises the possibility that the authorship effect might arise not from differences in prediction ability for self-produced and other-produced actions, but because people find it easier to synchronise with movements performed at their preferred tempo. That is, the authorship effect might merely be the result of self-similarity in action production.

To investigate the influence of self-similarity, Keller et al. (2007) asked each participant to perform the primo and secondo parts of the duet unaccompanied. These recordings were then compared to examine the similarity in the timing of the events in each part. It was found that the primo and the secondo were no more similar when performed by the same participant than they were when performed by different participants. That is, an authorship effect could not be observed by merely matching two unaccompanied recordings. This suggests that a process over and above self-similarity is responsible for the authorship effect-a process that is active during accompanied performance, such as action emulation. However, it is difficult to say whether the results of Keller et al. (2007) can be generalised to experiments the of Flach et al. (2003) or to the experiment reported in Chapter 2. For example, it is not known whether the results obtained using an auditorymotor prediction paradigm would also apply to visual-motor prediction or whether the results obtained for complex actions, such as piano playing, are applicable to the relatively simple rhythmic actions employed by Flach et al. (2003) and in Chapter 2. Therefore, because neither Flach et al. (2003) nor the experiment presented in Chapter 2 controlled for production tempo, it is not possible to rule out the possibility that the authorship effect reported in these studies was the result of a match between the preferred tempo for producing button-presses and the temporal properties of the stimulus. 


\subsubsection{Aims of the present study}

The aim of the present study is to further explore the mechanisms that underlie action prediction and to examine the possibility that the authorship effect arises due to a match between the temporal properties of the stimulus and the preferred response tempo of the participant. To do this, we examined whether tempo was the primary determinant of synchronisation accuracy-that is, whether synchronisation accuracy was equal for tempomatched stimuli. In order to create the stimuli for the synchronisation task, we employed the same procedure described in Chapter 2. A group of participants, who only took part in the movement task, were asked to perform at series of up-and-down rhythmic arm movements tracing out the shape of a zigzag or wave while motion capture data was recorded. The recorded data was duplicated to create two sets of rhythmically identical stimuli. For one set stimuli, the dynamics between the synchronisation points was altered. The trajectories were modified so that rather than the movements exhibiting a typical natural velocity profile-for example, as described by the two thirds power law (Viviani \& Stucchi, 1992) or minimum jerk model (Flash \& Hogan, 1985) — the movements exhibited a constant velocity profile. If prediction accuracy is dependent on the timing of the synchronisation points, then we should expect to find no differences in synchronisation accuracy for stimuli that have identical timing properties. However, if other factors-such as whether the stimulus dynamics between the synchronisation points resemble the observer's action dynamics - determine synchronisation accuracy, then we should expect to find superior synchronisation accuracy for the stimuli that match human dynamics. 


\subsection{Experiment 1}

\subsubsection{Methods}

\subsubsection{Participants}

Eighteen undergraduate psychology students (14 females) volunteered for the experiment. All were right handed $(M=1.67$; range $=1.11$ to 2$)$, as measured by the Edinburgh handedness inventory (Oldfield, 1971). The participants had an average age of 24 years (range $=18$ to 46 years), were free from movement difficulties, and had normal or corrected-to-normal vision. All procedures were approved by the Macquarie University Human Subject Ethics committee.

\subsubsection{Movement task}

To create the stimuli for the test session five individuals performed a movement task while their movements were recorded using motion capture. All of the participants used for the stimulus creation were female and right handed $(M=1.9$; range $=1.6$ to 2$)$. They had an average age of 24.8 years (range $=21$ to 31 years), were free from movement difficulties, and had normal or corrected-to-normal vision. All procedures were approved by the Macquarie University Human Subject Ethics committee, and all participants consented to their movements being used to create stimuli for experiments.

The movement task involved tracing out wave and zigzag patterns (see Figure 4.1) as if drawing them on an imaginary blackboard. The patterns were displayed on two large sheets of cardboard measuring $0.594 \mathrm{~m} \times 0.841 \mathrm{~m}$. Both patterns consisted of five peaks that alternated in height. For the zigzag pattern, the up-strokes measured $0.25 \mathrm{~m}$ for the small peaks and $0.5 \mathrm{~m}$ for the large peaks (except for the first up-stroke that measured $0.3 \mathrm{~m}$ ). All down-strokes measured $0.375 \mathrm{~m}$. All strokes met at an angle measuring 0.35 radians. The stroke lengths for the wave pattern were closely matched to those of the zigzag pattern, with the eccentricity of the curves measuring approximately 0.95 for all peaks. Participants performed two practice trials with their eyes open to get a sense 

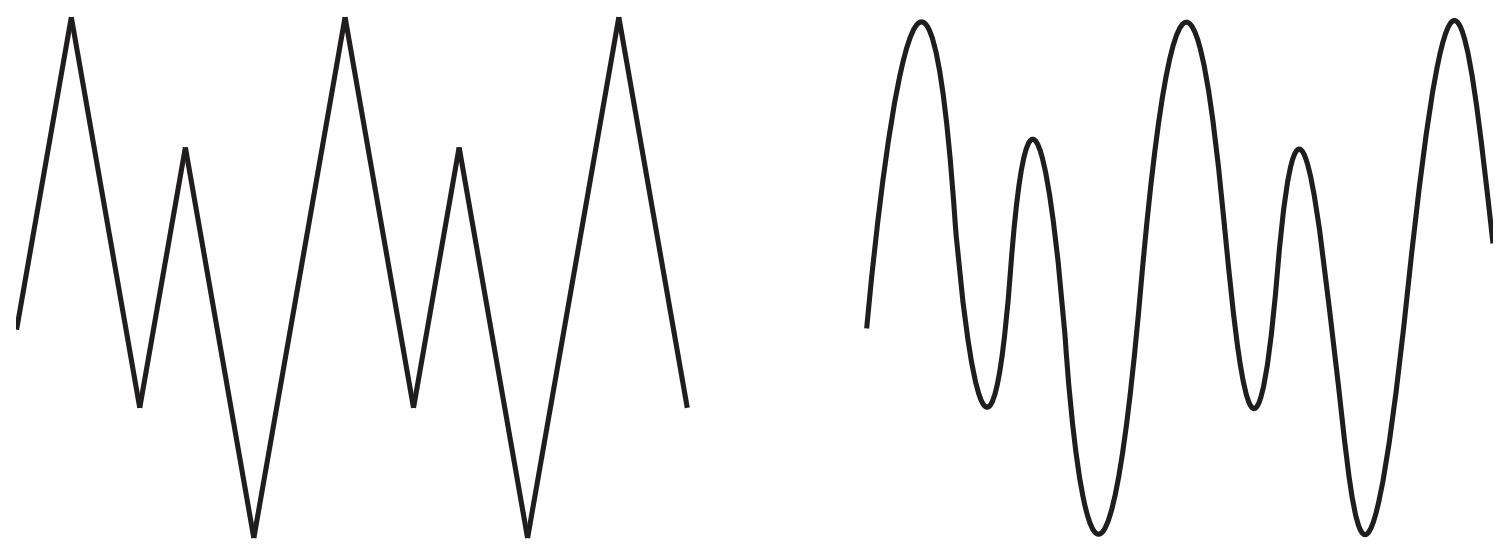

Figure 4.1. The zigzag (left) and wave (right) patterns used as stimuli during the recording session.

of the scale of the movement. After the initial practice trails, participants performed 3 blocks containing 5 repetitions of each pattern (in random order) with their eyes closed to limit visual experience.

\subsubsection{Motion capture}

All movements were recorded using an optical motion capture system (Vicon MX+ with 4 Vicon MX-F20 and 4 Vicon MX13+ cameras), at a sampling rate of $200 \mathrm{~Hz}$. Eleven $9 \mathrm{~mm}$ passive, reflective markers were placed on the participant's shoulders, right arm, right hand and waist (see Figure 4.2). To mark out the torso, two markers (RHSO and LSHO) were positioned on the top of the shoulders and two markers (RFWT and LFWT) were placed on the waist midway between the iliac crest and the rib cage. To track the movement of the right arm, an additional shoulder marker (RLSH) was placed on the lateral side of the right shoulder, with markers also positioned on the medial (RMEL) and lateral (RLEL) elbow and on the medial (RMWR) and lateral (RLWR) sides of the wrist. An additional marker was also placed on the lateral side of the upper arm (RUPA). Finally, to track the movement of the right hand, a marker was placed at the base of the middle finger (RFIN). To prevent participants moving their fingers independently from their hand, all participants performed the movement either holding a pen or with a clenched 


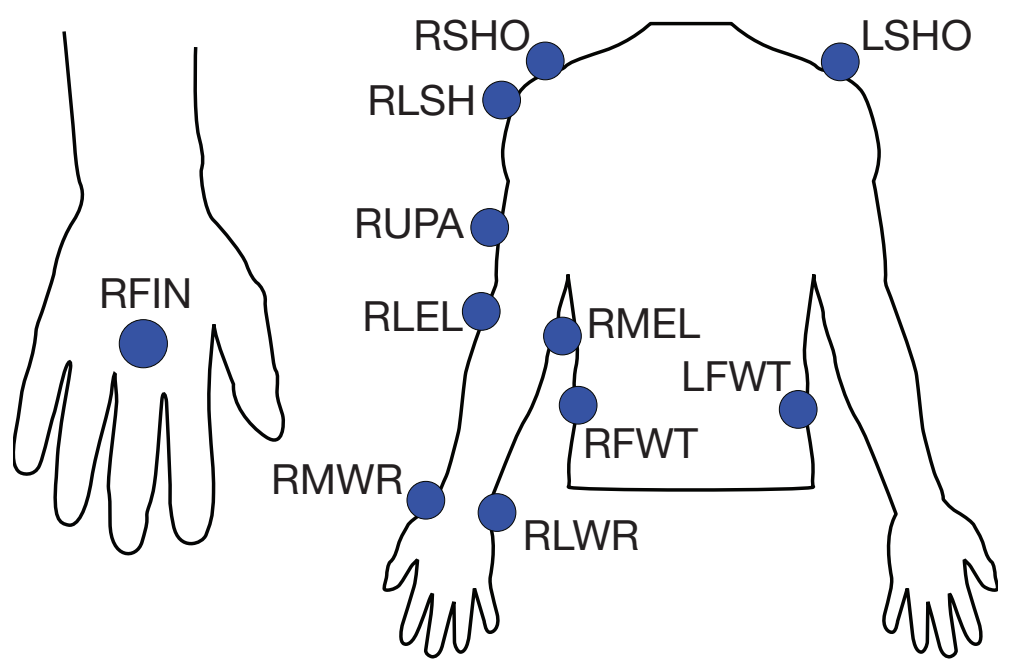

Figure 4.2. Marker positions for the 11 reflective markers used during the recording session.

fist, whichever was most comfortable.

\subsubsection{Movement description}

For the selected movements, the length of each movement (total distance travelled by the RFIN marker) was between $2.755 \mathrm{~m}$ and $6.513 \mathrm{~m}(M=4.090 \mathrm{~m}, S D=1.101)$. The average length of each long up-stroke was $0.431 \mathrm{~m}(S D=0.118$; range $=0.304$ to $0.700 \mathrm{~m})$, while the average length of each short up-stroke was $0.328 \mathrm{~m}(S D=0.096$; range $=0.196$ to $0.547 \mathrm{~m})$. The average length of the down-strokes was $0.421 \mathrm{~m}(S D=0.112$; range $=0.287$ to $0.664 \mathrm{~m})$. The duration of each movement was on average $7406.25 \mathrm{~ms}(S D=590.23$; range $=6450$ to $8775 \mathrm{~ms}$ ). The average duration of the upward movements was $721.67 \mathrm{~ms}$ $(S D=64.45 ;$ range $=622$ to 848$)$ for the long strokes and $649.50 \mathrm{~ms}(S D=69.25 ;$ range $=$ 573 to $855 \mathrm{~ms}$ ) for the short strokes. The average duration of the downward movements was $758.20 \mathrm{~ms}(S D=68.01 ;$ range $=640$ to $903 \mathrm{~ms})$.

\subsubsection{Test stimuli}

To create the stimuli, the motion capture data were first gap-filled using Vicon Nexus (Vicon Motion Systems, Oxford UK), and then resampled to $25 \mathrm{~Hz}$. The data were then ren- 

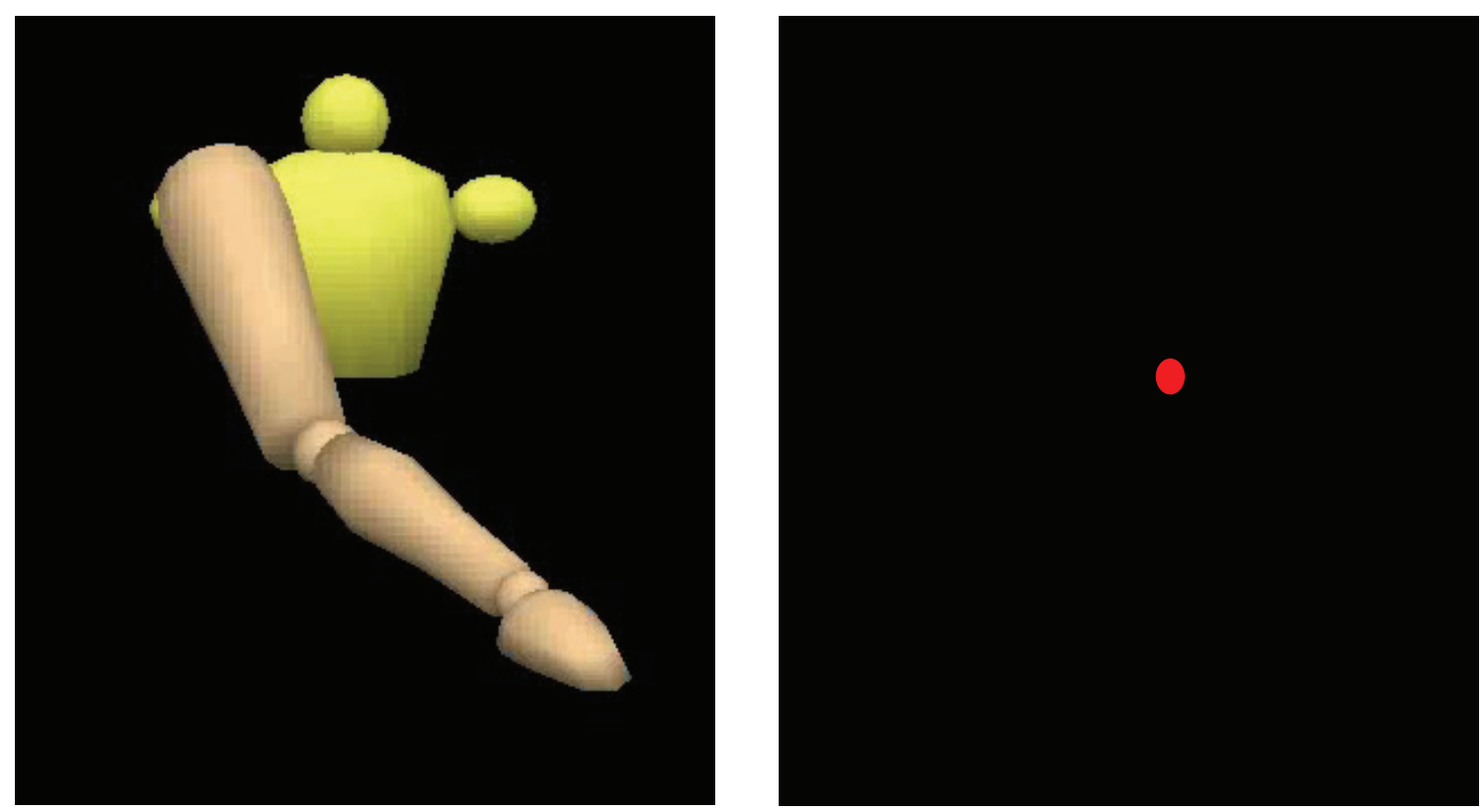

Figure 4.3. Example stimuli from Experiment 1 (left) and Experiment 2a and Experiment $2 b$ (right).

dered as a 3-D mannequin (see Figure 4.3 left), consisting of a torso, right arm and hand, using C-Motion Visual 3D (C-Motion INC., Rockville MD). The torso was defined using the shoulder (RSHO and LSHO) and waist (RFWT and LFWT) markers. The proximal and distal ends of the upper section of the right arm were defined using the right shoulder (RSHO) marker and the right elbow (RLEL and RMEL) markers. The forearm was defined using the right elbow and right wrist markers (RMWR and RLWR). The wrist markers and the right finger marker (RFIN) were used to define the proximal end and the midpoint of the hand, respectively.

To create the constant velocity stimuli, the raw trajectories were modified before the mannequins were created. The spatial and temporal positions for the local maxima and minima at the peaks and troughs of the movement trajectory were first determined, and new trajectories were then created using linear interpolation between these spatiotemporal points. This process creates straight trajectories that have a constant gradient and, therefore, movement along these trajectories is at a constant velocity. 


\subsubsection{Procedure}

The experiment was divided into two phases: a movement session and a test session. During the movement session, participants performed a movement task that was identical to the task employed during stimulus creation (see Section 4.2.1.2). The test session took place, on average, 7.30 days later (range $=7$ to 13 days). The task in the test session was to press the response button when the hand of the mannequin reached the apex of each upward movement. Participants were instructed to synchronise the button-press with the display as accurately as possible and were told that this may require them to anticipate the movement of the mannequin. Each participant performed 4 blocks containing 40 unique stimuli (composed of 20 trials for the constant velocity stimuli and 20 trials for the natural velocity stimuli, and equal numbers of wave and zigzag stimuli).

\subsubsection{Results}

The data were analysed by means of a $4 \times 2$ repeated measures ANOVA with the factors Block (1, 2, 3, and 4) and Velocity Profile (natural velocity, constant velocity). The Greenhouse-Geisser procedure was used to correct for violations of sphericity, where appropriate. The dependant variable of mean absolute timing error was used because it provides a good proxy for timing accuracy (Spray, 1986). Absolute timing error for a single response was calculated as the absolute time difference between the occurrence of the peak in the trajectory and the time at which the button was pressed. Button presses with an absolute timing error greater than $600 \mathrm{~ms}$ were regarded as too slow to reflect the process of interest and were, therefore, discarded from the analysis. This resulted in the exclusion of less than $1 \%$ of button-presses (including all button-presses did not change the pattern of results). The results showed a significant main effect of Block $\left(F_{3,51}=6.125, p=.009\right.$, $\epsilon=.549, \eta_{p}^{2}=.272$ ), suggesting that overall timing error decreased as the experiment progressed. In addition, there was a significant main effect of Velocity Profile $\left(F_{1,17}=107.850\right.$, $\left.p<.001, \eta_{p}^{2}=.978\right)$, suggesting that timing error for the constant velocity stimuli $(M=$ $169.81 \mathrm{~ms}, S D=47.76)$ was significantly higher than the timing error for the natural ve- 


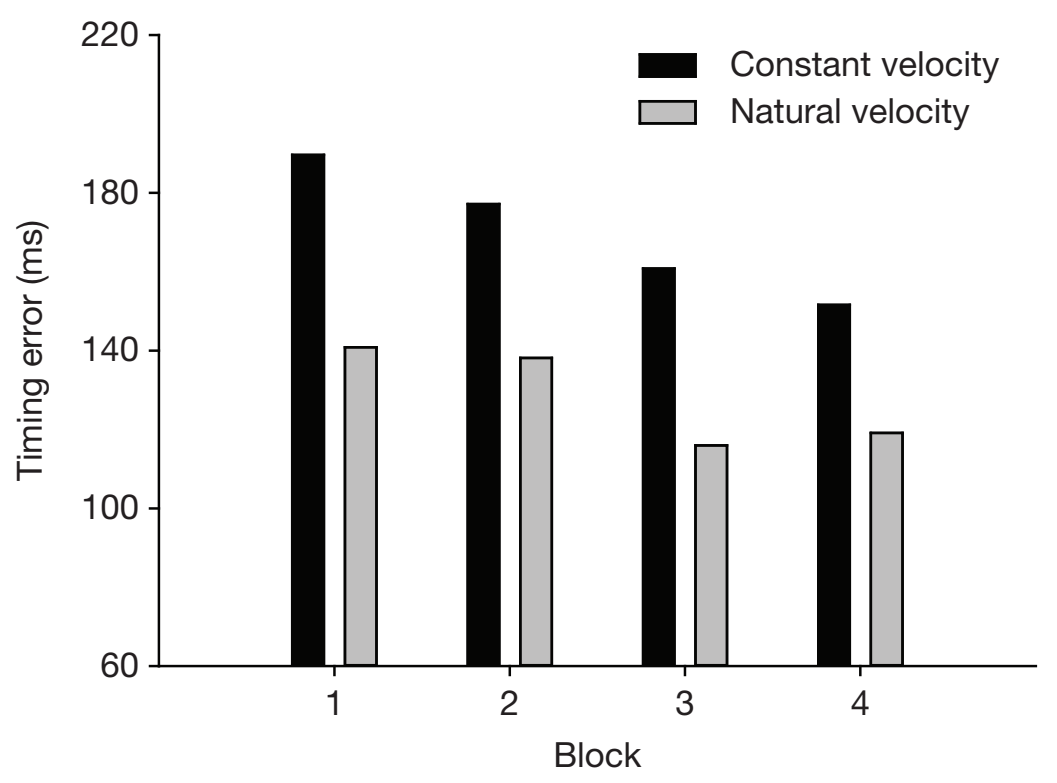

Figure 4.4. Absolute timing error for natural velocity stimuli and constant velocity stimuli for each of the four experimental blocks (Experiment 1).

locity stimuli $\left(M=128.52 \mathrm{~ms}, S D=40.51 ; M_{\text {diff }}=41.28,95 \% C \mathrm{C}=32.89\right.$ to 49.67$)$. Finally, the Velocity Profile $\times$ Block interaction was significant $\left(F_{3,51}=3.267, p=.029, \eta_{p}^{2}=.161\right)$ suggesting that the effect of Velocity Profile decreased as the experiment progressed (see Figure 4.4).

Taken together, these data demonstrate that synchronisation accuracy is not equal for stimuli with equal tempo. Rather, they suggest that the dynamics of the stimuli, or the velocity profile, is the primary determinant of synchronisation accuracy.

\subsubsection{Discussion}

The results of Experiment 1 show that synchronisation accuracy is not equal for stimuli that are identical in terms of the timing of the synchronisation points. Rather, participants show greater synchronisation accuracy for the natural velocity stimuli relative to the constant velocity stimuli. This suggests that, rather than the timing of the synchronisation points determining synchronisation accuracy, synchronisation accuracy is primarily determined by other aspects of the stimuli, such as the dynamics of the stimulus between the synchronisation points. This finding is consistent with the emulator hypothesis of 
action prediction because the natural velocity stimuli more closely match the observer's motor dynamics and the dynamics of the observer's forward model.

However, it is also the case that the data presented here can be explained without recourse to internal models. Rather than the dynamics of the stimulus being an important cue for predicting the timing of the synchronisation points, the mere presence of a deceleration phase before the occurrence of a synchronisation point, as is found in the natural velocity stimuli, might explain the enhanced synchronisation accuracy for the natural velocity stimuli. Experiment $2 \mathrm{a}$ and $2 \mathrm{~b}$ were designed to rule out this alternative explanation.

\subsection{Experiment $2 \mathrm{a}$ and $2 \mathrm{~b}$}

The aim of Experiment $2 \mathrm{a}$ and $2 \mathrm{~b}$ was to address the question of whether the mere presence of a deceleration phase can act as a reliable predictor for the timing of the synchronisation points. That is, Experiment $2 \mathrm{a}$ and $2 \mathrm{~b}$ were designed to test whether the presence of a deceleration phase is the primary cue that can account for the difference in synchronisation accuracy between the constant velocity stimuli and the natural velocity stimuli observed in Experiment 1.

According to the emulator hypothesis of action prediction, prediction of observed action relies on simulating the dynamics of the stimuli within the observer's motor system by using a forward model of the observer's action dynamics. However, before the forward model can be used to generate predictions about the observed action, the observer must map the observed action onto their own motor system in a manner that makes it possible to adequately replicate the dynamics of the stimulus. This mapping process would take time and, therefore, it may be possible to exploit this to test between the two alternative explanations for Experiment 1: the emulator account, which suggests that superior prediction accuracy for natural velocity stimuli is a result of the dynamics of the stimuli more closely resembling the dynamics of human action; and the alternative account, which suggests that the difference in prediction accuracy is due to the natural velocity 


\section{The effect of tempo and dynamic cues}

stimuli containing a deceleration phase prior to a synchronisation point.

One way to test between these accounts is to alter the amount of variability in the dynamics of the stimulus set. The two alternative explanations predict different patterns of results for this manipulation. The emulator account would predict that increasing stimulus variability would mean that more time is needed to learn to reconstruct the observed action, or to simulate the dynamics of the observed action, within the observer's motor system. This would reduce the effectiveness of the emulation process and this should disrupt the observer's ability to accurately predict the synchronisation points for the natural velocity stimuli. However, for the alternative account, the relevant cue that determines the difference in synchronisation accuracy between the natural and constant velocity stimuli is the presence of the deceleration phase before the synchronisation point, which is present in natural velocity stimuli. Thus, increasing the variability in the stimulus dynamics would not change the relevant cue; therefore, this alternative explanation predicts that this manipulation should not alter the difference in performance between natural and constant velocity stimuli.

Following this logic, we designed two further experiments where we varied the amount of variability in the stimulus set. Experiment 2a contained a low level of variability (the same amount as Experiment 1), while the variability was increased for Experiment 2b. Additionally, changes were made to the stimuli so that they were composed of only a single moving point. This was done to specifically isolate the effect of stimulus dynamics. In the case of full body stimuli, information such as arm extension or the level of flexion of the joints may act as additional cues to the timing of the synchronisation points. Similarly, motor experience might also add a further confound. ${ }^{1}$ Because of the changes made to the paradigm, Experiment 2a was used to determine whether the findings of Experiment 1 could be replicated with the modified paradigm.

To increase stimulus variability in Experiment $2 b$, new stimuli were used that, instead of being composed of the movements of 4 individuals (as in Experiment 1 and Experi-

\footnotetext{
${ }^{1}$ Subsequent testing revealed that the addition of a movement phase did not change the pattern of results; therefore, these data are not reported.
} 
ment 2a), were composed of the movements of 20 individuals (each of whom performed the movement task described in Experiment 1; see Section 4.2.1.2). Increasing the number of individuals used in stimuli creation increases the amount of variability in how the movements are performed. In both Experiment $2 \mathrm{a}$ and $2 \mathrm{~b}$, participants always viewed 40 unique movements per block (20 of each velocity profile); only the number of individuals used to create the 40 unique movements was changed. If action prediction relies on replicating the dynamics of the stimulus within the observer's motor system, then this manipulation should disrupt participants' ability to accurately predict the timing of the synchronisation points. However, this manipulation should have no effect on synchronisation accuracy if the relevant cue is the presence of a deceleration phase, as this has not been altered.

\subsubsection{Method}

\subsubsection{Participants}

For Experiment 2a, a total of 15 participants (12 females) volunteered to take part. All were right-handed ( $M=1.5$; range $=0.4$ to 2 ), as measured by the Edinburgh handedness inventory (Oldfield, 1971). The participants had an average age of 20.18 years (range $=19$ to 23 years). For experiment 2b, 23 participants (18 females) volunteered to take part. All were right-handed ( $M=1.7$; range $=0.6$ to 2$)$, and they had an average age of 21.49 years (range $=19$ to 28 years). All participants were free from movement difficulties, and had normal or corrected-to-normal vision.

\subsubsection{Stimuli}

The movements used to create the stimuli for Experiment 2a were identical to those used in Experiment 1. However, rather than using animated mannequins, the stimuli consisted of a single moving point-light (see Figure 4.3 right) that tracked the movement of the right hand marker (RFIN). For Experiment 2b, the same procedure was used to create the single moving point-light, but instead of using movements from 4 different individuals, we used 


\section{The effect of tempo and dynamic cues}

movements from 20 different individuals. As only one movement from each person was used, only exemplars of the wave movements were selected. Constant velocity stimuli were also created using the procedure described in Experiment 1 (see Section 4.2.2). The 20 individuals (15 female) used for stimulus creation had an average age of 25.35 years (range $=19$ to 50 years), and 19 were right handed $(M=1.2 ;$ range $=-1.2$ to 2$)$.

\subsubsection{Movement description}

The movements used for Experiment 2a were identical to those used in Experiment 1. For the movements used in Experiment 2b, the average length of the selected movements was $3.727 \mathrm{~m}(S D=0.932$; range $=2.293$ to $5.636 \mathrm{~m})$, with the large up-strokes measuring $0.405 \mathrm{~m}(S D=0.113$; range $=0.182$ to $0.611 \mathrm{~m})$ and the short up-strokes measuring $0.280 \mathrm{~m}(S D=0.094 ;$ range $=0.116$ to $0.474 \mathrm{~m})$. The average length of the down-strokes was $0.387 \mathrm{~m}(S D=0.096$; range $=0.228$ to $0.571 \mathrm{~m})$. The average duration of each movement was $6989.25 \mathrm{~ms}(S D=1337.71$; range $=4405$ to $8885 \mathrm{~ms})$. The average durations for the individual strokes were: $673.83 \mathrm{~ms}(S D=152.48$; range $=340$ to $902 \mathrm{~ms})$ for the long up-strokes; $580 \mathrm{~ms}(S D=130.06$; range = 278 to $808 \mathrm{~ms})$ for the short up-strokes; and $732.85 \mathrm{~ms}(S D=145.24$; range $=450$ to $972 \mathrm{~ms})$ for the down-strokes.

\subsubsection{Results}

\subsubsection{Experiment 2a}

Data analysis was performed on average absolute error by means of a $2 \times 4$ repeatedmeasures ANOVA with factors Velocity Profile (constant velocity, natural velocity) and Block (1, 2, 3, and 4). The Greenhouse-Geisser procedure was used to correct for violations of sphericity, where appropriate (we report uncorrected $d f$ s along with the $\epsilon$ value). The results showed a significant main effect for Velocity Profile $\left(F_{1,14}=57.934, p<.001\right.$, $\left.\eta_{p}^{2}=.805\right)$, suggesting that overall timing error for natural velocity stimuli $(M=171.83$ $\mathrm{ms}, S D=23.98$ ) was significantly higher than timing error for the constant velocity stimuli $\left(M=133.09 \mathrm{~ms}, S D=21.64 ; M_{\text {diff }}=38.74,95 \% C I=27.82\right.$ to 49.66$)$. Additionally, a 


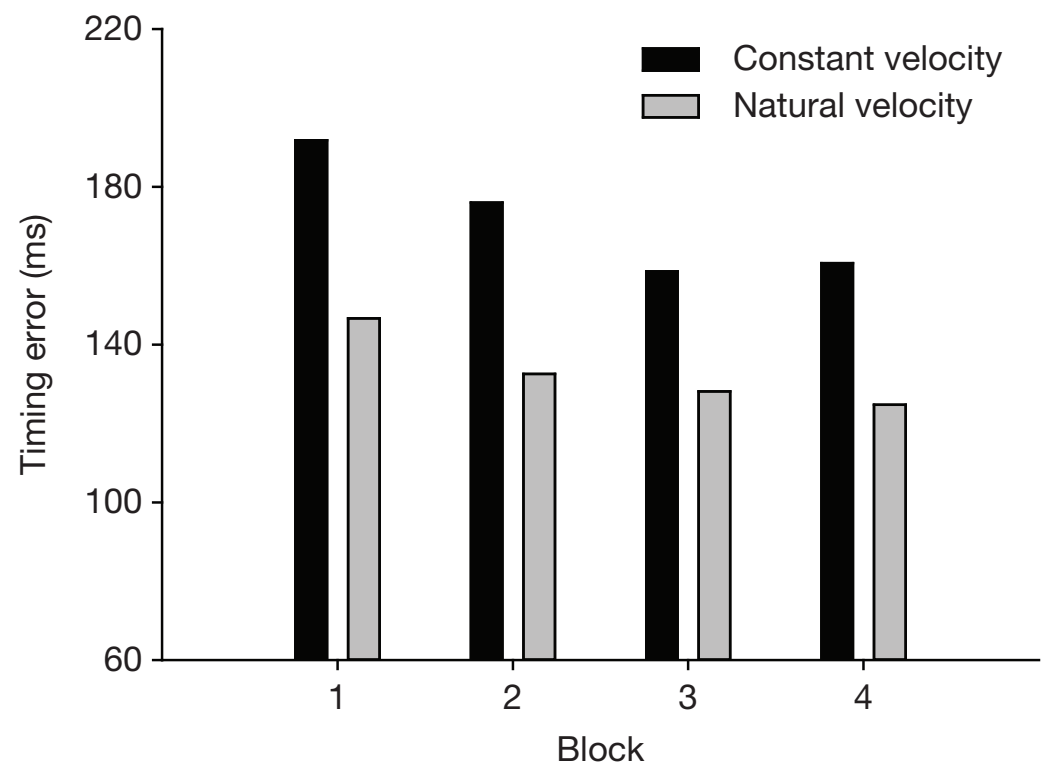

Figure 4.5. Absolute timing error for natural velocity stimuli and constant velocity stimuli for each of the four experimental blocks (Experiment 2a).

significant main effect of Block $\left(F_{3,42}=7.670, p=.007, \epsilon=.454, \eta_{p}^{2}=.354\right)$ was found, suggesting that overall timing error decreased over the course of the experiment. Finally, the Block $\times$ Velocity Profile interaction $\left(F_{3,42}=3.968, p=.014, \eta_{p}^{2}=.221\right)$ was also significant, suggesting that the effect of Velocity Profile was not consistent throughout the experiment, and instead decreased as the experiment progressed (see Figure 4.5). As Experiment $2 \mathrm{~b}$ only used wave stimuli, we repeated the analysis selecting only exemplars of the wave pattern. This did not change the pattern of results.

\subsubsection{Experiment $2 b$}

The analysis revealed a significant main effect of Block, suggesting that overall timing error decreased as the experiment progressed $\left(F_{3,66}=5.536, p=.010, \epsilon=.580, \eta_{p}^{2}=.201\right)$. Neither the main effect for Velocity Profile $\left(F_{1,22}=1.296, p=.267, \eta_{p}^{2}=.056\right)$ nor the Block $\times$ Velocity Profile interaction $\left(F_{3,66}=2.242, p=.092, \eta_{p}^{2}=.092\right)$ were significant. This suggests that there was no difference in overall timing error between the constant velocity stimuli $(M=157.42 \mathrm{~ms}, S D=32.41)$ and the natural velocity stimuli $(M=152.39 \mathrm{~ms}$, $S D=41.16 ; M_{\text {diff }}=5.03,95 \% C I=-4.13$ to 14.19$)$. These data are displayed in Figure 4.6. 


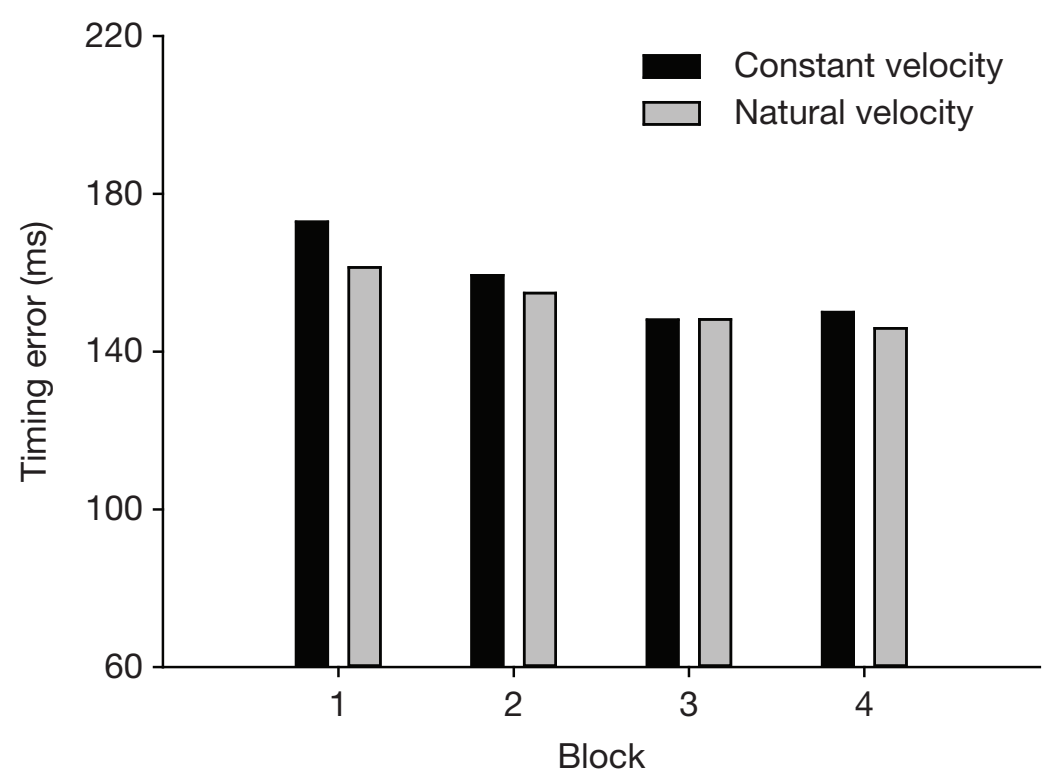

Figure 4.6. Absolute timing error for natural velocity stimuli and constant velocity stimuli for each of the four experimental blocks (Experiment $2 b$ ).

Furthermore, a direct comparison of the size of the synchronisation advantage (i.e., the mean timing error difference between natural and constant velocity stimuli collapsed over block) showed that the synchronisation advantage was significantly greater in Experiment 2a $(M=38.739 \mathrm{~ms}, S D=19.712)$ compared with Experiment $2 \mathrm{~b}(M=5.028 \mathrm{~ms}, S D=$ $21.179 ; M_{\text {diff }}=33.71,95 \% C I=19.83$ to $\left.47.59 ; t_{36}=4.926, p<.001\right)$.

\subsubsection{Discussion}

The primary aim of Experiment $2 \mathrm{a}$ and $2 \mathrm{~b}$ was to determine whether deceleration could act as a robust predictor for the timing of the synchronisation points. The results of Experiment 1 suggest that tempo alone does not determine synchronisation accuracy. Rather, synchronisation accuracy is determined, in part, by the dynamic properties of the stimulus between the synchronisation points. While this finding is consistent with the emulator hypothesis, the results are also consistent with participants merely responding to the deceleration that occurs before the synchronisation points. Experiment $2 \mathrm{a}$ and $2 \mathrm{~b}$ were designed to test the plausibility of this alternative explanation.

The results of Experiment $2 \mathrm{~b}$ show that deceleration is not an unambiguous cue. While 
participants were still able to accurately predict the timing of the synchronisation points when there was little variability in the stimuli (Experiment 2a), increasing the variability (Experiment $2 \mathrm{~b}$ ) resulted in the human velocity profile stimuli being no more predictable than the constant velocity profile stimuli. This suggests that the results of Experiment 1 cannot be explained by participants merely responding to the presence of deceleration in the stimuli. Rather, deceleration in the stimulus is only an effective cue when participants are given the opportunity to learn the dynamics of the stimuli.

\subsection{General discussion}

The aim of this study was to examine whether tempo was a primary determinant of synchronisation accuracy. Previous studies examining action prediction have employed rhythmic stimuli without controlling for production tempo (Flach et al., 2003; Chapter 2). Therefore, it is possible that the observed differences in synchronisation accuracy, including the authorship effect, might be explained by differences in the rhythmic properties of the stimuli and differences in how readily participants can synchronise with a particular tempo.

In Experiment 1, we compared synchronisation accuracy for two sets of stimuli that were matched for tempo and that differed only in terms of the dynamic properties of the stimuli between the synchronisation points. If tempo is a primary determinant of synchronisation accuracy, then we should expect to find no difference in synchronisation accuracy for tempo-matched stimuli. The results show that this is not the case. Instead, the primary determinant of synchronisation accuracy was the velocity profile of the stimuli, with significantly better synchronisation accuracy for natural velocity stimuli relative to constant velocity stimuli.

While the results of Experiment 1 are consistent with the emulator hypothesis of action prediction, it is also possible that participants merely started the process of response execution when they observed the stimulus begin to slow before the synchronisation points occurred. To rule out this explanation, Experiment $2 \mathrm{a}$ and $2 \mathrm{~b}$ examined whether the mere 


\section{The effect of tempo and dynamic cues}

presence of deceleration before the synchronisation points was sufficient for enhanced synchronisation accuracy.

The results of Experiment $2 \mathrm{a}$ and $2 \mathrm{~b}$ suggest that synchronisation accuracy is only better for natural velocity stimuli when stimulus variability is low (Experiment 2a). When stimulus variability is increased (Experiment $2 b$ ), synchronisation with natural velocity stimuli is no more accurate than synchronisation with constant velocity stimuli. This suggests that the mere presence of a deceleration phase in the stimuli used in Experiment 1 is not sufficient to explain the results.

These results are consistent with the hypothesis that, during action prediction, observers recreate the dynamics of the stimulus using an internal motor representation, which can then be used to generate predictions about its unfolding dynamics. When stimulus variability is high, the process of internally replicating the dynamics of the stimulus is harder because there is less opportunity for learning; when stimulus variability is low, it is easier for the observer to internally model the dynamics of the stimulus. Once the dynamics of the stimulus have been replicated in the forward model, the observer is able to use this forward model to generate predictions about the timing of the synchronisation points, which results in superior synchronisation accuracy for natural velocity stimuli over stimuli that do not exhibit human movement characteristics.

However, it should be noted that our results do not rule out the importance of the deceleration cue as a primary driver of the authorship effect reported by Flach et al. (2003) and in Chapter 2. Rather, our results suggest that before deceleration can be used as a cue, participants need an opportunity to learn to replicate the dynamics of the stimulus. In the present study, all participants viewed actions performed by other people and, therefore, they could only gain the required knowledge through repeated visual exposure to the stimulus over the course of the experiment. When examining the self/other synchronisation difference (e.g., Flach et al., 2003; Chapter 2) there is an additional source of information that participants have access to-they have internal access to their own action dynamics. Therefore, it may be this difference in access to internal information 
that drives the authorship effect.

\subsection{Conclusions}

The results of this study demonstrate that tempo is not a primary determining factor for synchronisation accuracy. Synchronisation accuracy was not equal for tempo-matched stimuli. Rather, synchronisation was more accurate for stimuli that contained dynamic cues between synchronisation points. However, these dynamic cues are not unambiguous, and participants need to learn the regularities of the stimulus before they can exploit these cues for the purposes of action synchronisation. Taken together, these results are consistent with the hypothesis that observers predict actions by simulating the dynamics of the stimulus within their own motor system.

\subsection{References}

Csibra, G. (2008). Action mirroring and action understanding: an alternative account. In P. Haggard, Y. Rossetti \& M. Kawato (Eds.), Sensorimotor foundations of higher cognition (pp. 435-459). New York: Oxford University Press.

Flach, R., Knoblich, G., \& Prinz, W. (2003). Off-line authorship effects in action perception. Brain and Cognition, 53(3), 503-513.

Flash, T., \& Hogan, N. (1985). The coordination of arm movements: an experimentally confirmed mathematical model. The Journal of Neuroscience, 5, 1688-1703.

Fraisse, P. (1982). Rhythm and tempo. In D. Deutsch (Ed.), The psychology of music (pp. 149-180). New York: Academic Press.

Golnaraghi, M., \& Kuo, B. C. (2010). Automatic control systems (9th ed.). Hoboken NJ: Wiley.

Kawato, M. (1999). Internal models for motor control and trajectory planning. Current Opinion in Neurobiology, 9, 718-727. 
Keller, P. E., Knoblich, G., \& Repp, B. H. (2007). Pianists duet better when they play with themselves: on the possible role of action simulation in synchronization. Consciousness and Cognition, 16(1), 102-111.

Oldfield, R. C. (1971). The assessment and analysis of handedness: the edinburgh inventory. Neuropsychologia, 9(1), 97-113.

Rizzolatti, G., \& Craighero, L. (2004). The mirror-neuron system. Annual Review of Neuroscience, 27(1), 169-192.

Spray, J. A. (1986). Absolute error revisited: an accuracy indicator in disguise. Journal of Motor Behavior, 18(2), 225-238.

Viviani, P., \& Stucchi, N. (1992). Biological movements look uniform: evidence of motorperceptual interactions. Journal of Experimental Psychology: Human Perception and Performance, 18(3), 603-623.

Wilson, M., \& Knoblich, G. (2005). The case for motor involvement in perceiving conspecifics. Psychological Bulletin, 131(3), 460-473.

Wolpert, D. M. (1997). Computational approaches to motor control. Trends in Cognitive Sciences, 1(6), 209-216. 


\section{Part III.}

A theoretical framework for joint action 



\section{CHAPTER 5}

\section{From action control to joint action}

\subsection{Introduction}

Traditional philosophical analyses of joint action have focused on such problems as differentiating truly joint actions from seemingly similar actions that are not joint actions. Consider the example, from Searle (1990), of a group of people converging on a common point in a park. This behaviour could either be a collective behaviour or it could be a collection of individual behaviours. What differentiates the two are the intentions that cause the behaviour. If the people each have the intention "it is raining and I am running to shelter", then it is merely a collection of individual behaviours. For it to be a collective behaviour, or joint action, a different sort of intention is required. This might take the form of a collective intention such as "we intend to get ready to perform a ballet by means of me running to the gazebo" (Searle, 1990), or the form of a shared intention consisting of individual intentions to perform together and appropriate interrelations between the individual intentions (Bratman, 1993).

The purpose of these shared or collective intentions is to allow the individual agents to 


\section{From action control to joint action}

coordinate their actions in such a way that they successfully carry out the joint action. To use the paradigm case of joint action put forward by Bratman (1993): When deciding to paint a house together, our shared intention to paint the house together will result in behaviour that allows each of us to negotiate our part in the joint action. I can plan to mix the paint while I can tell you to get the paint brushes. Here, language can also play a role in coordinating our actions so that we can realise our joint goal. But while this type of account may be well suited to these types of joint actions, it may not work equally well for all instances of joint action.

The insufficiency of an account based on meshing of sub-plans (see Bratman, 1993) can be illustrated by taking as the paradigm case a different sort of joint action. The joint actions considered in this paper are actions such as playing music together, dancing together, or coordinating actions on a sports field. By examining such examples, we can draw out important features of joint action that have been overlooked (or at least deemphasised) in earlier accounts. These types of joint actions differ from those examined by traditional analyses in at least three ways: they face heavy time constraints, actions need to be coordinated without the use of language, and the interactions are transparent. By considering each of these dimensions we are able to distinguish what we might call sensory-motor coordinated joint action ${ }^{1}$ from interactions where co-actors can share intentions, negotiate roles, and explicitly plan a strategy to carry out the joint action. Our framework does not aim to provide an account of these three dimensions; rather, these dimensions are used to define our explanatory target (i.e., sensory-motor coordinated joint action).

First, the joint actions that our framework aims to explain may face heavy time constraints. Time constraints mean that action must be coordinated rapidly. Rather than responding to the actions of their co-actors, actors must anticipate and, hence, predict the

\footnotetext{
${ }^{1}$ By labelling our target phenomenon sensory-motor coordinated joint action we do not mean to say that only joint actions such as ensemble music and dance performance are coordinated by means of sensory-motor mechanisms. However, these types of interactions provide the clearest example of joint actions coordinated by means of sensorymotor mechanisms and, therefore, this is where we target our account.
} 
actions of their co-actors so that they can plan and execute their actions in time. Thus, our framework offers an account of how it is possible for actors to predict the actions of their co-actors.

Second, time constraints may make it impossible (or at least difficult) to use linguistic communication to update sub-plans in a manner possible in more deliberative, less timeconstrained, joint actions such as Bratman's (1993) example of painting a house. For example, language can be used off-line to decide on the time and place at which we will play a piano duet and to negotiate who will play the primo and who will play the secondo; however, for the performance to be successful, what is needed is a means of rapid, online coordination of action that does not and, because of time pressures, cannot depend on language. As with the challenge posed by time pressures, the inability to make use of linguistic coordination can be solved by the ability of actors to predict or anticipate the actions of their co-actors, and the ability to coordinate their action execution on the basis of these predictions. Therefore, rather than focusing on collective intentions or the nature of the set of interrelated sub-plans required to support joint action, the present paper attempts to provide an account of the prediction and planning mechanisms that make this ongoing coordination possible. The distinction between those types of joint actions that make use of language for coordination and those that do not is not categorical. Rather, they represent either end of a continuum with most instances of joint action making use of a mixture of linguistic and non-linguistic coordination. Indeed the nature of this mixture may be different for different instances of the same activity. Furthermore, while the requirements for a rapid and non-linguistic means for coordination both arise from the time pressures inherent in the examples of sensory-motor coordinated joint action we have chosen (i.e., music and dance performance), this need not be the case. Certain interactions may face time pressures but may also make extensive use of linguistic means of coordination-for example, calling plays on the sports field-while other interactions may make use of non-linguistic means of coordination when not facing time pressures. However, because sensory-motor coordinated joint actions can be coordinated without 


\section{From action control to joint action}

language, our framework must make do without an account of how language can be used to coordinate joint actions.

The third dimension on which to characterise the interactions we aim to explain-sensory-motor coordinated joint actions-is transparency. Our account is aimed at transparent interactions where co-actors are free to focus on the product of the interaction (e.g., the piece of music that is produced) rather than the process of interaction (e.g., the initiation and exchange of sub-plans through linguistic communication). To clarify what is meant by transparent, it is useful to consider the example used by Heidegger (and picked up by Dreyfus, 1991, and Clark, 2003), of a carpenter's hammer. When the carpenter uses the hammer, she does not encounter the hammer as an object in and of itself, but instead engages with the tool transparently, that is, in such a way so that she "sees through" the hammer to the task or goal of nailing a picture to the wall. A novice might instead encounter the hammer as opaque where the hammer is not invisible but rather remains the focus of attention. In the same sense, the interactions we aim to describe are those where actors coordinate their actions transparently. The coordination process is invisible, and only the ultimate task or goal enters awareness. Of course, transparent and opaque interactions represent two ends of a continuum with many interactions being at times transparent and at times opaque. Moreover, interactions may also change from opaque to transparent as expertise is gained; for example, as the novice pianist becomes an expert. While not all sensory-motor coordinated joint actions occur transparently, transparency is a feature of the skillful joint coping found in, for example, expert musicians, and thus transparency might be an important part of the phenomenology of joint action.

We aim to employ our account of joint action to provide an initial sketch of the mechanisms that might underlie the peculiar phenomenology associated with sensory-motor coordinated joint action. Actors engaged in these interactions report a variety of experiences ranging from feelings that arise from skillful joint coping, which Sawyer (2006) has labelled "group flow", to more extreme cases where actors report a blurring of the 
self-other distinction, described variously as a "blurring of self-awareness" (McNeill, 1995) and "boundary loss" (Hanna, 1977, p. 199). Feelings of group flow may be more common for skilled musicians who describe these experiences as "striking a groove" (Berliner, 1994) or simply "groove" (Monson, 1996), as they more often engage in transparent joint action.

Although the phenomenology of action and agency has recently received more attention within the empirical and theoretical literature (e.g., Carruthers, 2010; Frith, Blakemore, \& Wolpert, 2000; Pacherie, 2008; Wegner, Sparrow, \& Winerman, 2004), the phenomenology of joint action has been relatively unexplored. Recent accounts of individual agency and the phenomenology of action have been based on predictive mechanisms, such as those employed in action control (e.g., Frith et al., 2000; Pacherie, 2008). By basing our framework of joint action on a model of the predictive mechanisms in action control, we aim to also extend it to the phenomenology of joint action. While a detailed account of the mechanisms that underlie experiences such as group flow is not yet possible, they may be mediated by the predictive mechanisms that underlie joint action coordination and, therefore, the framework presented here might provide a starting point for such an account.

The approach to joint action coordination that we present here draws on two recent accounts. First, Csibra (2008), in developing a critique of mirror neuron-based approaches to action understanding (Rizzolatti, Fogassi, \& Gallese, 2001; see also Gallese \& Goldman, 1998), has suggested that mirror neurons form part of a system for predicting the actions of others on the basis of already understood goals. He proposes that these predictions may play an important role in joint action. Currently, however, mirror neuronbased approaches rely on empirical data and interpretations that remain controversial. Furthermore, Csibra's (2008) account has not as yet been developed into a full account of joint action coordination. The framework we present is, however, not based on the mirror-neuron system. Rather, our framework is based on behavioural evidence. Similarly, our account suggests that the goals (or goal conjectures) we assign to others plays 


\section{From action control to joint action}

an important part in predicting their action. However, we extend Csibra's (2008) account to show that the goals we assign to others has a direct influence on action planning and execution. Moreover, we integrate this notion into a larger framework that explains how sensory-motor coordinated joint action is possible.

Second, influential work by Sebanz, Bekkering, and Knoblich (2006) has begun to lay the groundwork for understanding the mechanisms that underlie joint action. They highlight the importance of being able to share representations, predict actions, and integrate the predicted effects of other's actions with one's own action planning. In particular, Sebanz et al. (2006) highlight the importance of joint attention for creating a "perceptual common ground" that is important for not only initiating a joint action but also for coordinating actions once joint action has begun. Drawing from studies that show that actions are represented in terms of their goals, Sebanz et al. (2006) suggest that action observation during joint action can be used to infer the goals of co-actors. Action observation can also serve a secondary purpose by supporting the prediction of action or the prediction of action outcomes. Furthermore, Sebanz et al. (2006) suggest that co-representation of a co-actor's task also assists in action prediction, and that joint action coordination relies on the ability to integrate these predictions with one's own action execution.

While our account also highlights the importance of prediction, the framework we present and the framework of Sebanz et al. (2006) are built on different foundations. Our approach relies on extending well understood models of action control into the social domain. We provide a detailed account of how aspects of the action control system (including inverse and forward models) may be extended to the case of joint action coordination. Predictive models lie at the core of recent theories that have been used to explain how individuals plan and execute their actions. For instance, predictive models play an important role in understanding how people coordinate actions between multiple effectors-for example, manipulating an object with two hands (e.g., Witney, 2004) or coordinating movement between the hand and the eyes (e.g., Miall \& Reckess, 2002). The framework outlined here extends these ideas from the case of individual action control to 
the case of joint action control by suggesting that similar predictive mechanisms underlie both action control and coordination within individuals and action coordination between individuals. Thus, our approach allows us to reconstruct and extend on earlier work in the field.

The outline of the paper is as follows. First, we provide a basic introduction to control theory and explain how goal-directed behaviour is implemented in engineered systems, and we provide an introduction to Grush's (1997) emulator theory of representation that our account draws on (Section 5.2). Next, we outline how notions from control theoryin particular, predictive models and emulators-have been employed in theories of how humans plan, execute, and control motor actions (Section 5.3). Following this, we introduce our framework for joint action coordination (Section 5.4). We suggest that the action control system is also used off-line during joint action thereby allowing individuals to emulate and predict the actions of their co-actors. We present evidence that, during joint action, individuals represent the goals of their co-actors in a manner that is functionally equivalent to the way they represent their own goals (Section 5.4.1)—we refer to this as task co-representation. The interpretation we support for some of the data discussed in this section is controversial; therefore, we will also outline two alternative explanations: the actor co-representation account, which holds that the specifics of a co-actor's goals are not represented, only that the co-actor is responsible for the other half of the task, and the spatial coding account, which claims that the phenomena reported are not social in nature. However, these alternatives need not be mutually exclusive, and we argue that on balance there is evidence to support the task co-representation account, at least in some situations, but this does not discount the fact that the alternatives might be better at explaining other findings. We argue that goals can then be fed into the action control system, taken off-line, in order to, first, generate the control commands that would be issued in order to realise that goal and, second, to generate predictions about how the action will unfold and the sensory consequences the action will produce. We also present evidence from action synchronisation studies, including our own work, that suggests how 


\section{From action control to joint action}

it is possible to reconstruct these control commands (Section 5.4.2). Finally, we show that through co-representation of co-actors' goals, and by emulating their actions to predict how these goals will be realised, individuals are able to form joint action models that are functionally equivalent to individual action models. This allows for the coordination of joint action in a manner that is functionally equivalent to individual action coordination (Section 5.4.3). If joint action coordination and individual action control are functionally equivalent, then it might be possible that functionally equivalent mechanisms underlie both the phenomenology of individual action and the phenomenology of joint action.

When skilfully executed, sensory-motor coordinated joint actions-rapid, transparent interactions that do not rely on language-have a distinctive feel that is often not present in the case of more deliberative coordination. Our framework is based on models of individual action control, and these models have also been successfully extended to explain a range of empirical findings within the phenomenology of individual action. Thus, in Section 5.5 we attempt to extend our account to explain the phenomenology of joint action and experiences such as group flow, which are often reported by, for example, professional musicians and sports-people. We provide an initial sketch of what such an extension might look like.

\subsection{Predictive models in control theory}

Recent models of action control have relied heavily on notions borrowed from control theory. Our account, similarly, relies on these notions as well as the emulator theory of representation put forward by Grush (1997). Thus, before introducing action control, we first provide an introduction to the concepts and terminology of control theory and emulators. Goal-directed behaviour can be implemented in engineered systems by means of closed-loop control. A simple example is a room heater consisting of a heating element, a temperature control, and a temperature sensor (thermostat). Once a temperature has been selected, the system controller transforms the target room temperature, or goal state, into commands that are sent to the target system (the heating element). These commands 
consist of, for example, specifications for the amount of current to be passed through the heating element. The controller is said to perform an inverse mapping from the goal state (target room temperature) to control commands. The control commands produce the behaviour required to bring about the desired goal state in the target system. The target system performs a forward mapping from control commands into the goal state. In closed-loop, or feedback control, the temperature sensor can be used to measure the current state of the system (room temperature) to compare it to the goal state. Any discrepancy between the current system state and the goal state can be fed back to the system controller so adjustments can be made to the control signals-more current can be passed to the heating element if the room is too cold or less current if it is too hot. The advantage of feedback control is that it allows the system to adjust its behaviour to changes in the environment or to compensate for inaccuracies in the initial control commands that would otherwise need to be precisely defined at the onset.

A major obstacle in implementing feedback control is that the measurements received from the sensors can be inaccurate because of temporal delays in receiving feedback or due to inherent sensor inaccuracies. One can compensate for these inaccuracies by using a model, or emulator, of the target system's behaviour. Grush (1997) has illustrated the usefulness of emulators by using the example of a heater on a space station located a significant distance away from the controller placed on Earth. Because there is a limit on how quickly radio signals can travel between the controller and the space station, feedback received from sensors will actually provide a measure of the temperature on the space station as it was at some time in the past. Any commands sent on the basis of these measurements may be inappropriate for the system's current state. For instance, by the time the controller issues a command to turn off the heaters the temperature on the space station will be well in excess of the goal temperature. The temperature will then begin to fall until it falls well below the goal temperature and it will continue to fall until the controller eventually sends a signal to restart the heaters. Rather than maintaining the goal temperature, feedback delays will result in the temperature continually oscillating 


\section{From action control to joint action}

around the goal.

To overcome this problem, Grush (1997) suggests that an emulator, or forward model, can be trained to exactly mimic the forward mapping behaviour of the space station — that is, it maintains the relationship between particular control signals and particular changes in temperature. When the controller issues control signals, one copy of the signal is sent to the distant space station while another copy is sent to the emulator. The emulator responds to the control signals just as the real space station would, and the feedback from the space station can then be substituted with predicted feedback from the emulator. As the emulator is also located on Earth the feedback will not be delayed.

A second problem that can arise during feedback control is that the sensors can be inaccurate. For example, temperature sensors in the room may contain random noise so that they never truly reflect the actual temperature of the room. If these sensor readings are used for feedback control, then the behaviour of the system would also be susceptible to sensor noise. This problem can also be overcome by means of an emulator. However, unlike the space station example, the actual sensor readings are not completely substituted by the predicted temperature readings from the emulator. Rather, the predicted and actual sensor readings can be combined in order to produce a more accurate estimate of the real room temperature. The contribution of each value (predicted and actual) to the final feedback value can also be varied according to the expected accuracy of each measurement (Golnaraghi \& Kuo, 2010).

The power of emulators is two-fold. First, they provide a means of controlling a system in real time under conditions of delayed feedback and imperfect knowledge of the system's state. In the next section, we show how these functions may be important for action control. Second, the whole system can be taken off-line and the emulator can be used as a model of the target system's behaviour without the target system actually producing any output. This can be done by only sending control commands to the emulator and not to the target system. We will argue that this occurs during joint action where an individual's action control system can be taken off-line and used as an emulator of another 
agent's action control system. This allows individuals engaged in joint tasks to predict and anticipate the actions of their co-actors.

\subsection{Prediction and action control}

Emulators such as those described in the preceding section have been implicated in many different areas of cognition (see Grush, 2004); however, the strongest evidence for the explanatory utility of emulators comes from action control. The model of action control developed by Wolpert (1997) relies heavily on notions borrowed from control theory, including emulators. As with the heater example, an inverse model is used for computing what motor commands are needed in order to bring about a particular goal state given such factors as the current state of the limb (its position, joint angles, and so forth). The motor command is then sent to the limbs in order to produce the actual movements. In addition, a copy of the motor command is sent to an emulator. The emulator can be divided into two parts: the forward output model, used to predict the sensory consequences that will result from performing the action, and the forward dynamic model, used to predict the change in limb position.

The predictions generated by these forward models can be used for several purposes. For instance, forward modelling can be used to anticipate and compensate for the sensory effects created by movements of, for example, the eye. This can be used to selectively filter the sensory consequences of self-movement thereby making it possible to distinguish between the sensory effects caused by self-movement from those caused by movement in the environment. Forward models also allow the action control system to bypass feedback delays, which allows for fine-tuning of movements without having to wait for actual sensory feedback from the periphery. Furthermore, because sensory signals might not always provide accurate information about the current state of the system, sensory signals can be combined with forward models in order to derive better state estimates (Miall \& Wolpert, 1996; Wolpert, 1997).

The ability to predict the sensory consequences of an action may also play a role in ac- 


\section{From action control to joint action}

tion planning. For instance, ideomotor theories of action planning suggest that actions are planned on the basis of predicted effects. The link between action planning and prediction has its origins in the development of action control. Hommel (2003; see also Elsner \& Hommel, 2001) has suggested a two-stage model to explain the development of voluntary action control. In the first stage, actions are generated in a random fashion. These actions lead to perceivable changes in the environment and the body. These action effects, or perceptions, then become associated with the action used to produce them. This process is analogous to the training of the inverse and forward models that, for example, Wolpert (1997; see also Wolpert \& Kawato, 1998) has suggested underlie action control. Once action and action-effects have become strongly associated, particular actions can be selected by activating the perceptual codes that correspond to the desired goal. Due to the strong association between perceptual codes and action codes, activation of a perceptual code partially activates its associated motor code. This, in effect, allows action selection on the basis of predicted perceptual effects. Furthermore, this suggests that action both creates perception and is the consequence of perception, and that perception is both the stimulus for action and the result of action. This implies that perception and action must share a common representational code (Hommel, 2009; Hommel, Müsseler, Aschersleben, \& Prinz, 2001, Prinz, 1997). Common coding of perception and action also has important implications for joint action. With no clear division between perception and action codes, this suggests that the same representations are involved in both producing actions and perceiving actions (e.g., see Knoblich \& Sebanz, 2008). This framework has also been used to explain a number of perception-action interactions including, for example, the Simon task, which we describe in Section 5.4.1.

\subsection{Common mechanisms for individual and joint action}

In the section that follows (Section 5.4.1) we will outline evidence that suggests that observers represent the subgoals of their co-actors within their own motor systems by performing a transformation analogous to an inverse model by transforming the goals of 
others into action representations. We refer to this process as task co-representation. We will also outline two alternative explanations for some of the data-from studies on the joint Simon effect-used to support the notion of task co-representation, namely actor co-representation and spatial coding. However, we will argue that these accounts are not mutually exclusive and that, on balance, the evidence suggests that task co-representation occurs in at least some situations. Following this (Section 5.4.2), we will argue that the observation of actions can assist in the reconstruction of the motor codes performed by the inverse model. Once reconstructed, these motor codes can be combined with forward models to generate predictions about the unfolding motor acts produced by observed agents or to predict the sensory consequences of actions performed by observed agents. Finally, we argue that the ability to predict the actions of others allows co-actors to integrate actions across individuals in a manner similar to how actions are integrated within individuals (Section 5.4.3).

\subsubsection{Co-representation of subgoals in joint tasks}

In order to engage in joint action, it is useful to know what actions one's co-actor will select to realise a particular goal. To do this, it is necessary to represent their goal in some manner. By definition, our own action goals affect our action control system; however, recent evidence suggests that the goals of other agents might be represented within our own motor systems in such a way that they might, similarly, also affect action control.

Evidence from monkey electrophysiology and human neuroimaging has shown that the motor system is engaged not only during action production, but also during the passive observation of action. That is, when observing actions performed by others, the motor system of the observer is activated in a manner similar to the way it would be activated if they were performing the action themselves (Rizzolatti \& Craighero, 2004). This finding has ordinarily been used to support the notion that observed actions are coded in terms of the motor commands used to produce those actions. However, what is most interesting about this neural system, known as the mirror-neuron system, is that it is sensitive to 


\section{From action control to joint action}

the goals of the observed agent. For example, Umiltà et al. (2001) found a population of mirror neurons that exhibit selective activity when a monkey observed an experimenter reaching to grasp an object. This same neural population was not active when the monkeys observed an experimenter performing a reaching and grasping movement in the absence of the object. The critical condition, however, was when the experimenter reached to grasp an object that was hidden behind a screen so that the actual grasping action and the object was hidden from the monkey. In this condition, the neurons showed similar activity to the condition where the object was not occluded, but this occurred only when the monkey knew (had been shown) that an object was located behind the screen. When the monkey knew (had been shown) that no object was behind the screen, no increase in activity was observed for that neural population. Importantly, the kinematics of the action performed by the experimenter did not differ between the conditions; thus, the differences in motor activation in the monkey's brain cannot be explained by sensitivity to kinematics in the mirror-neuron system.

These results suggest that mirror neurons do not (or do not only) code for the observed actions themselves. Rather, their activity is decoupled from the observed action and their activity instead represents some higher-level property. Indeed, the only thing that differed between the two instances of hidden grasps was the monkey's knowledge of the action's context (that is, whether the action was directed at an object). Therefore, this difference, and a difference in the intended goal of the action, must be responsible for the observed differences in mirror-neuron system activation.

Similar sensitivity to action context has also been observed in the mirror-neuron system of humans. Iacoboni et al. (2005) used fMRI to measure brain activity while participants observed either actions taking place within a context, the context alone, or the action alone. Two action contexts were employed that implied different intentions for the observed actions. The contexts were a "drinking" context, where a cup was grasped with the intention of drinking from it, and a "cleaning" context, where a cup was grasped with the intention of taking it away for cleaning. The results showed that activity in motor regions 
thought to contain mirror neurons differed depending on which intention was present in the video clip. That is, as with the monkey studies performed by Umiltà et al. (2001), Iacoboni et al. (2005) found that the mirror-neuron system was sensitive to the observer's knowledge about the likely intention behind an observed action.

Taken together, these results show that when the observer knows the goal of an observed action, the motor system of the observer is engaged to generate the motor command for the action that might be employed to realise that goal. Put another way, when the monkey observes reaching, and the monkey knows that the goal is to grasp an object, the neural population responsible for grasping in the motor regions of the monkey's brain become active, even if the monkey doesn't actually observe grasping. Or, in the language of action control, the mirror-neuron system operates as an inverse model for the goals and actions of other people by taking the goal state of the observed agent (e.g., the desire to grasp an object) and reconstructing the motor command used for realising that goal (e.g., the motor command for grasping). ${ }^{2}$

The evidence just cited shows that observers' motor systems are sensitive to the goals of observed agents. But might observers' action planning and execution be similarity affected by the goals they assign to other agents? Recent evidence, using the automatic imitation paradigm, suggests that this is indeed the case. Automatic imitation, which has been used as a behavioural index of mirror neuron function (Longo, Kosobud, \& Bertenthal, 2008), is the phenomenon whereby participants are quicker to produce an action in response to a stimulus when that stimulus also depicts the to-be-produced action. For example, Brass, Bekkering, Wohlschläger, and Prinz (2000) presented participants with hand stimuli that contained a response-relevant stimulus dimension (a number) and a response-irrelevant stimulus dimension (a raised finger). Participants were asked to re-

\footnotetext{
${ }^{2}$ Note that these interpretations are the opposite of those usually presented in the literature. Many theorists suggest that the mirror-neuron system is used for recovering the goal of an observed action (e.g., see Gallese \& Goldman, 1998; Iacoboni et al., 2005; Rizzolatti \& Fabbri-Destro, 2008), while we, and others (e.g., Csibra, 2008; Jacob, 2008), argue that the activity of the mirror-neuron system is, in part, dependent on already having a goal representation for the observed action.
} 


\section{From action control to joint action}

spond to the number by raising their index finger in response to the number 1 and their middle finger in response to the number 2, while ignoring which finger was raised in the stimulus. Relative to a baseline condition, where the stimulus did not show a raised finger, participants' responses to the number were quicker when they were required to respond by raising the same finger that was raised in the stimulus (congruent condition) and slower when they were required to raise the other finger (incongruent condition).

Importantly for our purposes, automatic imitation tasks have been shown to be susceptible to the beliefs that the participant has about the intentions behind the observed actions. For example, Liepelt, von Cramon, and Brass (2008, Experiment 1), adapted the paradigm used by Brass et al. (2000) to examine the influence of inferred intentions on automatic imitation. They examined automatic imitation under different conditions that were designed to allow observers to infer different goals for the observed movements, but while holding the kinematics of the observed movements constant. The critical comparison was between the attempted movement condition (AMC) and the micromovement condition $(\mathrm{MMC})$. In the $\mathrm{AMC}$, the stimuli contained a hand with restrained fingers so that the fingers could not be fully raised. While in the $M M C$, the hand performed the same small movement but with the restraints absent. In the AMC, observers would be able to infer that the hand had attempted to fully raise the finger; that is, to produce the same movements that the participant was asked to produce in response to the symbolic cues. In the MMC, observers would be inclined to infer that the hand had not attempted to fully raise the finger, and that it had the goal of producing a different set of movements to the movements produced by the participants. The results showed that when the stimuli had the same set of goals as the participant (the goal of fully raising the fingers), responses were quicker when the particular goal of the stimulus matched the goal of the participant (congruent) relative to the case where the goal was not matched (incongruent). In contrast, when the stimulus performed movements with a different set of goals the observed movement did not modulate automatic imitation. This suggests that, rather than being driven solely by movement congruency, automatic imitation is also modulated 
by intention congruency.

A follow up experiment found that automatic imitation could also be modulated by the participant's beliefs about whether the observed actions were intentionally produced (Liepelt et al., 2008, Experiment 4), or their beliefs about whether the actions were produced by an intentional agent (Liepelt \& Brass, 2010). Taken together, these results suggest that rather than being driven by the observed action, the action representation formed in these tasks is decoupled from on-line input and is partly driven by the beliefs ascribed to the observed agent.

Work using a stimulus-response compatibility task, known as the Simon task, has also been used to study goal representations. By using two versions of the Simon task, one where participants act alone and one where participants act with another person, it is possible to examine whether individuals take into account the task or subgoals of their co-actors and represent them in a manner that is equivalent to how they represent their own subgoals. In particular, it is possible to examine whether participants form a representation of their co-actors' subgoals and whether these subgoal representations have functional consequences that are similar to individual subgoal representations. This we refer to as the task co-representation account (following Wenke et al., 2011) of the joint Simon effect, and below we outline the evidence that supports this account. Following this, we outline an alternative account, known as the actor co-representation account that has also been suggested for the findings from the joint Simon effect (Wenke et al., 2011). The actor co-representation account argues that the content of representation formed by an actor during joint tasks is not the subgoal of their co-actor. Rather, this account suggests that actors only form a representation that specifies that there is a co-actor responsible for the other half of the task. It should be noted that these two accounts are not mutually exclusive. Indeed, they may operate in different tasks and within the same task at different times. Therefore, we outline evidence that suggests that, at least in some instances, task co-representation does occur. In the final part of this section, we will also outline an account of the joint Simon effect that does not rely on co-representation but rather 


\section{From action control to joint action}

suggests that the results can be explained by the presence of another individual acting as a reference for spatially coding responses (the spatial coding account), and we will outline why we do not find this account compelling. However, we first turn our attention to the nature of the effect.

In the individual version of the Simon task, the participant is asked to make a response to one feature of a stimulus (e.g., colour or pitch) while ignoring an irrelevant spatial dimension (e.g., location). A simple example might require a participant to press a button with their right hand when they see a blue stimulus and another button with their left hand when they see a red stimulus. The typical finding is that when participants are asked to respond to a relevant stimulus feature while ignoring an irrelevant spatial feature of a stimulus, the spatial feature facilitates the response when it is spatially compatible with the response. For example, a response to a stimulus with the right hand might be facilitated when the stimulus is presented to the participant's right (e.g., Simon, 1969). The standard finding can be explained from a common-coding perspective (Prinz, 1997; Hommel et al., 2001). On this view, the spatial nature of the response leads to processing the irrelevant spatial feature of the stimuli because the stimulus and the response share a common representational format. When a stimulus appears, if the spatial features of the stimulus overlap with the spatial features of the response, then that response is facilitated relative to the case where the features do not overlap. Typically, this effect is only seen when participants are required to make responses with a spatial dimension-for example, responding with the left hand to one stimulus and the right hand to another-and are not seen in, for example, go/no-go tasks where participants make a single response to only one stimulus and not to the other stimulus.

The setup for Sebanz et al.'s (2003) version of the Simon task included both individual and joint versions of the task. In the individual version, participants performed either the standard two-choice task or a go/no-go task. In the standard two-choice condition participants sat directly in front of a monitor displaying the stimulus (a hand) and responded to the colour of a ring on the finger with either a left or a right button-press. 
Participants were asked to ignore the direction that the finger was pointing. In the individual and joint go/no-go conditions participants sat to one side of the monitor and made a response to only one colour ring and no response to the other. In the individual go/no-go condition the participant acted alone, and in the joint go/no-go condition each participant performed one half of the standard two-choice task where the go stimulus for one participant acted as the no-go stimulus for the other. The results of the experiment showed a typical spatial compatibility effect for the individual two-choice condition with responses facilitated when the hand pointed in the direction that was compatible with the button-press (i.e., left button-press when the hand pointed left). No compatibility effect was observed for the individual go/no-go condition. The key condition was the joint go/no-go condition. Rather than mirroring the results of the individual go/no-go condition, the joint go/no-go condition mirrored the individual two-choice condition. That is, participants performed the joint go/no-go task as if they had two action possibilities available. Importantly, this was only the case when the pair (participant and co-actor) actually had two choices available, as the effect did not occur when the participant was paired with a non-co-acting individual.

While the results of Liepelt and colleagues, using the automatic imitation paradigm (e.g., Liepelt \& Brass, 2010; Liepelt et al., 2008), have shown that goal-driven action representations for conspecifics can be partly decoupled from visual input, results using the joint Simon task suggest that an even more radical decoupling is possible. That is, it has been shown that joint Simon effects can be created even when participants receive no input about their co-actor. For example, studies by Tsai, Kuo, Hung, and Tzeng (2008) have shown that on cooperative tasks the presence of a second individual is not even necessary-the belief that one is co-acting on a joint task is sufficient to produce the joint Simon effect. In their study, a joint Simon effect occurred when participants individually performed a go/no-go Simon task under the belief that they were co-acting with a hidden human agent.

It should be noted that different results were obtained by Welsh, Higgins, Ray, and 


\section{From action control to joint action}

Weeks (2007). Welsh et al. (2007) found that the joint Simon effect disappeared after the confederate left the room. The authors of this study suggest that action observation is necessary for the joint Simon effect because action observation activates the action representations that cause the joint Simon effect. An explanation for the discrepancy between the results of Welsh et al. (2007) and Tsai et al. (2008) has been proposed by Vlainic, Liepelt, Colzato, Prinz, and Hommel (2010). These authors suggest that the failure of Welsh et al. (2007) to find the joint Simon effect in the confederate-absent condition may be due to participants not holding a sufficiently strong belief that they were co-acting with another intentional agent. In Welsh et al. (2007), participants' initial belief that they were co-acting may have diminished over time when they were not repeatedly updated about the presence of a co-actor, as was the case for Tsai et al. (2008). Interestingly, Tsai et al. (2008) were able to extinguish the joint Simon effect by modifying participants' beliefs so that they believed they were co-acting with a computer rather than an intentional agent. Taken together, these results support the notion that actors represent the specifics of the subgoals, or tasks, of their co-actors in the same manner in which they represent their own subgoals. Thus, it is labelled the task co-representation account of the joint Simon effect.

The task co-representation account is not the only account put forward to explain the results from the joint Simon task. At least two other alternatives, namely an actor corepresentation account and a spatial coding account, have also been suggested. According to the actor co-representation account, rather than representing what the other agent must do, participants merely represent that another agent is responsible for the other half of the task. On this account, the joint Simon effect arises because of conflict in determining whose turn it is to respond (Wenke et al., 2011). According to the spatial coding account—preferred by Guagnano, Rusconi, and Umiltà (2010) and Dolk et al. (2011) — the effects in the joint Simon task might be explained by the co-actor acting as a spatial reference for the participant's responses resulting in the participant's singular response being coded as either "left" or "right". 
A complete treatment of these alternatives is outside of the scope of the current paper, but it should be noted that the findings of at least one study cannot easily be reconciled with either the actor co-representation account or the spatial coding account. Welsh (2009) employed a version of the joint Simon task that added crossed-hand conditions where participants crossed their hands into their co-actor's side of space. The task corepresentation account, outlined earlier, would predict that the Simon effect would track the location of the hand, as is found in the individual version of the Simon task (e.g., Brebner, Shephard, \& Cairney, 1972). However, both the actor co-representation account and the spatial coding account would predict that the Simon effect would track the location of the participant's body. The actor co-representation account would predict this because crossing hands would not change the relationship between the spatial location of the participant and the "turn" cue provided by the irrelevant spatial dimension of the stimulus. The spatial coding account would predict the same, because, regardless of the hand position the, spatial reference provided by the co-actor's body would not change. Welsh's (2009) results showed that the Simon effect tracked the location of the hand; therefore, these results are more consistent with the task co-representation account.

While any one of the accounts may be superior at explaining a particular set of data, no one account has been able to successfully explain all the data. This, therefore, suggests that the explanations may not be mutually exclusive, but that the various mechanisms they each suggest may be in play only in certain tasks and at certain times. This is especially the case for the two co-representation accounts, task co-representation and actor co-representation. To illustrate their preferred account of actor co-representation, Wenke et al. (2011) introduce the example of a table tennis game. Task co-representation, or representing the other agent's subgoal, implies that an agent represents what stroke (e.g., top spin or back spin) their opponent will employ on each turn. Actor co-representation, on the other hand, merely implies that an agent will represent that it is their co-actor's turn to hit the ball rather than their own turn. However, it seems plausible that agents might represent that it is the other's turn at some points and then, when more information be- 


\section{From action control to joint action}

comes available, also represent what stroke their opponent will employ.

Taken together, the results of studies on automatic imitation and joint task paradigms, such as the joint Simon task, suggest that agents are able to represent the subgoals of their co-actors in a manner that has consequences for their own action planning and execution. This suggests that the others' subgoals are represented in the agent's action system in a manner that is functionally equivalent (that is, in a manner that has similar causal consequences) to how agents represents their own subgoals.

\subsubsection{Action models in predicting observed actions}

In the preceding section, we detailed how representations that participants form about the goals of other agents can affect action planning and execution in much the same way as their own individual goals can affect action planning and execution. We have suggested that this occurs because the goals of others are represented in much the same way as individual goals. Once a co-actor's goals have been represented, how are their actions predicted? These goals can be fed into the inverse model that is used to plan one's own actions to predict the motor commands that would be used to realise this goal. When limited information is known-for example, only the goal—the commands predicted by the inverse model can be inaccurate because many actions can be employed to realise a particular goal. Without detailed knowledge about the constraints and capabilities of the co-actor's action system it is difficult to constrain the possibilities. One way to constrain the process of predicting the correct motor commands is by supplying additional information, such as details about the co-actor's current limb state or details about actions they might currently be performing. Csibra (2008) suggests that visual analysis of the movements and bodies of other agents may be a crucial step in reconstructing the motor codes driving their actions. If estimates of the motor codes, generated by the inverse model, can be constrained based on visual analysis they could be used to drive the forward model and generate predictions about the unfolding dynamics of the observed action, and any discrepancy between the predicted and observed states could then be used to refine these 
estimates. In addition, the output of the forward model could be used to anticipate the actions of one's co-actor, thus making it possible to plan and execute complementary actions in a timely manner. In the following section we will outline the empirical evidence that suggests that action models, such as inverse and forward models, are involved not only in action production but also in predicting observed actions.

Wilson and Knoblich (2005) have put forward an account of action prediction wherein they suggest that observers use their own action dynamics as a model, or an emulator, for the action dynamics of conspecifics. The output of this emulator can then serve as the basis for anticipatory action planning or for aiding perception of conspecifics in suboptimal conditions where vision may be occluded by objects or other agents. For example, the observer can use the emulator to infer the trajectory of partially occluded actions because the movements of the observer and the observed agent are subject to similar (but not identical) constraints.

Several lines of evidence have been used to support the idea that the same mechanisms recruited by action production are also recruited for action prediction. For example, neurophysiological evidence suggests that overlapping neural populations in motor regions, such as premotor cortex (for a review, see Rizzolatti \& Craighero, 2004) and the cerebellum (e.g., Calvo-Merino, Grézes, Glaser, Passingham, \& Haggard, 2006), are involved in both the execution and observation of actions. These premotor regions also exhibit a topographic organisation (Buccino et al., 2001) similar to that observed in the motor homunculus (see Penfield, 1954). There are, however, competing interpretations for the neurophysiological data (e.g., see Gallese, 2009, and Jacob, 2008, for competing views) and, therefore, direct behavioural evidence may be more compelling. This evidence comes from an experimental paradigm examining differences in action prediction accuracy when observers view recordings of actions that were either self-produced or produced by another person. The logic of this paradigm is that if action prediction involves emulating the observed action with the observer's action model, then a high degree of similarity between the action model of the predicted system and the predicting system (as is found in 


\section{From action control to joint action}

the self-prediction condition) should result in superior prediction accuracy relative to the case where the action model of the predicted system and predicting system are dissimilar (as is found in the other-prediction condition). This self-prediction advantage has been termed an authorship effect (Knoblich \& Flach, 2001), and it can be used as an index of action emulation.

Several experiments conducted by Knoblich and colleagues have employed this paradigm. For example, Knoblich and Flach (2001) examined the ability of participants to predict the landing position of a thrown dart from a recording of the action. Participants based their predictions on videos that depicted the throwing action up until the point of release, thus excluding any information about the dart's flight trajectory. Consistent with the emulator hypothesis, the results showed that participants were significantly more accurate at predicting the landing position of darts they had thrown themselves - that is, there was an authorship effect. Importantly, if action emulation is to be useful for joint action coordination, then the predictions generated by the emulator system must be available in advance of incoming sensory information. One method to test how rapidly these predictions are available is to employ a synchronisation task, because this places a time constraint on prediction. As with the dart throwing experiment discussed above, it is possible to examine whether observers use their own action model during action synchronisation by measuring synchronisation accuracy for self-produced actions compared with other-produced actions.

Flach, Knoblich, and Prinz (2003) examined synchronisation accuracy using dynamic pen traces. In their study, participants first drew zigzag patterns on a drawing tablet. At a later stage, participants were presented with dynamic displays of the zigzag traces. They were asked to synchronise a button-press with the peaks of the trace and synchronisation performance was measured. The results showed that timing error was reduced when participants were synchronising with self-generated patterns compared with othergenerated patterns. This suggests that information from the observer's action model is available rapidly enough to be useful for action planning. 
However, an unexpected finding in Flach et al.'s (2003) study was that the authorship effect only emerged in the later experimental trials, suggesting that the action emulation system takes time to come on-line. One explanation for this is that for the forward model to accurately predict the sensory consequences of an action, the model needs accurate information about the current state of the effectors producing the action and accurate information about the motor codes corresponding to that action. The stimuli used by Flach et al. (2003) did not contain any information about the current state of the limbs and, therefore, this information would need to be reconstructed from the sensory consequences (pen traces) of the action. This could be done if estimates are generated about the state of the effectors together with estimates about the current motor codes. These estimates could be fed into the forward model, and the discrepancy between the output of the forward model and the actual observed sensory consequences could be used to update the estimates. Once the correct motor codes and state variables have been learnt (or rather, links have been generated between the motor codes and the observed traces) these can be used with the forward model to generate predictions. This process would not alter the internal dynamics of the forward model and, therefore, observers would still use their own action model to generate the predictions; therefore, the authorship effect would be maintained in later trials.

One way to circumvent the problem of having to learn the links between sensory effects and actions is by using participants with particular motor expertise, such as musicians, who would already have learnt the appropriate mappings between actions and sensory effects. Keller, Knoblich, and Repp (2007) asked expert musicians to synchronise a piano performance with recordings of either self-produced or other-produced music. The results showed that pianists were more accurate when playing a piano duet with a recording of self-produced music relative to other-produced music. In contrast to Flach et al. (2003), the authorship effect was present in the first block and did not change in magnitude as the experiment progressed. These results are consistent with trained pianists having more direct access to the links between perceived sensory consequences and the actions required 


\section{From action control to joint action}

to produce them (e.g., see Drost, Rieger, Brass, Gunter, \& Prinz, 2005; Repp \& Knoblich, 2007).

While the results of Keller et al. (2007) suggest that the action prediction system may come on-line rapidly enough for it to plausibly be involved in joint action coordination, the use of auditory stimuli and motor experts raises a question about how readily these results can be generalised to typical cases of joint action where non-experts engage in visually-coordinated actions. Similarly, there is also a question about how readily the results of Flach et al. (2003) and Keller et al. (2007) can be generalised to cases of actually predicting actions because both of these studies employed paradigms where participants generated predictions about the effects, or consequences, of actions - that is, dart throwing and piano playing actions, respectively. We have taken an alternative approach, which does not rely on using motor experts, to circumvent the problem of reconstructing the motor codes and state variables by modifying the paradigm of Flach et al. (2003) so that participants were shown depictions of moving bodies (Chapter 2). During the movement phase, participants were asked to produce arm movements by pretending to draw zigzag patterns on an imaginary blackboard. These actions were recorded using motion capture. The motion capture data was then reconstructed as an animated character. During the test phase, participants were asked to synchronise a button-press with the arm movements of the character, and timing error was measured. As in Flach et al. (2003), enhanced synchronisation accuracy was observed for self-generated actions; however, this authorship effect was present from the first block. This is to be expected if visual analysis of the limbs and limb movement is used to reconstruct the motor codes used to produce the action. With more immediate access to the motor codes, this information did not need to be learnt during the course of the experiment.

A follow up study provided further support for the benefit of providing bodily information in the stimulus (Chapter 3 ). Unlike previous studies that examined the difference between self-synchronisation and other-synchronisation, the experiment reported in Chapter 3 required all participants to synchronise with other-produced actions and, in- 
stead, varied the type of information available in the stimulus. We found that participants were significantly more accurate at synchronising with an animated character (full information) than they were at synchronising with a single moving point-light (point information). Importantly, this difference in synchronisation accuracy could not be explained by the visual properties of the stimulus, such as visual complexity, because the synchronisation advantage for full information stimuli was restricted to those participants who had received pre-experiment motor experience with the mannequin's action by performing the action themselves. ${ }^{3}$

There was also another difference in task performance between the participants that had motor experience with the action they were observing and the participants that did not have this experience. The movements used for the synchronisation task were irregular, with the timing between the synchronisation points varying throughout the course of a single movement display. The results showed that only participants with relevant movement experience tended to respond to these subtle timing differences. Participants who had not performed the actions tended to respond to the global timing characteristics of the stimulus, such as average timing of the synchronisation points. One reason for this difference might be that participants without motor experience do not have an internal representation of the action onto which they can map the stimuli and, therefore, they might only simulate the general dynamics of the stimulus rather than the dynamics of the specific effector used to produce the movement (Schubotz, 2007). Without replicating the specific effector, their internal model may be less able to reproduce the subtle kinematic properties of the action. And, because they do not emulate the specifics of the limb and joint movement, but only the general dynamics, this information is not incorporated into their internal model. This interpretation is also supported by neurophysiological evidence that suggests that the degree to which an observer can map observed actions onto their motor system is dependent on their motor experience (Calvo-Merino, Glaser, Grézes, Passingham, \& Haggard, 2005; Calvo-Merino et al., 2006). Importantly, these re-

\footnotetext{
${ }^{3}$ Participants performed the pre-experiment movements with their eyes closed so as to limit visual experience with the action.
} 


\section{From action control to joint action}

sults show that when generating predictions about actions, observers may represent the actual specifics of the action in a part-by-part manner, at least for actions that are part of their repertoire.

Taken together, these studies show that when generating predictions about merely observed actions, observers' motor dynamics influence the accuracy of the predictions that they generate. This can be explained by common mechanisms for action production and action prediction. As sensorimotor prediction during internally generated (performed) action is performed by means of forward models, it seems likely that functionally equivalent forward models are involved during sensorimotor prediction for externally generated (observed) actions. Furthermore, by employing a prediction mechanism, such as that described above, it might be possible to predict the actions of other agents without the need to form elaborate representations of their goals. For example, rudimentary goal conjectures, or simply motor code conjectures, could be formed on the basis of visual analysis of the observed actions. Predictions could then be generated on the basis of these conjectures, and these conjectures could then be updated using the error in the prediction.

\subsubsection{Coordinating actions in individuals and groups}

From the evidence cited above, it is clear that common mechanisms underlie action control and action prediction. Furthermore, the mechanisms that underlie action prediction can be directly recruited to assist joint action control in time-critical situations by allowing individuals to predict or anticipate the actions, and action-effects, of their co-actors. This has obvious applications for joint action. For many types of joint action, understanding the goals of one's co-actors or representing a joint goal is not sufficient for successfully engaging in the joint action. For example, in ensemble music and dance performance actions need to be coordinated with a high degree of temporal precision; this cannot be achieved through joint goal representation alone. Temporal coordination of action production between multiple co-actors is made possible by individuals co-representing their own subgoals with the subgoals of their co-actor and predicting self-generated and other- 
generated actions within a single system. That is, within a single action system, individuals are able to plan and execute their own actions while at the same time emulating the actions of their co-actors. When this type of integration is possible it should permit individuals to engage in joint activities in much the same way as individuals engage in individual activities. Indeed, experimental results from Knoblich and Jordan (2003) show that it is the case that the properties of action coordination between individuals show similarities to action coordination within individuals.

In the experiments of Knoblich and Jordan (2003), participants were given the task of controlling a circular ring (tracker) on a computer monitor so that it tracked the movement of a smaller dot (target). The target moved horizontally across the screen and changed direction at the edges. Two response buttons were used to control the speed of the tracker. The left response button increased the tracker's speed when the tracker was moving towards the left of the screen and decreased the tracker's speed when it was moving towards the right of the screen; the right response button had the opposite effect. In order to change the direction of movement the tracker first had to come to a complete stop.

Participants performed the tracking task either individually or in pairs with each participant controlling one button. The results showed that groups were able to learn to perform the task as well as individuals and in the same manner as individuals. However, this only occurred when groups were given feedback about the actions of their partner. It was suggested that without feedback participants were unable to distinguish the contribution of each participant to the movement of the tracker. But when feedback was presented, this allowed participants to emulate the actions and action effects of their partner along with representing their own actions and action effects (Knoblich \& Sebanz, 2008). This parallel emulation allowed them to construct a joint action representation equivalent to an individual action representation thus permitting joint action coordination that was functionally equivalent to individual action coordination.

When engaged in individual actions such as juggling, individuals are required to coordi- 


\section{From action control to joint action}

nate the actions of two hands. Being able to predict how one's own actions will influence the juggling balls has obvious benefits when trying to plan and coordinate the actions of the two hands (Wolfensteller, 2009). Juggling is by no means easy, but it has the advantage that the actions and action goals are represented in a single system and predictions can be generated by a single motor system designed for that task. The case of coordinating the actions of two individuals in time-critical situations seems far more difficult. However, the framework we have presented here suggests that the gulf between the nature of the mechanisms involved in the individual case and the joint case is not so large. As shown in Section 5.4.1, when engaged in joint actions individuals represent the tasks of their co-actors in a manner that is functionally equivalent to the way they represent their own tasks. Furthermore, as shown by the studies on action prediction (Section 5.4.2), individuals predict the actions and action-effects of their co-actors using the same mechanisms they use to predict their own actions and action effects. And, as the experiments of Knoblich and Jordan (2003) demonstrate, individuals engaged in joint tasks construct joint action models that are functionally equivalent to individual action models, and thus the system of individuals engaged in a joint task is able to coordinate action execution in the same way that an individual coordinates action execution in an individual task.

\subsection{Phenomenology of joint action}

The similarities between action coordination in the joint case and in the individual case may have further implications. The predictive mechanisms involved in individual action control have been used to explain the phenomenology of individual action (e.g., Frith et al., 2000; Pacherie, 2008). If, as we have argued, similar predictive mechanisms also underlie joint action control, then this raises the possibility that these predictive mechanisms might also help explain the phenomenology of joint action.

Frith et al. (2000) have used the model of action control developed by Wolpert (1997) to explain the sense of agency, or the sense of oneself being the cause of an action. In its simple form, this model, known as the comparator model, states that a sense of agency 
is derived by comparing sensory predictions generated by the forward action model with actual sensory feedback. If the predicted sensory feedback matches actual sensory feedback, then the action is perceived as self-produced-that is, a sense of agency is felt over the action. When the movement is not intended (for example, when it is caused by an external force), the initial motor command that ordinarily drives the functioning of the forward model is missing and, as a result, no sensory feedback is predicted. When sensory feedback is perceived, as a result of the limb's movement, a discrepancy between the predicted feedback and the actual feedback occurs. This prevents a sense of agency being felt over the action. Only when the feedback is accurately predicted by the forward model, as in the case of intentional action, is a sense of agency felt.

Wegner and colleagues (Wegner et al., 2004; see also Wegner, 2002) have also argued for a role for prediction in generating feelings of agency; however, their account does not specifically rely on the motor prediction found in the comparator model. According to their account, feelings of agency arise when a thought appears prior to an action that is consistent with the action and when there is no salient alternative cause for the action. While there are important differences between their account and the comparator model, they do share the notion that being able to predict actions, or action effects, plays an important part in feelings of agency.

\subsubsection{Sense of agency over observed actions}

Although it may seem that the sense of agency is always reliable in healthy people, this is in fact not the case and it has instead been shown that the sense of agency is subject to a number of illusions (Wegner, 2002). One example of the malleability of agency can be demonstrated in experiments that employ the helping hands paradigm. These experiments show that it is possible for people to feel a sense of agency over actions they are merely observing and not in fact performing. To create this illusion, Wegner et al. (2004) had participants stand in front of a mirror while another person (the hand helper) stood behind them, hidden from view, with their arms extended forward and positioned on 


\section{From action control to joint action}

either side of the participant as if they were the participant's own arms. A series of instructions to perform simple movements (e.g., "wave hello with your right hand") were then given to the hand helper alone or to both the participant and the hand helper. The instructions given to the participant could either be the same as those given to the hand helper or they could be different. Once the movements had been completed, participants were asked a series of questions including questions designed to probe their feelings of agency over the hand helper's actions (e.g., "How much control did you feel that you had over the arms' movements?").

The results showed that participants reported a sense of agency over the movement of the hand helpers' hands when they heard movement instructions that were consistent with the movement of the hand helpers' hands. (It should be noted that a sense of agency is not all-or-none but, as these experiments suggest, is graded). This effect persisted even when participants made simultaneous subtle movements of their own hands in a manner inconsistent with the movement of the hand helpers' hands. As with the comparator account of agency, Wegner et al.'s (2004) explanation of these data similarly rests on the idea of prediction. Wegner et al. (2004) suggest that the erroneous feeling of agency arises because when participants are able to hear the movement instructions they are able to anticipate the movement of the hands.

The results of the helping hands paradigm have not ordinarily been interpreted from a forward-modelling perspective. Both proponents (e.g., Carruthers, 2010) and critics (e.g., Synofzik, Vosgerau, \& Newen, 2009) of the comparator model have claimed that the helping hands paradigm poses a problem for the comparator model because it is assumed that there is no motor prediction with which to compare actual sensory feedback. However, data from Aziz-Zadeh, Wilson, Rizzolatti, and Iacoboni (2006) suggests a way in which the comparator model might be able to account for this phenomenon. Their data show that reading phrases describing actions (e.g., "biting the peach", "grasping the scissors") is sufficient to activate premotor regions that form part of a network that has been implicated in action prediction (Csibra, 2008; Wilson \& Knoblich, 2005). Therefore, it is plausible that 
hearing action phrases may be sufficient for generating an emulated motor command and motor prediction. The helping hands paradigm might also be considered an instance of a joint task between the participant and the hand helper. As argued in Section 5.4, when engaged in a joint task, actors generate predictions the about actions of their co-actors by emulating their actions. A match between these predictions and the actually occurring actions might thus contribute the illusory feelings of agency reported in the helping hands task. Indeed, Wegner et al. (2004, p. 847) state: “We could also feel slight pangs of authorship when we see a cartoon figure run off a cliff and plummet to the valley below... because of our ability to anticipate the action"-although in this case Wegner et al. (2004) do not mean motor prediction; however, as shown in Section 5.4, motor prediction is the means by which we achieve the ability to predict the actions of others in joint tasks.

While one might consider the helping hands task as an instance of a joint task it does not seem to match exactly with what might commonly be construed as a joint task. This raises the question of whether there is any evidence of disruptions, illusions, or alterations in the sense of agency occurring in more genuinely joint tasks. In the section that follows we turn our attention to this question.

\subsubsection{Disruptions in the sense of agency during joint action}

The use of predictive mechanisms during both action control and joint action coordination (as argued in Section 5.4) may also have impacts on the sense of agency during certain instances of joint action, as well as the phenomenology of joint action more generally. The experiences reported by people engaged in joint action range from feelings of group flow (Sawyer, 2006) at one end of the spectrum to feelings of loss of self and "oneness" at the other (Hanna, 1977; McNeill, 1995). In the section that follows, we argue that these experiences arise out of the ability to predict the actions of others just as the feeling of individual agency, and the phenomenology of individual action, arises out of our ability to predict our own actions.

Engaging in synchronised imitative action may be an especially powerful means of evok- 


\section{From action control to joint action}

ing subtle disruptions in the sense of agency by spreading the sense of agency beyond the individual so that the individual feels a sense of agency over the collective action. Reports from synchronised military marching drills describe such an experience. For example, the historian McNeill (1995) notes that during his own experience of military marching drills he felt "a strange sense of personal enlargement... becoming bigger than life, thanks to participation in collective ritual" (p. 2). Similar experiences can also occur during synchronised dancing — for example, Hanna (1977, p. 119) reports "boundary loss, [and] the submergence of the self in a flow" during African dance rituals. McNeill (1995) notes that Hanna's (1977) reports of "boundary loss" and feelings of "oneness" and his experiences during marching drills amount to the same thing: "a blurring of self-awareness and the heightening of fellow-feeling with all" (p. 8) engaged in the collective action, and a shifting of the sense of self from the individual to the group. Synchronised action, as argued in Section 5.4.2, may rely on the ability to predict the actions of others and, therefore, successfully predicting the actions of the group, and the sensory consequences of those actions may mediate the experiences described above.

Sebanz et al. (2006) and Hove (2008) have also suggested that joint action can lead to disruptions in the feeling of agency. For instance, while prediction of action-effects ordinarily serves as a cue for who is the agent responsible for an action, Sebanz et al. (2006) suggest that this cue may not be robust in instances of joint action where similar predicted action-effects could have been caused by oneself or one's co-actor. In turn, Hove (2008) has proposed that during synchronised action, difficulty in distinguishing self-produced from other-produced actions can lead to a blurring in the distinction between self and other. This blurring of the self-other distinction parallels Hanna's (1977) "boundary loss" and McNeill's (1995) "blurring of self-awareness".

Hove's (2008) argument builds on the claim that accurate prediction of the sensory consequences of an action can be used as a cue for attributing our own actions to ourselves. However, if, when observing actions, we use functionally equivalent action models to predict the actions of our co-actors, then action attribution based on prediction 
accuracy might not be effective. A second cue for action attribution may be motor output as this is ordinarily inhibited during action observation. However, when engaged in synchronous action, where individuals mimic each other's action in time and form, lack of motor output is also an ineffective cue. In these situations, Hove (2008) suggests that the distinction between self and other can dissolve, in a kind of blurring that might lead to enhanced social bonding. The blurring of the self-other distinction might also have consequences beyond the spreading of agency, and it might also be a profound bonding experience. Examining this idea further, Hove and Risen (2009) asked participants to tap either synchronously or asynchronously with an experimenter. An affiliation measure was then recorded by asking participants how likable they found the experimenter. Affiliation scores were reliably modulated by the degree of synchronisation between the participant and the experimenter, suggesting that engaging in synchronised behaviour facilitates social bonds. This facilitation of social bonds is reminiscent of McNeill's (1995) reported "heightening of fellow-feeling" during marching drills. Furthermore, that affiliation scores can be modulated by the simple task used by Hove and Risen (2009) suggests that fairly low-level mechanisms at least partly underlie this phenomenon.

Hove and Risen (2009) suggest that their results may help to explain the social bonding effect of joint music performance. Freeman (2000) has argued that music in preliterate cultures likely took the form of synchronised social actions comprised of rhythmic chanting, clapping, and drumming. Furthermore, highly constrained, rhythmically synchronised actions (such as group chanting) are common in religious ceremonies even today. Freeman (2000) has suggested that music evolved as a technology for group formation, and Hove and Risen (2009) suggest that blurring of the self-other distinction may be the means by which this occurs.

This subtle blurring of the self-other distinction might not only occur during instances of imitative action. For example, experiments by Wegner et al. (2004, see Section 5.5.1) show that people can experience a sense of agency over observed actions even when they simultaneously produce non-imitative actions. This suggests that predictive action 


\section{From action control to joint action}

plans, rather than imitative actions, might be responsible for the spreading of the sense of agency during joint action. Therefore, these effects might also occur during ensemble music performance or joint sport performance, as these tasks similarly engage predictive mechanisms. Sebanz et al. (2006) suggest that during ensemble music performance a spreading of agency might actually be preferred and musicians might "strive to experience a sense of agency that transcends individual boundaries" (p. 75).

The spreading of the sense of agency might also be accompanied with an experience that Sawyer (2006) has termed group flow, and this might similarly be mediated by predictive mechanisms. The term group flow is used to describe the state of a group when they perform as a single unit. When experiencing group flow "each of the group members can even feel as if they are able to anticipate what their fellow performers will do before they do it" (Sawyer, 2006, p. 158). That is, group flow describes instances of transparent coordination. Berliner (1994) and Monson (1996) provide many firsthand accounts from ensemble jazz musicians that illustrate the experience of group flow, which jazz musicians describe as "striking a groove" or simply "groove". Berliner (1994) states, "the highest points of improvisation occur when the group members strike a groove together" (p. 388). Striking a groove allows "everything to come together in complete accord" (Harold Ousley, quoted in Berliner, 1994, p. 349).

Jazz musicians often practice extensively in order to develop precise timing, and work hard to ensure that their playing is appropriately synchronised (Berliner, 1994). Keller (2008; see also Keller et al., 2007) has suggested that the ability to engage in rhythmically coordinated music performance may be partially mediated by predictive action models. Extensive practise may allow fine-tuning of these predictive models so that musicians are able to accurately predict the actions of their co-performers (see Cross, Hamilton, Kraemer, Kelly, \& Grafton, 2009, for an example of training effects on action emulation for dance). Through this training, musicians may learn to associate particular emulated motor commands with particular sensory consequences in much the same way as individuals learn to associate particular sensory consequences with their own motor commands (e.g., 
see Section 5.3). Indeed, the musicians interviewed by Berliner (1994) often report the ability to accurately predict the actions of the other performers. For example, George Duvivier (quoted in Berliner, 1994, p. 390) reports that, "I've experienced times in which it was almost like I've been able to read a soloist's mind. I'll play a phrase, like a descending passage, at the same time he does". Some performers even report feelings of disembodiment reminiscent of Hanna's (1977) “boundary loss" and McNeill’s (1995) "blurring of self-awareness": For example, Leroy Williams (quoted in Berliner, 1994, p. 413) recalls, "the first time [he] experienced that floating, out-of-the-body feeling" while Michael Carvin compared the feeling to being in a trance and "being out of yourself" (quoted in Monson, 1996, p. 68). We suggest that these feelings of disembodiment and group flow result in part because the action systems of performers in these ensembles are finely tuned to those of their co-actors. And they are engaged in predicting the actions of, and the sensory effects produced by, their co-actors and the combined sensory effects produced by the ensemble's actions. In attempting to synchronise their musical performance they predict how their fellow musicians will perform, and the accuracy of these predictions, in part, leads to experiences of group flow and a spreading of the sense of agency. Furthermore, as Hove and Risen's (2009) experiment suggests, "striking a groove" or the experience of group flow can also be a profound bonding experience. For example, Chuck Israels reports "[y]ou play every beat in complete rhythmic unison... if it's working, it brings you very close... The relationship is very intimate" (quoted in Berliner, 1994, pp. 349-350).

Successfully playing music together requires musicians to not only predict and anticipate the actions of their fellow musicians, but to also plan and execute their own actions in response to these predictions in such a way so that the group is able to bring about their desired goal (the successful performance of the music). We have argued that this process of interpersonal coordination relies on the same mechanisms responsible for intrapersonal coordination. That is, coordinating action between two limbs relies on the same mechanisms responsible for coordinating action between two people, albeit with an emulated subgoal and an emulated motor command for the co-actor. In the case of 


\section{From action control to joint action}

intrapersonal coordination, a veridical sense of agency is felt over the consequences of the inter-limb coordination when the consequences of the inter-limb coordination are accurately predicted. Similarly, the framework presented here suggests that musicians, when striking a groove or experiencing feelings of group flow, may similarly feel a sense of agency that transcends the self and that manifests as a sense of agency over the product of the interaction (i.e., the music as a whole). Due to the conditions needed to create these experiences - that is, the requirement that the consequences of the interaction are accurately predicted-it is likely that it is difficult to elicit these experiences. Furthermore, a spreading of the sense of agency and feelings of group flow do not describe a unitary phenomenon; however, we argue that the predictive mechanisms described in Section 5.4.2 may play an important role in creating both experiences. Even in experienced musicians, where predictive mechanisms may be especially finely tuned due to the fact that joint music performance critically depends on their optimal functioning, these experiences may be rare, which may further add to their value as a highly prized aesthetic ideal (Monson, 1996).

\subsection{Conclusions}

We have outlined a framework for understanding joint action control that is built on a foundation of individual action control. Individual action control relies on inverse and forward models in order to transform goals, first, into control commands and then to generate predictions about how the action will unfold or the sensory effects that will arise. We suggest that this system can be taken off-line in order to transform a co-actor's goals into action and sensory predictions. Ultimately, the similarities between individual action and joint action, as conceived by our framework, provide hints for understanding the phenomenology of joint action. While explanations of the phenomenology of group performance are still speculative, the model proposed here suggests that the mechanisms that give rise to the phenomenology of acting alone may also prove useful for an account of the phenomenology of acting together. Further empirical investigations are still needed 
in order to test the validity of this claim; however, the framework presented here can serve as a guide for future investigations not only in the domains of cognitive neuroscience and psychology but also in phenomenology of joint performance, which up until now has been a relatively unexplored area. An adequate taxonomy of the variety of experiences that occur during joint action will also be needed so that empirical work can pick out the correct explanatory targets. Future work will also need to address in more detail how control commands are reconstructed during joint action, and how prediction mechanisms are fine-tuned for joint action and group flow. An understanding of the neural mechanisms involved in body perception, biological motion perception, and perception of action effects may be particularly suited to answering these questions. An understanding of these mechanisms may elucidate the process by which perceived actions and actions effects can be transformed into motor representations in the absence of motor production. However, we believe that there is now a strong case to be made that a future understanding of joint action will be built on a foundation of individual action control.

\subsection{References}

Aziz-Zadeh, L., Wilson, S. M., Rizzolatti, G., \& Iacoboni, M. (2006). Congruent embodied representations for visually presented actions and linguistic phrases describing actions. Current Biology, 16(18), 1818-1823.

Berliner, P. F. (1994). Thinking in jazz: the infinite art of improvisation. Chicago: University of Chicago Press.

Brass, M., Bekkering, H., Wohlschläger, A., \& Prinz, W. (2000). Compatibility between observed and executed finger movements: comparing symbolic, spatial, and imitative cues. Brain and Cognition, 44(2), 124-143.

Bratman, M. E. (1993). Shared intention. Ethics, 104(1), 97-113.

Brebner, J., Shephard, M., \& Cairney, P. (1972). Spatial relationships and S-R compatibility. Acta Psychologica, 36(1), 1-15. 
Buccino, G., Binkofski, F., Fink, G. R., Fadiga, L., Fogassi, L., Gallese, V., .. Freund, H. J. (2001). Action observation activates premotor and parietal areas in a somatotopic manner: an fMRI study. European Journal of Neuroscience, 13(2), 400-404.

Calvo-Merino, B., Glaser, D. E., Grézes, J., Passingham, R. E., \& Haggard, P. (2005). Action observation and acquired motor skills: an fMRI study with expert dancers. Cerebral Cortex, 15(8), 1243-1249.

Calvo-Merino, B., Grézes, J., Glaser, D., Passingham, R., \& Haggard, P. (2006). Seeing or doing? influence of visual and motor familiarity in action observation. Current Biology, 16(19), 1905-1910.

Carruthers, G. (2010). A problem for wegner and colleagues' model of the sense of agency. Phenomenology and the Cognitive Sciences.

Clark, A. (2003). Natural-born cyborgs : minds, technologies, and the future of human intelligence. New York: Oxford University Press.

Cross, E. S., Hamilton, F. d. C., Kraemer, D. J. M., Kelly, W. M., \& Grafton, S. T. (2009). Dissociable substrates for body motion and physical experience in the human action observation network. European Journal of Neuroscience, 30, 1383-1392.

Csibra, G. (2008). Action mirroring and action understanding: an alternative account. In P. Haggard, Y. Rossetti \& M. Kawato (Eds.), Sensorimotor foundations of higher cognition (pp. 435-459). New York: Oxford University Press.

Dolk, T., Hommel, B., Colzato, L. S., Schütz-Bosbach, S., Prinz, W., \& Liepelt, R. (2011). How "social" is the social simon effect? Frontiers in Psychology, 2.

Dreyfus, H. L. (1991). Being-in-the-world: a commentary on Heidegger's Being and time, division I. Cambridge MA: MIT Press.

Drost, U. C., Rieger, M., Brass, M., Gunter, T. C., \& Prinz, W. (2005). Action-effect coupling in pianists. Psychological Research, 69(4), 233-241.

Elsner, B., \& Hommel, B. (2001). Effect anticipation and action control. Journal of Experimental Psychology: Human Perception and Performance, 27(1), 229-240. 
Flach, R., Knoblich, G., \& Prinz, W. (2003). Off-line authorship effects in action perception. Brain and Cognition, 53(3), 503-513.

Freeman, W. (2000). A neurobiological role of music in social bonding. In N. L. Wallin, B. Merker \& S. Brown (Eds.), The origins of music (pp. 411-424). Cambridge MA: MIT Press.

Frith, C. D., Blakemore, S. J., \& Wolpert, D. M. (2000). Abnormalities in the awareness and control of action. Philosophical Transactions of the Royal Society B: Biological Sciences, 355, 1771-1788.

Gallese, V. (2009). Motor abstraction: a neuroscientific account of how action goals and intentions are mapped and understood. Psychological Research, 73(4), 486-498.

Gallese, V., \& Goldman, A. (1998). Mirror neurons and the simulation theory of mindreading. Trends in Cognitive Sciences, 2(12), 493-501.

Golnaraghi, M., \& Kuo, B. C. (2010). Automatic control systems (9th ed.). Hoboken NJ: Wiley.

Grush, R. (1997). The architecture of representation. Philosophical Psychology, 10(1), 523.

Grush, R. (2004). The emulation theory of representation: motor control, imagery, and perception. Behavioral and Brain Sciences, 27(3), 377-396.

Guagnano, D., Rusconi, E., \& Umiltà, C. A. (2010). Sharing a task or sharing space? on the effect of the confederate in action coding in a detection task. Cognition, 114(3), $348-355$

Hanna, J. L. (1977). African dance and the warrior tradition. Journal of Asian and African Studies, 12(1-4), 111-133.

Hommel, B. (2003). Planning and representing intentional action. The Scientific World, 3, 593-608.

Hommel, B. (2009). Action control according to TEC (theory of event coding). Psychological Research, 73(4), 512-526. 
Hommel, B., Müsseler, J., Aschersleben, G., \& Prinz, W. (2001). The theory of event coding: a framework for perception and action planning. Behavioral and Brain Sciences, 24(5), 849-878.

Hove, M. J. (2008). Shared circuits, shared time, and interpersonal synchrony. Behavioral and Brain Sciences, 31(1), 29-30.

Hove, M. J., \& Risen, J. L. (2009). It's all in the timing: interpersonal synchrony increases affiliation. Social Cognition, 27(6), 949-960.

Iacoboni, M., Molnar-Szakacs, I., Gallese, V., Buccino, G., Mazziotta, J. C., \& Rizzolatti, G. (2005). Grasping the intentions of others with one's own mirror neuron system. PLoS Biology, 3(3), e79.

Jacob, P. (2008). What do mirror neurons contribute to human social cognition? Mind \& Language, 23(2), 190-223.

Keller, P. E. (2008). Joint action in music performance. In F. Morganti, A. Carassa \& G. Riva (Eds.), Enacting intersubjectivity: a cognitive and social perspective on the study of interactions (pp. 205-221). Amsterdam: IOS Press.

Keller, P. E., Knoblich, G., \& Repp, B. H. (2007). Pianists duet better when they play with themselves: on the possible role of action simulation in synchronization. Consciousness and Cognition, 16(1), 102-111.

Knoblich, G., \& Flach, R. (2001). Predicting the effects of actions: interactions of perception and action. Psychological Science, 12(6), 467-472.

Knoblich, G., \& Jordan, J. S. (2003). Action coordination in groups and individuals: learning anticipatory control. Journal of Experimental Psychology: Learning, Memory, and Cognition, 29(5), 1006-1016.

Knoblich, G., \& Sebanz, N. (2008). Evolving intentions for social interaction: from entrainment to joint action. Philosophical Transactions of the Royal Society B: Biological Sciences, 363(1499), 2021-2031.

Liepelt, R., \& Brass, M. (2010). Top-down modulation of motor priming by belief about animacy. Experimental Psychology, 57, 221-227. 
Liepelt, R., von Cramon, D. Y., \& Brass, M. (2008). What is matched in direct matching? intention attribution modulates motor priming. Journal of Experimental Psychology: Human Perception and Performance, 34, 578-591.

Longo, M. R., Kosobud, A., \& Bertenthal, B. I. (2008). Automatic imitation of biomechanically possible and impossible actions: effects of priming movements versus goals. Journal of Experimental Psychology: Human Perception and Performance, 34(2), 489501.

McNeill, W. (1995). Keeping together in time : dance and drill in human history. Cambridge MA: Harvard University Press.

Miall, R. C., \& Reckess, G. Z. (2002). The cerebellum and the timing of coordinated eye and hand tracking. Brain and Cognition, 48(1), 212-226.

Miall, R. C., \& Wolpert, D. M. (1996). Forward models for physiological motor control. Neural Networks, 9(8), 1265-1279.

Monson, I. (1996). Saying something : jazz improvisation and interaction. Chicago: University of Chicago Press.

Pacherie, E. (2008). The phenomenology of action: a conceptual framework. Cognition, 107(1), 179-217.

Penfield, W. (1954). Mechanisms of voluntary movement. Brain, 77(1), 1-17.

Prinz, W. (1997). Perception and action planning. European Journal of Cognitive Psychology, 9(2), 129.

Repp, B. H., \& Knoblich, G. (2007). Action can affect auditory perception. Psychological Science, 18(1), 6-7.

Rizzolatti, G., \& Craighero, L. (2004). The mirror-neuron system. Annual Review of Neuroscience, 27(1), 169-192.

Rizzolatti, G., \& Fabbri-Destro, M. (2008). The mirror system and its role in social cognition. Current Opinion in Neurobiology, 18(2), 179-184. 
Rizzolatti, G., Fogassi, L., \& Gallese, V. (2001). Neurophysiological mechanisms underlying the understanding and imitation of action. Nature Reviews: Neuroscience, 2(9), $661-670$.

Sawyer, R. K. (2006). Group creativity: musical performance and collaboration. Psychology of Music, 34(2), 148-165.

Schubotz, R. I. (2007). Prediction of external events with our motor system: towards a new framework. Trends in Cognitive Sciences, 11(5), 211-218.

Searle, J. (1990). Collective intentions and actions. In P. R. Cohen, J. Morgan \& M. E. Pollack (Eds.), Intentions in communication (pp. 401-415). Cambridge MA: MIT Press.

Sebanz, N., Knoblich, G., \& Prinz, W. (2003). Representing others' actions: Just like one’s own? Cognition, 88(3), B11-B21.

Sebanz, N., Bekkering, H., \& Knoblich, G. (2006). Joint action: bodies and minds moving together. Trends in Cognitive Sciences, 10(2), 70-76.

Simon, J. R. (1969). Reactions toward the source of stimulation. Journal of Experimental Psychology, 81(1), 174-176.

Synofzik, M., Vosgerau, G., \& Newen, A. (2009). Reply to Carruthers. Consciousness and Cognition, 18(2), 521-523.

Tsai, C., Kuo, W., Hung, D. L., \& Tzeng, O. J. L. (2008). Action co-representation is tuned to other humans. Journal of Cognitive Neuroscience, 20(11), 2015-2024.

Umiltà, M. A., Kohler, E., Gallese, V., Fogassi, L., Fadiga, L., Keysers, C., \& Rizzolatti, G. (2001). I know what you are doing: a neurophysiological study. Neuron, 31(1), 155165.

Vlainic, E., Liepelt, R., Colzato, L. S., Prinz, W., \& Hommel, B. (2010). The virtual co-actor: the social Simon effect does not rely on online feedback from the other. Frontiers in Psychology, 1.

Wegner, D. M. (2002). The illusion of conscious will. Cambridge MA: MIT Press.

Wegner, D. M., Sparrow, B., \& Winerman, L. (2004). Vicarious agency: experiencing control over the movements of others. Journal of Personality, 86(6), 838-848. 
Welsh, T. N. (2009). When $1+1=1$ : the unification of independent actors revealed through joint Simon effects in crossed and uncrossed effector conditions. Human Movement Science, 28(6), 726-737.

Welsh, T. N., Higgins, L., Ray, M., \& Weeks, D. J. (2007). Seeing vs believing: is believing sufficient to activate the processes of response co-representation? Human Movement Science, 26(6), 853-866.

Wenke, D., Atmaca, S., Holländer, A., Liepelt, R., Baess, P., \& Prinz, W. (2011). What is shared in joint action? Issues of co-representation, response conflict, and agent identification. Review of Philosophy and Psychology, 2, 147-172.

Wilson, M., \& Knoblich, G. (2005). The case for motor involvement in perceiving conspecifics. Psychological Bulletin, 131(3), 460-473.

Witney, A. G. (2004). Internal models for bi-manual tasks. Human Movement Science, 23(5), 747-770.

Wolfensteller, U. (2009). Juggling with the brain—thought and action in the human motor system. In M. Raab, J. G. Johnson \& H. R. Heekeren (Eds.), Mind and motion: the bidirectional link between thought and action (pp. 289-301). Amsterdam: Elsevier.

Wolpert, D. M. (1997). Computational approaches to motor control. Trends in Cognitive Sciences, 1(6), 209-216.

Wolpert, D. M., \& Kawato, M. (1998). Multiple paired forward and inverse models for motor control. Neural Networks, 11(7-8), 1317-1329. 



\section{Part IV.}

Action synchronisation in other contexts 

CHAPTER 6

Music, action, and affect

Music is a powerful tool for generating and shaping emotional experiences. The emotional power of music operates through a number of distinct mechanisms. Listening to music can lead to emotional experiences through its ability to evoke memories or images, or by repeated association with emotional states. A piece of music may be reminiscent of a sad time in one's life and this episodic memory, in turn, may trigger feelings of sadness. The various sonic attributes may remind a listener of the sound of a waterfall, wind blowing through trees, or a vast and isolated landscape. These images and ideas may also trigger emotional associations. Music can also evoke emotions because of social, cultural or political associations. A national anthem can, for some, evoke powerful emotions of pride and joy no matter how tortured the rendition. Conversely, music that is beautifully composed and sensitively performed may, if used as propaganda in a racist campaign, evoke sinister associations and negative emotions. In all these examples, the music itself is not the primary object of the emotional experience. Emotional experiences are partly triggered by the music, but the memories, imagery, or associations are what actually drive the emotional experience. 
In this chapter, we outline a model that aims to explain how music, as the paradigm example of an embodied signal, can be the direct object of powerful emotional experiences. We will argue that the direct, unmediated emotional responses to music can be explained by viewing music listening as an embodied experience that engages sensory-motor processes. First, we illustrate the multi-modal nature of music by reviewing evidence that merely observing the actions that accompany music performance greatly influences our perception and interpretation of the acoustic dimension of music: the properties of the music that we hear. Second, we introduce a theoretical framework that views perception and action as inextricably linked, and that construes music as a unique type of multimodal behaviour specialised for engaging predictive sensory-motor processes in listeners. By this means, our perception of the qualities of music can directly guide the arousal of emotions. Moreover, we demonstrate that this framework allows for a unification of action-based models of emotion with expectancy-based models of musical emotions.

\subsection{Music and gesture}

Before the invention of the gramophone and the rise of the recording industry, music was almost always experienced as a multi-modal phenomenon containing auditory, visual, and kinesthetic dimensions. Moreover, before the rise of concert halls, music was rarely observed passively, but was actively experienced; music making was often social, collaborative, accompanied by dance, and had weak or absent boundaries between performer and audience (see Freeman, 2000, for a discussion of music as a technology for social bonding). Indeed, people are highly attuned to the human movements that are used to produce musical sounds. Movements such as banging on a drum or plucking a guitar string function not only to produce musical sounds; they provide a visual signal to others engaged in the musical activity. Visual signals, such as facial expressions and bodily movements (which we broadly call gesture), are integral to musical communication and they shape the way music is perceived, understood, and experienced. Research conducted over the past decade has rediscovered and confirmed the multi-modal, embodied nature 
of music experience: how visual and bodily dimensions of music (both on the part of the performer and the audience) inform and interact with the acoustic dimension of music, giving rise to complex psychological and social effects.

\subsection{The influence of gesture on music perception}

Groundbreaking research over 30 years ago illustrated that the facial movements that accompany speech have striking effects on how speech sounds are perceived. McGurk and Macdonald (1976) asked participants to listen to spoken syllables (such as [ga] or [ba]) and to report the syllable that they heard. Participants performed the task with correct identification of syllables in over $90 \%$ of trials. However, performance was dramatically altered when the acoustic signal for one spoken syllable was synchronised with the mouth movements used to produce a different spoken syllable. When the acoustic signal of [ba] was synchronised with a video of the mouth movements required to produce the syllable [ga], participants often reported hearing the syllable [da]. This illusory percept, known as the McGurk effect, was dependent on the presence of the visual signal: by closing their eyes, participants could once again accurately identify the vocalised syllable.

In a similar fashion, movements that accompany music performance influence the perception of musical structure. Thompson, Graham, and Russo (2005, Experiment 1 ) found that facial expressions and gestures that occur during music performance influence the perception of "musical dissonance". Musical dissonance occurs when a pitch or pitch combination is perceived to clash with (learnt) harmonic or tonal expectations, resulting in music that sounds discordant or in need of resolution (for a discussion of dissonance, see Huron, 2006, pp. 310-312; Thompson, 2009, pp. 48-50). It is differentiated from sensory dissonance-the sensation of beating and roughness associated with interactions among sound waves (amplitude fluctuations) and the inner ear's ability to resolve spectral components.

In order to examine the role of facial expressions and gestures on perceived musical dissonance, 20 excerpts from an audio-visual recording of B. B. King playing the blues 
were selected. Ten of these excerpts contained facial expressions that conveyed a sense of dissonance (dissonant set) while the other ten excerpts contained more neutral facial expressions (neutral set). The actual level of musical dissonance in the two sets of excerpts was similar, so the only systematic difference between sets was the type of facial expressions used. Facial expressions and gestures in the dissonant set included wincing of the eyes, shaking of the upper body, a grimace, and a rolling of the head in a back-swung position. Such expressions may have resulted from an increased (or feigned) difficulty of performing dissonant notes, but they may also be introduced to signify a performer's interpretation of musical structure. Participants were presented with both sets of excerpts under audio-visual and auditory-only conditions and judged the level of dissonance for each excerpt. Dissonance was explained to participants as "occurring when the music sounds discordant (i.e., conflicted or negative) and in need of some sort of resolution." (Thompson et al., 2005, p. 189). In the audio-visual condition, excerpts that contained dissonant facial expressions and gestures were rated as more dissonant musically than excerpts that contained neutral facial expressions. However, when those same passages were presented in the absence of facial expressions (audio-only condition), ratings of dissonance were no different in the two sets. That is, the inclusion of facial expressions and gesture was wholly responsible for the perceived dissonance of the excerpts. These results are consistent with the notion that visual information associated with musical performance can significantly influence how audiences perceive and encode consonance and dissonance.

If facial expressions during music performance can influence the way acoustic information is perceived, then it stands to reason that such facial expressions might influence the perception of musical structure. Thompson et al. (2005, Experiment 2) examined whether facial expressions used by singers could communicate to audience members the size of a melodic interval they were singing. Participants were asked to view (video-only) or listen to (audio-only) a trained musician sing four intervals that ranged in size between two and nine semitones. For both audio-only and video-only presentations, ratings of inter- 
val size by musically untrained participants were highly correlated with the actual size of intervals illustrating that musical structure is conveyed not only by the acoustic dimension of music, but also by visual signals arising from the facial expressions and gestures of musicians. Even more striking, the effect of facial expressions was evident even when acoustic information was present (Thompson et al., 2005, Experiment 3). For example, ratings of interval size were significantly higher if an auditory signal arising from a small melodic interval (two semitones) was paired with facial expressions associated with the production of a large melodic interval (nine semitones) than when it was paired with facial expressions associated with the production of a small melodic interval (two semitones).

Subsequent research was conducted to identify specific facial features associated with melodic interval size. Thompson and Russo (2007) asked participants to judge the size of a sung interval from silent video recordings. As with Thompson et al. (2005, Experiment 2 and 3), participants' ratings were strongly correlated with actual interval size. The authors also measured several types of facial movements in their stimuli. These included head and eyebrow displacement (measured as the maximum distance relative to start positions) and the size of mouth opening (measured as the maximum distance between the upper and lower lip). All movements measured were correlated with interval size suggesting that participants have access to multiple and partially redundant visual cues for extracting melodic interval size.

Musicians may produce these movements for a number of reasons. First, they may reflect production constraints: producing a large interval requires a large change in vocal cord tension, but the muscles used to implement this change in vocal tension might inadvertently recruit muscles that control facial features. Second, such movements may be an explicit attempt by musicians to communicate the size of a pitch interval. Third, facial movements may be an implicit form of communication built into conventional expressive actions and gestures among performers because of their success at enhancing musical communication and, hence, enjoyment. Fourth, they may reflect arousal states associated with pitch movements (e.g., see Scherer, 2003). Large intervals typically reflect 
dramatic moments in music: if a performing musician experiences this dramatic effect as heightened arousal, then facial expressions associated with that affective state might naturally arise. Such facial expressions may not always be evident, however; elite singers, for example, may actively suppress facial expressions that negatively impact on the quality of their vocal output.

Thompson, Russo, and Livingstone (2010) probed the nature of these visual cues in more detail. Participants were asked to judge the size of a sung interval from a video recording of a musician singing various ascending melodic intervals. By overlaying a mask onto the video, parts of the face were occluded in some conditions. They determined that participants were able to accurately judge interval size even when only head movements were visible (all facial features occluded). The ability to evaluate interval size was increased further when facial features were visible, suggesting that subtle movements of individual facial features provide detailed information about melodic structure. Three-dimensional motion capture was next used to track the movements of the singers. Movement analyses confirmed that the magnitude of head displacement and mouth opening correlated well with actual interval size (the size as sung by the performer), corroborating the results of Thompson and Russo (2007).

What processes underlie such effects? Do viewers consciously consider head and facial movements when judging interval size or is there an unconscious process by which auditory and visual input are automatically integrated? To answer this question, participants were asked to perform a distracting secondary task (counting digits that appeared on the screen) while judging sung interval size from audio-visual recordings of singers (Thompson et al., 2010, Experiment 2 and 3). The secondary task was designed to occupy attentional resources thereby reducing the capacity of participants to use attentional resources to judge interval size. The presence of the secondary task did not alter the effect of visual information. That is, judgements of interval size were modulated according to the type of visual information present (congruent or incongruent), and this effect was observed regardless of the difficulty of the secondary task, or whether participants were explicitly 
told to ignore visual signals. These results suggest that visual information is automatically and pre-attentively combined with auditory information, and that our experiences and interpretations of music represent a balance between these cues.

\subsection{The influence of gesture on affective judgements}

The movements produced by musicians also carry emotional information. Thompson et al. (2005) examined whether the perceived emotional valence of a sung melodic interval is influenced by the facial expressions adopted by the singer. Audio-visual recordings of a sung minor third (usually associated with a negative emotional valence) and a sung major third (usually associated with a positive emotional valence) were edited to yield congruent audio-visual conditions (e.g., sung minor interval combined with facial expressions used to produce that same minor interval) and incongruent conditions (e.g., sung minor interval combined with facial expressions used to produce a major interval). By comparing judgements for congruent and incongruent conditions, it was possible to determine that facial expressions significantly influenced the perception of emotional valence in sung intervals. That is, a performing musician can significantly alter the emotional valence of music merely with accompanying facial expressions.

This effect was present even when participants were engaged in a distracting secondary task and when they were explicitly asked to ignore facial expressions and make their judgements exclusively on the auditory information (Thompson, Russo, \& Quinto, 2008). As with the influence of facial expressions on the perceived size of a melodic interval, the influence of facial expressions on the perceived valence of music occurs pre-attentively, without the need to consciously attend to facial cues. This influence is not restricted to isolated sung intervals but is also evident for longer and more naturalistic performances. Thompson et al. (2005, Experiment 5) examined the influence of facial expressions and gestures on emotional judgements of excerpts from sung performances by Judy Garland. Groups of participants were shown either an audio-visual excerpt or an audio-only excerpt from each performance. Participants then judged the emotional valence of the perfor- 
mance using a Likert scale (sad to happy). The group exposed to the audio-visual recording was instructed to base their judgements exclusively on the audio signal alone. Emotional judgements differed significantly depending on whether listeners had been exposed to the complete audio-visual excerpt or only the audio component. That is, the emotional quality of the performance was again influenced by the presence of visual information (even though the group exposed to audio-visual materials was instructed to ignore the visual information). The direction of this effect was dependent on the excerpt presented, suggesting that Judy Garland used facial expressions differently depending on her emotional goals for the song. For some songs, the inclusion of facial expressions and gestures led to an increase in the perceived emotional valence (judged more happy); in other excerpts it led to a decrease in perceived emotional valence (judged more sad).

The preceding studies indicate that the facial expressions and gestures of performers can profoundly affect the way music is perceived, interpreted, and experienced. Movements and facial expressions can shape the perception of musical structure (interval size) as well as the emotional messages communicated by the music. What are the mechanisms by which such movements exert their influence? One hint comes from the observation that music listening is rarely, if ever, a passive activity. Indeed, recent evidence suggests that movements produced by the perceiver - a kind of "mirroring" behaviour - might play a part in how the music is perceived, and may in turn influence the emotional character.

Livingstone, Thompson, and Russo (2009) employed an emotional-production synchronisation paradigm whereby participants were asked to observe and then imitate sung phrases. The phrases were sung with varying emotional intentions (along with a neutral control condition), and participants were asked to pay close attention to the emotional content of the sung phrases as they observed them so that they could then imitate the expressed emotion. The facial expressions of the observers were monitored during the observation phase, immediately after the observation phase as they planned their own imitation (pre-production phase), during their imitation of the sung phrase (production phase), and after their imitation (post-production phase). Facial expressions were 
monitored with three-dimensional motion capture (Experiment 1 ) and electromyography (EMG; Experiment 2).

For the motion capture analysis, markers were placed on the eyebrows, lip corners, and lower lip. Movements of the eyebrows and lip corners reflect eyebrow furrowing and smiling, respectively. Movements of the lower lip should occur during any vocalisation, but these movements do not tend to vary with emotional intentions. Analyses revealed that there was an effect of emotion on the movement of the eyebrow marker during the planning, production, and post-production phases. This suggests that movement related to the production of "sad" facial expressions begin before participants start to imitate sung phrases with "sad" emotional connotations. With the lip corner marker, the effect of emotion was present in all four phases, including the perception phase. This suggests that movements related to the production of "happy" facial expressions begin not only prior to production but even while participants are merely observing sung phrases.

Motion capture analysis was supplemented by the use of EMG to measure activity of zygomatic muscles (near the cheeks) and corrugator muscles (near the eyebrows), which are associated with positive and negative affect, respectively (Dimberg, Thunberg, \& Elmehed, 2000; Schwartz, Fair, Salt, Mandel, \& Klerman, 1976). As part of the analysis, a composite score was calculated by summing together zygomatic muscle activity while observing or imitating a happy sung phrase with corrugator muscle activity while observing or imitating a sad sung phrase. This analysis indicated that emotional facial expressions occurred during perception, pre-production, production, and post-production phases. As with the motion capture data, the EMG data suggest that observing emotional singing can immediately trigger a process of emotional mimicry. However, as participants in this investigation were explicitly instructed to imitate sung phrases, additional research is needed to determine whether emotional mimicry for sung phrases occurs automatically. 


\subsection{Music and action}

The experimental findings outlined above can be explained within a theoretical framework that grounds perception within action. Psychologists have long been interested in the link between perception and action. For example, William James (1905) argued strongly that perception and action were tightly intertwined. According to the ideomotor principle outlined by James (1905), actions are represented in terms of their perceptual consequences. Such action-perception associations operate in two directions: an action plan can trigger a representation of the perceptual outcome of that action; and perceiving an action can trigger action plans that lead to such a perceptual outcome. Gibson (1986) also viewed perception and action as mutually dependent on one another. According to this "ecological" approach, the environment is perceived in terms of the action possibilities available to the organism, called affordances. For example, a cup might be perceived in terms of the grip that would be required to manipulate it, and a chair might be perceived in terms of the act of sitting down. Furthermore, Gibson (1986) observed that actions themselves are often used to extract valuable information from the environment. Along similar lines, the common-coding hypothesis argues that there is a common representational code for perception and action (e.g., see Hommel, Müsseler, Aschersleben, \& Prinz, 2001). Perception leads to the formation of action plans, and action plans lead to perceptual predictions. Several lines of evidence have been used to support this hypothesis.

Experiments on stimulus response compatibility provide some of the most well known examples of perception influencing action planning (e.g., Simon, 1969). Stimulus response compatibility refers to the way in which the perception of certain stimuli can activate action plans. Drost, Rieger, Brass, Gunter, and Prinz (2005) measured reaction times for pianists who played a musical chord in response to a visual stimulus. The visual stimulus depicted the chord that was to be played, but it was also accompanied by one of the following auditory stimuli: (a) the same chord (congruent); (b) a different chord (incongruent); or (c) non-tonal sounds (control). The results showed that pianists responded faster to the visual stimulus when it was accompanied by a congruent auditory 
signal. That is, perceiving the predicted sensory consequence of an action (the sound of the chord) was sufficient to activate action plans for the production of the corresponding action, thereby priming and expediting the execution of the action.

The connection between perception and action has also been examined directly by measuring the effects of perception on motor regions of the brain. This approach has revealed action plans in the brain in the absence of any physical movement. Grahn and Brett (2007) found that listening to musical rhythms resulted in activation of motor regions in the brain, including the supplementary motor area, dorsal premotor regions, and the basal ganglia. Importantly, there was no activation of primary motor regions suggesting that motor activation was not related to actual movement, but action plans. They were also able to exclude motor preparation as a possible cause of motor activation by focusing their analysis on experimental trials in which no motor planning or preparation was required. Not only do rhythmic stimuli activate motor regions in the brain, neurological diseases that destroy motor regions in the brain can lead to deficits in rhythm discrimination. Grahn and Brett (2009) found that patients with Parkinson's disease perform poorly on a rhythmic discrimination task when compared with healthy controls. Thus, not only does a regular beat induce movement and activate the motor system, a functional motor system might be required for veridical perception of a regular beat.

Overlapping patterns of activity in motor regions have also been observed for perception and performance tasks. Bangert et al. (2006) have shown that motor regions are activated when musicians either listen to music or perform actions related to music making. In this study, musicians and non-musicians either listened to novel music or arbitrarily pressed keys on a silent piano keyboard. Musicians showed significantly more motor activity than non-musicians during the listening tasks. Similarly, Haueisen and Knösche (2001) found that when pianists listened to piano sequences, there was activation in motor regions normally associated with the production of piano sequences. These results suggest that a common motor network underlies the perception and the performance of music. 
Recent neurophysiological evidence has illustrated the ubiquity of common coding for perception and action. For example, specialised neurons, called canonical neurons, have been shown to be active both when a monkey performs a particular action, such as a precision grip, and when they observe an object that can be manipulated by means of a precision grip (Fadiga, Fogassi, Gallese, \& Rizzolatti, 2000). That is, the mere observation of an object directly generates an action plan for interacting with that object.

Another class of perceptual-motor neurons called mirror neurons are selective, not for objects, but for actions. Mirror neurons are active both during the observation of actions performed by conspecifics and during the production of action (for a review, see Rizzolatti \& Craighero, 2004). That is, merely perceiving an action is sufficient for generating action plans in the observer. The activity of mirror neurons has also been observed following the perception of action effects, including the sounds that are produced by actions (Kohler et al., 2002). These auditory mirror neurons (or echoic neurons) may play an important role in music perception. Indeed, regions thought to contain mirror neurons were shown to be active during the music perception tasks studied by Grahn and Brett (2007) and Bangert et al. (2006), outlined above. Mirror neurons do more than simply copy the actions that are perceived: they also help to predict the consequences of observed actions (Wilson \& Knoblich, 2005).

Common-coding theory states that perception of action influences action planning, and action (and action planning), in turn, influences perception. Repp and Knoblich (2007) found that trained pianists were more likely to report two ambiguous tones as ascending in pitch if they produced those tones with a left-to-right key press sequence on a piano keyboard. That is, participants were more likely to hear an ascending pitch sequence when they pressed the piano keys in an order that was compatible with an ascending pitch sequence. Similarly, Phillips-Silver and Trainor (2007) found that movements made concurrently with musical stimuli influenced how those stimuli were perceived, encoded, and subsequently remembered. They trained participants to make rhythmic (bouncing) body movements in time with rhythmically ambiguous sounds. The body movements could be 
produced in either duple or triple form. After the training session, they asked participants to identify the sounds that they heard during the training session. Even though all participants were presented with rhythmically ambiguous sounds during the training sessions, participants were more likely to select sounds that explicitly conformed to the rhythmic pattern of the movements they made during the training session. That is, the rhythmic patterns of participants' movements shaped their perceptions of the acoustic signal.

If the perception of action can generate action plans, and action plans can modulate perception, then it stands to reason that the perception of action can modulate perception. That is, our action systems may modulate the way in which we perceive actions, or auditory stimuli accompanied by actions. The most commonly cited example is the McGurk effect (McGurk \& Macdonald, 1976) outlined above (Section 6.2). However, the empirical studies performed by Thompson and colleagues, detailed earlier (Section 6.2 and 6.3), also provide evidence for this claim. In these examples, the observation of facial expression and gestures activate action representations that modulate the incoming acoustic signal such that sung melodic intervals accompanied by sad facial expressions (facial expression produced when singing a minor third) are sadder sounding than the same sung intervals accompanied by "happy" facial expressions (facial expression produced when singing a major third). Considered within the common-coding framework, the findings of Thompson and colleagues can be explained by grounding emotional experience in the body. Observing the body movements and facial expressions of a musical performer should lead to the activation of action plans in the observer. These action plans should, in turn, modulate the perception of the expressive content contained in the audio-visual signal. Furthermore, the findings of Livingstone et al. (2009), which suggest that observers initiate action plans while merely observing emotional singing, also supports the notion that bodily actions help listeners "get the feel" of the music. 


\subsection{Action and emotion}

Action-based accounts of emotion have a long history. The theory of emotion developed by James (1884) directly linked emotions with sensory-motor processes in the brain. James (1884) noted that emotions are tightly correlated with particular patterns of bodily change. For James (1884), it is not our emotional states that lead to bodily changes such as a racing heart. Rather, "bodily changes follow directly the perception of the exciting fact, and ... our feeling of the same changes as they occur is the emotion" (p. 449). This assumption led James (1884) to further argue that, "[w]e feel sorry because we cry, angry because we strike, afraid because we tremble" (p. 450). James also asked his readers to imagine a strong emotion, and then try and remove from this imagining all bodily changes. Without those bodily changes, he believed that there would be nothing left of the emotion.

Other authors have also emphasised the link between action and emotion. For example, Frijda (1987) equated each emotion with a particular profile of action tendencies, called an action readiness profile. The emotion of happiness might be associated with an increased "tendency to stay in proximity of" and "exuberance [or] free activation, [and] increased generalised action readiness" (p. 133). The perceptual theory of emotion developed by Jesse Prinz (2005) combined the views expressed by James (1884) and Frijda (1987). On this account, the link between emotion and the body (and action) is bi-directional. That is, emotions are perceptual states registering bodily change but they also motivate bodily changes and control action. Behavioural and neuroimaging studies have supported the notion that emotional experience is grounded in action and the body. Zuckerman, Klorman, Larrance, and Spiegel (1981) found that participants reported increased intensity of emotional experience when asked to produce exaggerated facial expressions while observing emotionally laden video clips. Similarly, Strack, Martin, and Stepper (1988) found that participants' ratings for the "funniness" of cartoons was attenuated by restricting facial movements by asking participants to either hold a pen between their lips or the teeth. Holding a pen in the lips prevented the participants using their zygomatic muscles (used for smiling) because this would have resulted in the pen falling from the mouth. Thus, 
it is possible to infer the emotional state one is in by registering the bodily changes that one experiences. And these bodily changes can modulate, or interfere, with the emotional content of perceived emotions in, for example, the music.

Support for grounding the phenomenology of emotion in the body also comes from patients with spinal cord lesions. In a study of 25 patients with various types of spinal injuries, Hohmann (1966) found that sustaining an injury that decreased sensation from the body led to changes in the phenomenal character of emotions. Nearly all patients reported a decrease in the emotional feelings of fear and anger after their injury, and it was found that, where these emotions remained, they were primarily abstract and detached in nature and lacking the somatic phenomenal descriptions that had usually been associated with them.

Neuroimaging has also implicated somatosensory regions in emotional experience. In a study by Damasio et al. (2000), participants were asked to recall an emotional episode from their life while undergoing brain scanning using positron emission tomography. Although different patterns of neural activity were observed for the different emotions that were recalled, all emotions activated regions associated with body perception, such as the somatosensory cortex, and regions receiving sensory signals from the viscera.

Links between music and action are increasingly incorporated into theories of the emotional power of music. The Shared Affective Motion Experience (SAME) model of musical emotion proposed by Molnar-Szakacs and Overy (Molnar-Szakacs \& Overy, 2006; Overy \& Molnar-Szakacs, 2009) draws heavily on the common coding of action-perception and action-production performed by mirror neurons. They argue that the mirror-neuron system is a key mechanism that allows emotion to be transmitted by music. The mirrorneuron system is thought to provide access to a musician's emotional state by representing music in terms of motor commands. Auditory signals that arise from music are imbued with emotional intentions because they imply actions that are themselves emotional. When auditory signals are transformed into a motor representation in a listener's brain, the listener can "work backwards" from this motor representation to the emotional 
intentions that gave rise to the music.

In this view, music is perceived as an "intentional, hierarchically organised sequence of expressive motor acts" (Overy \& Molnar-Szakacs, 2009, p. 492). How is this done? Most listeners lack the specialised knowledge needed to infer the specific motor acts required to produce musical sounds. For example, only a highly trained pianist can appreciate the fine-grained movements required to perform a Bach fugue on the piano. Overy and Molnar-Szakacs (2009) proposed that depending on the listener's level of musical ability, they can extract movement information at different levels of the motor system. Whereas a skilled musician may have access to fine-grained actions used to perform music, a musically unskilled listener may interpret emotional intentions with respect to gross actions such as clapping or tapping, which mirror the overall beat. In support of this model, Overy and Molnar-Szakacs (2009) cited the tight links between music and movement, along with evidence demonstrating that music can directly activate motor regions in the brain.

An important assumption of the SAME model is that emotional intentions are inferred directly from this motor activity. This assumption is derived from the early "mind reading" model of mirror neuron function proposed by Gallese and Goldman (1998). Although the idea that we have neurons specialised for mind-reading is intriguing, it has been heavily criticised. In particular, Jacob and Jeannerod (2005; see also Jacob, 2008) have argued that the perception-action transformations performed by mirror neurons cannot underlie the process of mind-reading (or inferring intentions from actions) because the motor command replication performed by mirror neurons is not sufficient for mental state replication. Intentions and actions have a many-to-one relation, so it is rarely possible to "work backwards" from an action to an intention. A wide range of intentions can lead to the same motor response. For example, the act of picking up a pen may occur because an agent is sad and is writing a note of sympathy about a deceased friend, or because an agent is angry and has decided to tender a resignation. Similarly, identical scalpel movements can be used by a doctor trying to save a patient's life or by a madman torturing a victim 
(Jacob \& Jeannerod, 2005).

Moreover, mirror neuron activity is not necessary to understand the intentions behind actions. In particular, experiments have shown that observers readily provide mentalistic explanations for the behaviour of agents in displays consisting of simple geometric shapes (e.g., see Castelli, Happè, Frith, \& Frith, 2000). Simple geometric shapes lack human bodies and, therefore, do not lend themselves to mirror neuron activity. Furthermore, electrophysiological evidence (e.g., Umiltà et al., 2001) suggests that, rather than decoding the goal of an action, mirror neuron activity is dependent on the observer already understanding the goal of an observed action.

While this evidence suggests that mirror neurons are neither necessary nor sufficient for mind-reading, it is still possible that mirror neurons may form part of a larger system for inferring intentions from actions. However, this hypothesis awaits further evidence. We believe there is a more parsimonious way to account for the interconnections between music, the mirror-neuron system, and emotion. Specifically, we argue that the action plans generated by mirror neurons function for perceptual prediction.

Consistent with this argument, Wilson and Knoblich (2005) propose that mirror neurons form part of an emulator system used for predicting the actions of other agents. They suggest that during action observation, mirror neurons are used to simulate the actions of others by mapping the actions of observed agents onto an internal model of the observer's own motor system. This internal model can then be used to stand in for actual sensory input and this allows an observer to track the movements of an observed agent in real-time-even when they are obscured from view-or to generate predictions of the unfolding motor act. Thus, mirror neuron activity may be used to predict the sensory consequences of observed motor action (Flach, Knoblich, \& Prinz, 2003; Knoblich, Seigerschmidt, Flach, \& Christensen, 2002) or it may be used to plan anticipatory actions in response to unfolding observed motor acts. This system may underlie the ability to engage in synchronous action (e.g., Chapter 2) including ensemble music performance (Keller, Knoblich, \& Repp, 2007). 
Action production need not be overt. It can also take the form of simulated perception-action couplings. The ability of music (as human action) to engage processes of synchronisation may represent one of the defining characteristics of music experience and may be critical for generating emotional responses to music.

\subsection{Expectancy, action, and emotion}

Having examined the role of action in how we perceive the emotional quality of music, we now turn our attention to how the same system can lead to the arousal of emotions. The power of common coding of perception and action, and synchronisation driven by motor simulation, is that it provides a framework for linking action-based accounts of music perception with action-based and prediction-based (expectation) accounts of musical emotion. If perception is linked to action plans and action plans are also linked to perceptual prediction, then action-based and expectation-based accounts of musical emotion are two sides of the same coin.

The landmark work of Meyer (1956) draws heavily on melodic expectancies as the psychological basis for emotional responses to music. For Meyer (1956), musical meaning was conveyed by the manner in which one musical event built expectancies for musical events to follow. Similarly, Huron's (2006) ITPRA theory outlines multiple ways that expectancy (prediction) can account for emotional responses to music. Huron (2006) proposed five subsystems of musical expectancies that are relevant to the emotional power of music: imagination, tension, prediction, reaction, and appraisal. The first two subsystems mediate responses that occur before the expected/unexpected event (pre-outcome responses) and are related to guiding behaviour by means of predicted future emotional experiences and physiological and psychological preparation for predicted future events. The final three subsystems mediate responses that occur after the event (post-outcome responses), and their activity is related to whether the event was accurately predicted, as well as pre-attentive and conscious assessment of the event once it has occurred.

The pre-outcome response of imagination is an important motivator for action. It can 
be used to preview future events and to predict/simulate what these future events might feel like and what emotional responses they may evoke. In this way, imagination is used to generate and test out action plans and simulate the emotional consequences of these action plans. These simulated emotional states act as a reward or punishment that can guide action selection. The pre-outcome response of tension refers to preparation for an expected event. Preparation may involve preparing cognitive resources through heightened attention or it may take the form of increasing action readiness, through physiological arousal, in preparation for predicted future events.

After the occurrence of the event the prediction response provides a reward for accurate prediction or a punishment if there was a failure to predict the event. As suggested by the common-coding hypothesis, the action plans generated by the musical stimulus, and pre-outcome responses, may drive these predictions. A positively-valenced emotional response serves as a reward and a negatively-valenced emotional response serves as a punishment. The emotional experience evoked by the prediction response serves to fine-tune the predictive mechanisms so that future predictions are more accurate. The prediction response is not dependent on the nature of the event; it merely depends on whether the event was accurately predicted.

The nature of the event (whether it was a positive or negative event) drives two postoutcome responses-reaction and appraisal. Reaction responses are rapid assessments of the events that occurs pre-attentively and outside of conscious awareness. Reaction responses may also take the form of mild surprise evoked by violations of expectancies, and can result in action consequences such as rapid "knee-jerk" bodily actions or visceral responses. Appraisal responses are deliberate, conscious assessments of an event that operate over longer time frames and are heavily dependent on context. They operate independently of reaction responses, and reaction responses that may initially evoke a negatively-valenced emotional response may, upon reflection or appraisal, turn into a positively-valenced emotional experience. For example, initial negative feelings evoked by violations of harmonic expectancies may, upon reflection, be appreciated on intellec- 
tual grounds. Because of the independent action of these different response subsystems, Huron (2006) argued that expectancies and prediction are able to generate complex, dynamic, and highly nuanced emotional experiences.

Perceptual predictions are directly and bi-directionally linked with action plans. Therefore, expectancies such as those described in Huron's (2006) model may be functionally equivalent to action plans. Indeed, Huron (2006) explicitly links action with preoutcome responses, such as tension, and post-outcome responses, such as reaction. As such, expectancy-based accounts of musical emotion may be seen as a special case of more general action-based accounts of emotion.

\subsection{A feedback-controlled synchronisation model}

Recently, work by Carver and Scheier (2009) has sought to develop a general account of the link between action and emotion. Key to Carver and Scheier's (2009) framework is the notion that behaviour is goal-directed and controlled by feedback processes. Emotional experiences are the natural outcomes of the attainment or the failure to attain goals. The feedback generated by the attainment or failure to attain goals is fed into a behaviour control system in order to modify future behaviour. Goals may include static end-states (e.g., catching a ball or passing an exam) or dynamic goals that evolve over time. Goal-directed behaviour may also take the form of generating expectancies, where the goal of an expectation is the accurate prediction of the environment. Synchronising covert or overt bodily actions with music may be a particularly important form of goal-directed behaviour that occurs during music listening. Synchronisation relies on expectancies that are derived from predictive action plans generated by the musical signal. These expectancies need to be constantly updated so as to match the ongoing dynamic music signal. By unifying expectancies and goal-directed action, Carver and Scheier's (2009) model can account for many of the same phenomena discussed in Huron's (2006) model of expectancy in music.

In any system, whether artificially engineered or biological, the maintenance of goaldirected behaviour occurs through feedback control. Feedback control systems can be bro- 
ken down into four parts: an input value, a reference value, a comparison mechanism, and an output. Inputs provide a measure of the current state of the system or a prediction or expectancy that the system has generated. The reference value serves as a target value with which the input is compared. This reference value, or goal state, can take the form of an action goal or it can be a measure of the value that the system is attempting to predict. A comparison process then computes the discrepancy between the input value and the reference value and generates an error signal. The error signal is then sent as output to be fed back into the controller of the system. The output signal can then be used to modify the behaviour of the system or to update the predictions that the system is generating.

The behaviour of the system can be modified in one of two ways. In positive feedback systems, the output signal modifies the system's behaviour so as to increase the discrepancy between the input value and reference value. This type of feedback system is used to guide the behaviour of a system away from a possibly negative stimulus. In negative feedback systems, the output modifies the behaviour of the system so that the input values approach the reference value. In the case of predictive systems, which generate expectancies, negative feedback is used, because expectancies have the goal of accurate prediction or minimal discrepancy between the predicted values/events and actual values/events.

In Carver and Scheier's (2009) model, positive and negative feedback do not directly generate emotional experience. Instead, the valence of an emotional experience is determined by the action of a secondary loop that runs simultaneously and in parallel with the primary, or behaviour-guiding, loop. This secondary, or affect, loop monitors the progress of the behaviour-guiding loop, with the error signal generated by the primary loop serving as the input for the affect loop. In the case of negative feedback systems, the affect loop monitors the rate at which the primary loop's error signal is decreasing over time, corresponding to the progress that the system is making towards achieving its goal. Carver and Scheier (2009) suggest that the error signal that is generated by this second loop manifests phenomenologically as an experience of emotion. Valence is determined by comparing the pace or rate of change in the error signal from the primary loop to a 
criterion or expected rate of change. If the discrepancy is being reduced at a rate that is better than expected, then the error signal generated by the affect loop leads to a positive emotional experience. If the discrepancy is decreasing at a rate that is worse than expected, then the error signal generated by the affect loop leads to a negative emotional experience. Thus, positive and negative emotional experiences indicate to the system how well the behaviour-guiding loop is doing at achieving its goal.

Carver and Scheier's (2009) model provides a plausible mechanism underlying emotional effects arising from music expectancies (see also, Thompson \& Quinto, in press). Perceptual experiences that occur during music listening activate action plans and, hence, expectancies. More generally, music engages processes of synchronisation that are underpinned by dynamic action-prediction-action-production loops. This is evident in cases where people feel compelled to move "in time" with music.

Within the common-coding framework, listening to music is functionally equivalent to engaging in goal-directed behaviour. By manipulating how goals are achieved and violated, composers control valence and arousal dimensions of emotional experience. The activity of the primary (behaviour-guiding) loop, which is directly activated by action plans initiated by music listening, may allow composers to control the arousal dimension of emotional experience. The activity of the secondary (affect) loop means that the emotional valence of music shifts as listeners or performers are able to increase or decrease their level of synchronisation over time. Together, the actions of the behaviour-guiding and affect loops create a rich and complex emotional experience.

Figure 6.1 provides a sketch of the synchronisation feedback model. The model places the performing musician front and centre in the emotional experience. By observing or imagining the actions of performers, audiences generate action plans and expectancies through the common-coding of perception and action. Action plans and expectancies work together to drive the process of synchronisation. The level at which listeners can synchronise is dependent on their level of motor experience. Skilled musicians may be capable of detailed and fine-grained synchronisation and can generate detailed expectan- 


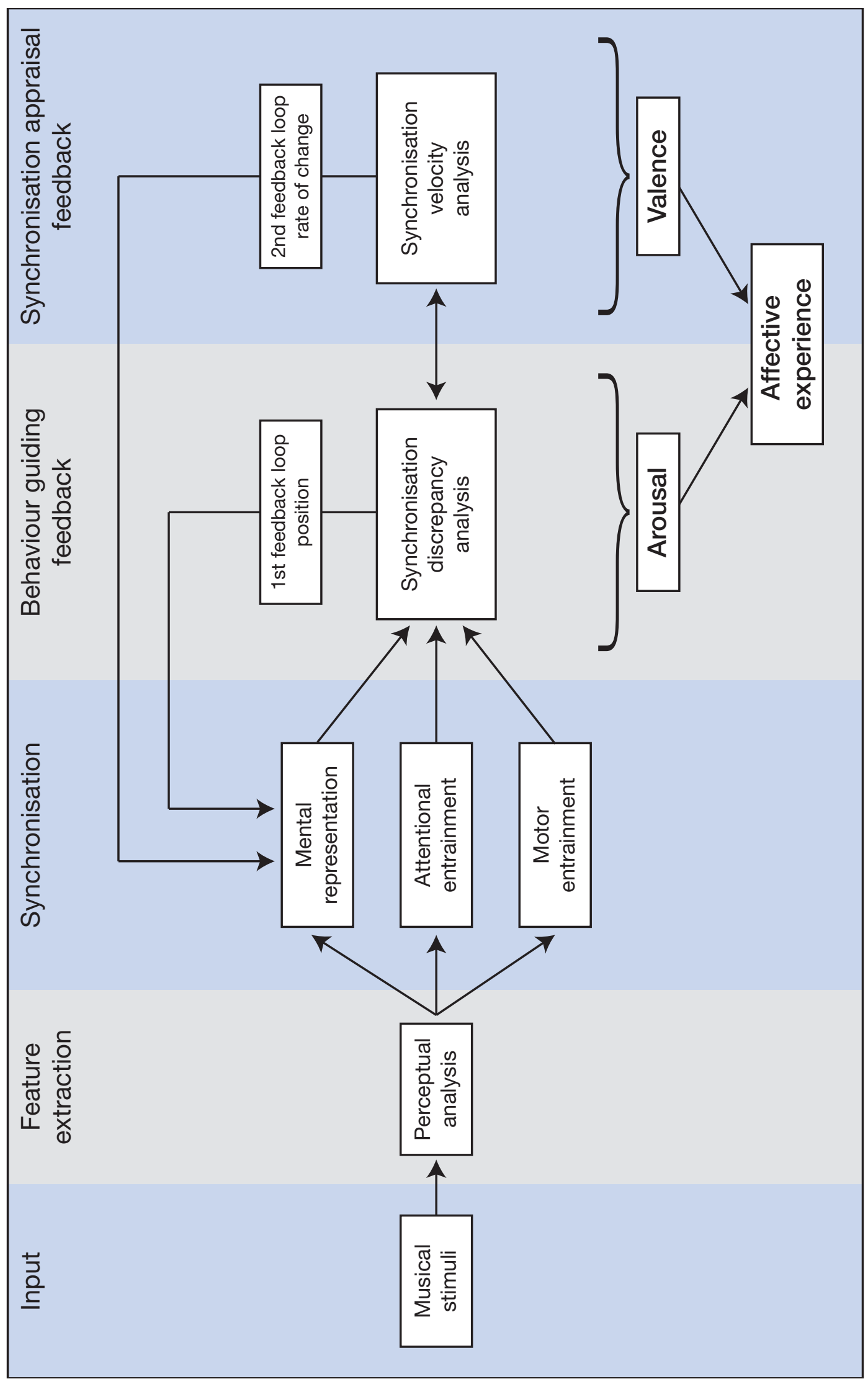


cies. Unskilled, musically naïve listeners may instead synchronise using gross actions such as clapping, tapping, and dancing or by synchronising with expressive movements by performing actual or simulated movements, such as "air guitar" (see also, Schubotz, 2007). Musical expectancies also arise from observing or inferring the actions of performers. Surprising or predictable actions in the stream of patterned motor activity that make up a musical signal are just as effective at evoking emotions as surprising or predictable acoustic events. By controlling the interplay between the fulfilment and violation of these expectancies along with the fulfilment and violation of expectancies generated by the acoustic signal (as in the case of B. B. King or Judy Garland, detailed in Section 6.2 and 6.3), performers can evoke a dynamic and highly nuanced emotional experience.

Musical expectancies need not only occur for immediate effects. Some expectancies can occur over larger time scales and can possess more abstract content. For example, immediate expectancies may occur as a direct result of action plans generated by the beat, or tactus, of a musical piece. Simultaneously, expectancies related to higher order melodic, harmonic, or phrasing structure may also be generated. The results of more immediate expectancies may interact with these higher order expectancies in order to create a multi-layered emotional experience. Furthermore, bodily changes induced by music's effect on the motor system may operate on a longer time scale in order to modulate the phenomenology of emotional experiences evoked by processes of synchronisation. Thus, the emotions aroused by the music are not necessarily the same as those that we perceive the music as expressing. The model of expression we endorse states that we infer emotions from the motor activations that result from the actions we take to be responsible for producing the music. Meanwhile, our model of emotion states that one's emotional valence is due to one's success or failure at predicting the musical action. It is possible then for one to have a positive emotional experience in response to predicting musical action that is perceived as emotionally negative. It is the confluence of these effects that may account for the powerful emotional responses to music. 


\subsection{References}

Bangert, M., Peschel, T., Schlaug, G., Rotte, M., Drescher, D., Hinrichs, H., ... Altenmüller, E. (2006). Shared networks for auditory and motor processing in professional pianists: evidence from fMRI conjunction. NeuroImage, 30(3), 917-926.

Carver, C. S., \& Scheier, M. F. (2009). Action, affect, and two-mode models of functioning. In E. Morsella, J. A. Bargh \& P. M. Gollwitzer (Eds.), Oxford handbook of human action (pp. 298-327). Oxford: Oxford University Press.

Castelli, F., Happè, F., Frith, U., \& Frith, C. (2000). Movement and mind: a functional imaging study of perception and interpretation of complex intentional movement patterns. NeuroImage, 12(3), 314-325.

Damasio, A. R., Grabowski, T. J., Bechara, A., Damasio, H., Ponto, L. L. B., Parvizi, J., \& Hichwa, R. D. (2000). Subcortical and cortical brain activity during the feeling of self-generated emotions. Nature Neuroscience, 3(10), 1049-1056.

Dimberg, U., Thunberg, M., \& Elmehed, K. (2000). Unconscious facial reactions to emotional facial expressions. Psychological Science, 11(1), 86-89.

Drost, U. C., Rieger, M., Brass, M., Gunter, T. C., \& Prinz, W. (2005). Action-effect coupling in pianists. Psychological Research, 69(4), 233-241.

Fadiga, L., Fogassi, L., Gallese, V., \& Rizzolatti, G. (2000). Visuomotor neurons: ambiguity of the discharge or 'motor' perception? International Journal of Psychophysiology, 35, 165-177.

Flach, R., Knoblich, G., \& Prinz, W. (2003). Off-line authorship effects in action perception. Brain and Cognition, 53(3), 503-513.

Freeman, W. (2000). A neurobiological role of music in social bonding. In N. L. Wallin, B. Merker \& S. Brown (Eds.), The origins of music (pp. 411-424). Cambridge MA: MIT Press.

Frijda, N. H. (1987). Emotion, cognitive structure, and action tendency. Cognition \& Emotion, 1(2), 115-143. 
6. Music, action, and affect

Gallese, V., \& Goldman, A. (1998). Mirror neurons and the simulation theory of mindreading. Trends in Cognitive Sciences, 2(12), 493-501.

Gibson, J. J. (1986). The ecological approach to visual perception. New York: Psychology Press.

Grahn, J. A., \& Brett, M. (2007). Rhythm and beat perception in motor areas of the brain. Journal of Cognitive Neuroscience, 19(5), 893-906.

Grahn, J. A., \& Brett, M. (2009). Impairment of beat-based rhythm discrimination in Parkinson's disease. Cortex, 45(1), 54-61.

Haueisen, J., \& Knösche, T. R. (2001). Involuntary motor activity in pianists evoked by music perception. Journal of Cognitive Neuroscience, 13(6), 786-792.

Hohmann, G. W. (1966). Some effects of spinal cord lesions on experienced emotional feelings. Psychophysiology, 3(2), 143-156.

Hommel, B., Müsseler, J., Aschersleben, G., \& Prinz, W. (2001). The theory of event coding: a framework for perception and action planning. Behavioral and Brain Sciences, 24(5), 849-878.

Huron, D. (2006). Sweet anticipation: music and the psychology of expectation. Cambridge MA: MIT Press.

Jacob, P. (2008). What do mirror neurons contribute to human social cognition? Mind \& Language, 23(2), 190-223.

Jacob, P., \& Jeannerod, M. (2005). The motor theory of social cognition: a critique. Trends in Cognitive Sciences, 9(1), 21-25.

James, W. (1884). What is an emotion? Mind, 9(34), 188-205.

James, W. (1905). The principles of psychology. New York: Henry Holt and Company.

Keller, P. E., Knoblich, G., \& Repp, B. H. (2007). Pianists duet better when they play with themselves: on the possible role of action simulation in synchronization. Consciousness and Cognition, 16(1), 102-111.

Knoblich, G., Seigerschmidt, E., Flach, R., \& Christensen, W. (2002). Authorship effects in the prediction of handwriting strokes: evidence for action simulation during action 
perception. The Quarterly Journal of Experimental Psychology: Section A, 55(3), 10271046.

Kohler, E., Keysers, C., Umiltà, M. A., Fogassi, L., Gallese, V., \& Rizzolatti, G. (2002). Hearing sounds, understanding actions: action representation in mirror neurons. Science, 297(5582), 846-848.

Livingstone, S. R., Thompson, W. F., \& Russo, F. (2009). Facial expressions and emotional singing: a study of perception and production with motion capture and electromyography. Music Perception, 26(5), 475-488.

McGurk, H., \& Macdonald, J. (1976). Hearing lips and seeing voices. Nature, 264(5588), 746-748.

Meyer, L. B. (1956). Emotion and meaning in music. Chicago: University of Chicago Press.

Molnar-Szakacs, I., \& Overy, K. (2006). Music and mirror neurons: from motion to 'e'motion. Social, Cognitive, and Affect Neuroscience, 1(3), 235-241.

Overy, K., \& Molnar-Szakacs, I. (2009). Being together in time: musical experience and the mirror neuron system. Music Perception, 26(5), 489-504.

Phillips-Silver, J., \& Trainor, L. J. (2007). Hearing what the body feels: auditory encoding of rhythmic movement. Cognition, 105(3), 533-546.

Prinz, J. J. (2005). Passionate thoughts: the emotional embodiment of moral concepts. In D. Pecher \& R. A. Zwaan (Eds.), Grounding cognition: the role of perception and action in memory, language, and thinking. New York: Cambridge University Press.

Repp, B. H., \& Knoblich, G. (2007). Action can affect auditory perception. Psychological Science, 18(1), 6-7.

Rizzolatti, G., \& Craighero, L. (2004). The mirror-neuron system. Annual Review of Neuroscience, 27(1), 169-192.

Scherer, K. R. (2003). Vocal communication of emotion: a review of research paradigms. Speech Communication, 40(1-2), 227-256.

Schubotz, R. I. (2007). Prediction of external events with our motor system: towards a new framework. Trends in Cognitive Sciences, 11(5), 211-218. 
Schwartz, G., Fair, P., Salt, P., Mandel, M., \& Klerman, G. (1976). Facial muscle patterning to affective imagery in depressed and nondepressed subjects. Science, 192(4238), $489-491$.

Simon, J. R. (1969). Reactions toward the source of stimulation. Journal of Experimental Psychology, 81(1), 174-176.

Strack, F., Martin, L. L., \& Stepper, S. (1988). Inhibiting and facilitating conditions of the human smile: a nonobtrusive test of the facial feedback hypothesis. Journal of Personality and Social Psychology, 54(5), 768-777.

Thompson, W. F. (2009). Music, thought, and feeling : understanding the psychology of music. Oxford: Oxford University Press.

Thompson, W. F., \& Quinto, L. (in press). Music and emotion: psychological considerations. In E. Schellekens \& P. Goldie (Eds.), Philosophy and aesthetic psychology. Oxford: Oxford University Press.

Thompson, W. F., \& Russo, F. A. (2007). Facing the music. Psychological Science, 18(9), 756757.

Thompson, W. F., Graham, P., \& Russo, F. A. (2005). Seeing music performance: visual influences on perception and experience. Semiotica, 156, 203-227.

Thompson, W. F., Russo, F., \& Quinto, L. (2008). Audio-visual integration of emotional cues in song. Cognition \& Emotion, 22(8), 1457-1470.

Thompson, W. F., Russo, F. A., \& Livingstone, S. R. (2010). Facial expressions of singers influence perceived pitch relations. Psychonomic Bulletin \& Review.

Umiltà, M. A., Kohler, E., Gallese, V., Fogassi, L., Fadiga, L., Keysers, C., \& Rizzolatti, G. (2001). I know what you are doing: a neurophysiological study. Neuron, 31(1), 155165.

Wilson, M., \& Knoblich, G. (2005). The case for motor involvement in perceiving conspecifics. Psychological Bulletin, 131(3), 460-473. 
Zuckerman, M., Klorman, R., Larrance, D., \& Spiegel, N. (1981). Facial, autonomic, and subjective components of emotion: the facial feedback hypothesis versus the externalizer-internalizer distinction. Journal of Personality, 41(5), 929-944. 



\section{Part V.}

Conclusions 

CHAPTER 7

\section{Conclusions}

This thesis has argued against the notion that perception and action are merely distinct non-cognitive input and output systems. Instead, it has argued for the view that perception and action are intimately connected and inseparable from (at least certain aspects of) cognition. In particular, it has argued for a primary role for action emulation in the cognitive mechanisms that underlie the control of interpersonal interaction. While other theorists have also argued that action emulation plays a role in social cognition, the position defended here is importantly different. For example, Rizzolatti, Fogassi, and Gallese (2001) identify a key role for action emulation in social cognition when they argue that action emulation is involved in action understanding. They defend the position that "we understand [the actions of others] when we map the visual representation of the observed action onto our motor representation of the same action" (Rizzolatti et al., 2001, p. 661; see also Chapter 1 , Section 1.4.1). In contrast to the view that action emulation is involved in action understanding, I have shown that action emulation is critical for our ability to predict the actions of other agents (Part II) and that this prediction ability plays a central role in our ability to engage in interpersonal coordination (Part III). Furthermore, I have 


\section{Conclusions}

shown that action emulation, and its predictive function, might also play an important role in music cognition and might help to explain how music is able to create powerful emotional experiences (Part IV). In the section that follows, each of these are discussed in turn.

\subsection{Part II: An empirical investigation of action prediction}

Part II investigated the role played by action emulation in our ability to predict the actions of others in a series of experiments using a visual-motor action synchronisation paradigm. In particular, Part II investigated three outstanding issues within the empirical literature. Chapter 2 examined whether the action emulation system comes on-line rapidly enough for it to plausibly be the basis of interpersonal action prediction during joint action. Chapter 3 examined the nature of the internal model that is used during action prediction by examining the effect of internal and external action information on prediction performance. Meanwhile, Chapter 4 ruled out an alternative explanation-self-similarity in action production tempo-that has been suggested for the results of Chapter 2, and similar studies (e.g., Flach, Knoblich, \& Prinz, 2003). In the section that follows, the contribution made by each of the chapters to our understanding of action prediction will be discussed in turn.

\subsubsection{Chapter 2: Action synchronisation with biological motion}

If action emulation is to be a plausible mechanism by which co-actors can coordinate their actions, then the action emulation system must come on-line rapidly. If the emulator system takes a long time to come on-line, then this raises doubts about its role in joint action coordination because this would suggest that there must be a second system that can be used for joint action coordination in the absence of action emulation. To investigate the functioning of the emulator system, Chapter 2 employed a self/other synchronisation task. The action emulation hypothesis states that observers use their own action produc- 
tion systems to generate predictions about observed action. Therefore, a prediction of this hypothesis is that predictions should be more accurate when there is a better match between the action system used for initially producing the action and the action system used in the subsequent prediction task. When generating predictions about recordings of self-produced actions there is (in principle) a perfect match between the system used to initially produce the actions and the system used for the subsequent prediction tasks. The self-synchronisation advantage, or authorship effect, is taken as an index of the emulator system. Importantly, in Chapter 2, subjects viewed stimuli of moving bodies and not merely the sensory effects produced by human action (e.g., Flach et al., 2003). It was predicted that this manipulation would result in the action emulation system coming on-line more rapidly.

The results confirmed that action prediction was indeed more accurate when subjects generated predictions about recordings of their own actions. Importantly, and unlike previous experiments that have used a similar paradigm (Flach et al., 2003), the results presented in Chapter 2 did not reveal a delay in the emergence of the authorship effect. Rather, the authorship effect was present from the first experimental block. Thus, the results of Chapter 2 provide further support for the notion that action prediction is achieved by means of motor emulation. Importantly, as the experiment presented in Chapter 2 was able to overcome the delay in the emergence of the authorship effect observed by Flach et al. (2003), these results also provide the first demonstration that action emulation mechanisms can come on-line rapidly thus suggesting that these mechanisms could plausibly underlie joint action coordination.

\subsubsection{Chapter 3: Motor experience modulates limb and joint information}

Chapter 3 investigated the nature of the action model used during action prediction by manipulating subjects' access to an internal representation of the action and by manipulating the nature of the external representation of the action available to the subject. Action prediction might be achieved by means of two different types of internal mod- 


\section{Conclusions}

els: first, a general purpose mechanism that, while implemented in the motor system, does not require the observed actions to be mapped onto the observer's body; and second, by mapping the observed action onto an internal model for that (specific) action. To examine the nature of the model, two manipulations were designed to facilitate the process of mapping the observed action onto the observer's body. Motor experience was manipulated to provide some observers with an internal representation of the action onto which they could map the stimulus. Stimulus information content was also manipulated by constructing two sets of stimuli. The first set of stimuli contained all the movement information required to perform the synchronisation task, but contained no information about the action used to produce the movement. The second set of stimuli contained an actual depiction of the action used to produce the movement.

The results showed that including information about the limbs and joints in the stimuli-that is, using stimuli that can be directly mapped onto the observer's body in a partby-part manner-results in an increase in prediction accuracy. However, this effect was modulated by motor experience, as it was only found for the group of subjects that had motor experience performing the task. This suggests that observers need an internal representation of the action onto which to map the observed action. These results are consistent with the idea that experienced observers are able to map the observed action onto their internal representation for that action, while naïve observers may instead only simulate the general dynamics of the stimulus. Since naïve observers do not map the observed action onto an internal representation of that action, including information in the stimulus to enhance the accuracy of the mapping process has no effect. In addition, the results also showed that experienced observers are better able to respond to fine-grained local variations in stimulus timing, while naïve observers tend to respond to the global dynamics of the stimulus. This is also consistent with naïve observers only simulating the general dynamics of the stimulus and experienced observers simulating a more complete description of the action that is better able to capture these fine-grained local timing variations. 


\subsubsection{Chapter 4: The effect of tempo and dynamic cues}

Chapter 4 investigated an alternative explanation for the results presented in Chapter 2 (see also Flach et al., 2003) that performance on an action synchronisation task is not due to the operation of an emulator system, but is primarily determined by a similarity in the preferred tempo for movement production in the initial movement phase and the subsequent synchronisation phase. In these previous experiments, subjects have been asked to synchronise responses with particular time points in a recording of a rhythmic action. These recordings could either be of self-produced or other-produced actions, and the results indicated that subjects show superior synchronisation performance for self-produced action. This effect is ordinarily interpreted as support for the hypothesis that observers use a forward model of their own action dynamics to generate predictions about the observed action. However, there is an alternative explanation for these results. Studies on spontaneously produced rhythmic actions (for a review, see Fraisse, 1982) have shown that individuals have a preferred rhythm for producing rhythmic actions and, therefore, it might be possible to explain the results of self/other synchronisation experiments in terms of a match between the preferred tempo for action production during the initial movement phase and the subsequent synchronisation phase.

Chapter 4 (Experiment 1 ) ruled out this alternative explanation by comparing synchronisation accuracy for stimuli that had the same tempo but differed in terms of the stimulus dynamics (velocity profile) between the synchronisation points. The results showed that synchronisation accuracy is not equal for tempo-matched stimuli. Rather, synchronisation accuracy was enhanced for stimuli that conform to a natural human velocity profile between the synchronisation points. This supports the notion that action prediction is more accurate when the dynamics of the stimulus more closely match the dynamics of human movement. This is consistent with the theory that action prediction relies on action emulation.

It might also be possible to provide an alternative explanation for the results of Experiment 1 that does not invoke action emulation. The superior prediction accuracy for 


\section{Conclusions}

the natural velocity stimuli might merely have been a result of these stimuli containing a deceleration phase before the synchronisation points, which was missing in the constant velocity stimuli. Two subsequent experiments (Experiment $2 \mathrm{a}$ and $2 \mathrm{~b}$ ) were able to rule out this explanation by showing that the deceleration phase does not provide an unambiguous cue for action synchronisation. To demonstrate this, two additional pairs of constant velocity and natural velocity stimuli were created. In one set of natural velocity stimuli there was a low degree of inter-stimulus variability (Experiment 2a) while in the other set of natural velocity stimuli there was a high degree of inter-stimulus variability (Experiment 2b). The results showed that deceleration cues can enhance synchronisation accuracy, but only when there is little variability in between the stimuli. That is, deceleration cues were only effective when subjects were given sufficient opportunity to learn, and thus internally replicate, the dynamics of the stimulus. These results are consistent with the notion that action synchronisation is dependent on a mechanism that involves mapping the observed stimulus onto an internal action representation that is capable of replicating the dynamics of the stimulus. When variability is low, the mapping process is easier because any initial inaccuracies in the mapping process can be corrected by subsequent exposure to stimuli with similar dynamics. However, when variability is high, this process is more difficult because the initial attempts to map the stimuli onto an action representation may be inaccurate. When an adequate internal representation of the action cannot be created, deceleration and other dynamic cues do not provide any performance advantage on the synchronisation task.

\subsection{Part III: A theoretical framework for joint action}

\subsubsection{Chapter 5: From action control to joint action}

Part II argued that action emulation underlies the ability to predict the actions of other actions. Chapter 5 extended this argument and demonstrated that the same mechanisms that underlie individual action control might also be adept at explaining socially coor- 
dinated action. The framework presented in Chapter 5 argued that socially coordinated action is mediated by an emulator system, implemented within the action control system, which allows actors to emulate the action control systems of their co-actors and, thus, coordinate joint actions in much the same way as individual actions. This was demonstrated in three ways: First, by showing that actors represent the subgoals of their co-actors in much the same way as they represent their own goals-that is, joint subgoal representations influence action planning and action execution in much the same way as individual goal representation influences individual action planning and action execution. Second, by showing that predicting the actions of others relies on the same mechanisms that are employed to predict one's own actions during action control. And third, by showing that being able to predict the actions of others and integrate those predictions into action planning allows groups of individuals to perform tasks in the same manner as individuals would perform the same task when acting alone.

The final section of Chapter 5 extended the framework further and sketched out an account of the phenomenology of joint action. Predictive mechanisms in individual action control have been used to explain the sense of agency over individual action (Carruthers, 2010; Frith, Blakemore, \& Wolpert, 2000). For example, the comparator model of agency suggests that the sense of agency over actions can be explained using forward models for action. According to the comparator account, when the forward models correctly predict produced actions a sense of agency is felt over these actions. As these predictive mechanisms might also underlie joint action coordination, this raises the possibility that these mechanisms might also feature in an account of the phenomenology of joint action. In particular, these mechanisms might mediate feelings of group flow (Sawyer, 2006), where actors report a feeling of being able to predict the actions of co-actors. Furthermore, these mechanism might also underlie more dramatic phenomena, such as feelings of "oneness", "boundary loss", and feelings of agency over the collective action, that are reported by musicians engaged in ensemble music performance (e.g., see Berliner, 1994; McNeill, 1995; Monson, 1996). While this framework still needs to generate experimentally tractable 


\section{Conclusions}

predictions, it might provide a useful starting point for trying to understand these phenomena.

\subsection{Part IV: Action synchronisation in other contexts}

\subsubsection{Chapter 6: Music, action, and affect}

Part IV provided an important extension to the work presented in Part II and III, by demonstrating that action emulation might also be used to explain other social phenomena, namely musically-evoked emotional experiences. Chapter 6 outlined a model that aims to explain how music is able to create powerful emotional experiences in the listener. Rather than considering music as a purely auditory phenomenon, Chapter 6 argued that music is more accurately construed as a multi-modal experience that includes auditory, visual, kinaesthetic, and motor dimensions. Furthermore, it is an embodied experience (see Chapter 1, Section 1.1.2, for more on embodied cognition), and motor processes, in particular predictive motor processes such as those outlined in Part II and III, play a central role in music cognition and the ability of music to create emotional experiences. Chapter 6 also argued that music listening should not be thought of as asocial. Indeed, for much of history, before the advent of recording technology, music was an intensely social phenomenon often involving synchronised clapping, drumming, chanting, and dancing (Freeman, 2000), and with no clear distinction between audience and performer.

Chapter 6 demonstrated the multi-modal, embodied nature of music by reviewing evidence that merely observing the actions produced by musicians can influence the perception and interpretation of the acoustic dimension of music. Following this, and as shown in Part II, Chapter 6 argued that observation of action is capable of engaging predictive sensory-motor processes in observers and listeners. Emotional experience has been grounded in action and, therefore, Chapter 6 argued that it might be possible to ground emotional the nature of music in the motor processes that are engaged during music listening. That is, it might be possible for listeners to infer emotions from the mo- 
tor activation that results from generating predictions about the music. In the case of music, prediction and expectancy has also been linked to emotional experience, and the framework presented in Chapter 6, therefore, allows for a unification of expectancy-based models of musical emotion and action-based models of emotion.

\subsection{Implications and directions for future research}

The work presented in this thesis provides further support for the notion that the way in which we perceive the world and, in particular, the way in which we perceive other people, is fundamentally shaped by our own action capabilities and the body we possess.

The results also have important implications for our study of music cognition. In particular, they suggest that much can be gained by understanding how music engages sensorymotor systems. That is, rather than focusing purely on the acoustic signal, the study of music cognition demands an embodied perspective that takes seriously the role played by the body and its action capabilities in shaping musical experience.

The results of this thesis also demonstrate the need to move beyond purely analytic approaches to studying joint action (e.g., Bratman, 1993; Searle, 1990). While these approaches may prove useful in understanding certain types of joint action they tend to focus on the product of joint action while neglecting the process. Purely analytic approaches may also fail to capture the essence of joint actions such as ensemble music and dance because they neglect the way in which these joint activities are constructed in real-time through the tight coupling of the perception and action systems of multiple individuals.

While the account of action prediction presented here is particularly suited for understanding joint-action coordination in dyads, it remains to be seen how well this approach will generalise to situations where more than two people are interacting. In particular, future research should examine whether people are capable of emulating the actions of more than one individual in parallel. It is possible that people do not emulate the actions of multiple agents in parallel but instead merely emulate the collective "other" within a single model using more general purpose predictive mechanisms. An extension of the 


\section{Conclusions}

prediction paradigm presented here, where observers generate predictions about two or people acting concurrently, may be capable of addressing this question.

Additionally, the account of joint agency presented in Chapter 5 will also need to be experimentally verified. The account predicts feelings of joint agency when actors are able to accurately predict the actions and action-effects of their co-actors, and while the reports of musicians support this account, properly controlled experimental work is still needed. Joint effect production tasks may be particularly suited to this goal.

Csibra (2008) and Wilson and Knoblich (2005) have suggested that action prediction relies crucially on the mirror-neuron system, but there is still no direct evidence that the effects reported here (e.g., the authorship effect) rely on the activity of the mirror-neuron system. Future research will need to make explicit the link between the mirror-neuron system and action prediction. Some preliminary work has already been conducted towards making this link. In particular, in an extension of the paradigm presented here, an electroencephalographic study by Colling, Thompson, Sutton, and Williams (2011) required subjects to view visually identical displays of animated mannequins under two conditions. In the prediction condition, subjects were asked to synchronise a button-press with the movement of a mannequin (see Part II), while in the control condition subjects were asked to synchronise a button-press with a fixation cross that intermittently changed colour. The results showed increased activity in the mirror-neuron system, as indexed by mu-rhythm suppression (see Hari, 2006), during the mannequin prediction condition. This suggests that predicting the actions of conspecifics preferentially engages the mirrorneuron system. Future research, however, will need to examine whether differences in mirror-neuron system activity also correlate with differences in prediction accuracy. The authorship effect might prove a useful tool for investigating this question.

This thesis aimed to show that understanding the links between perception and action are indispensable if we are to develop a full account of social phenomena. What is needed is more than just a study of the social mind; we must not neglect the social body. It is hoped that this thesis has made one modest step towards this goal. 


\subsection{References}

Berliner, P. F. (1994). Thinking in jazz: the infinite art of improvisation. Chicago: University of Chicago Press.

Bratman, M. E. (1993). Shared intention. Ethics, 104(1), 97-113.

Carruthers, G. (2010). A problem for wegner and colleagues' model of the sense of agency. Phenomenology and the Cognitive Sciences.

Colling, L. J., Thompson, W. F., Sutton, J., \& Williams, M. A. (2011, July). Functional equivalence between acting alone and acting together. Paper presented at the 4th Joint Action Meeting, Vienna, Austria.

Csibra, G. (2008). Action mirroring and action understanding: an alternative account. In P. Haggard, Y. Rossetti \& M. Kawato (Eds.), Sensorimotor foundations of higher cognition (pp. 435-459). New York: Oxford University Press.

Flach, R., Knoblich, G., \& Prinz, W. (2003). Off-line authorship effects in action perception. Brain and Cognition, 53(3), 503-513.

Fraisse, P. (1982). Rhythm and tempo. In D. Deutsch (Ed.), The psychology of music (pp. 149-180). New York: Academic Press.

Freeman, W. (2000). A neurobiological role of music in social bonding. In N. L. Wallin, B. Merker \& S. Brown (Eds.), The origins of music (pp. 411-424). Cambridge MA: MIT Press.

Frith, C. D., Blakemore, S. J., \& Wolpert, D. M. (2000). Abnormalities in the awareness and control of action. Philosophical Transactions of the Royal Society B: Biological Sciences, $355,1771-1788$.

Hari, R. (2006). Action-perception connection and the cortical mu rhythm. In C. Neuper \& W. Klimesch (Eds.), Event-Related dynamics of brain oscillations (pp. 253-260). Elsevier.

McNeill, W. (1995). Keeping together in time : dance and drill in human history. Cambridge MA: Harvard University Press. 
7. Conclusions

Monson, I. (1996). Saying something : jazz improvisation and interaction. Chicago: University of Chicago Press.

Rizzolatti, G., Fogassi, L., \& Gallese, V. (2001). Neurophysiological mechanisms underlying the understanding and imitation of action. Nature Reviews: Neuroscience, 2(9), $661-670$.

Searle, J. (1990). Collective intentions and actions. In P. R. Cohen, J. Morgan \& M. E. Pollack (Eds.), Intentions in communication (pp. 401-415). Cambridge MA: MIT Press.

Wilson, M., \& Knoblich, G. (2005). The case for motor involvement in perceiving conspecifics. Psychological Bulletin, 131(3), 460-473. 


\section{Part VI.}

Appendix 

APPENDIX A

Cognitive science does not reduce to neuroscience

\section{A.1. Introduction}

Classical cognitive scientists have argued that the goal of cognitive science is to uncover facts about mental information-processing systems responsible for producing intelligent behaviour. For this reason, facts about the brain are seen as irrelevant because they have no bearing on the nature of information processing. To use the words of one prominent advocate of this position, "[n]o amount of knowledge about the hardware of a computer will tell you anything serious about the nature of the software that the computer runs. In the same way, no facts about the activity of the brain could be used to confirm or refute some information-processing model of cognition" (Coltheart, 2004, p. 22). Neuroscientists too have sought to understand intelligent behaviour, with some in the field adhering to an extreme philosophical position that largely "refuse[s] the psychological any genuine causal explanatory role" (Bickle, 2003, p. 115), reduces mind to brain, and affords higher level theories and methods only a "heuristic role... in the search for lower mech- 


\section{A. Cognitive science does not reduce to neuroscience}

anisms and reductions" (Bickle, 2003, p. 130). ${ }^{1}$ These two approaches-cognitive science and neuroscience- - use different methods and vocabularies and, while some have argued that they are pitched at different levels of explanation and have distinct explanatory targets (e.g., Coltheart, 2004), others have argued for a single explanatory target and have suggested that psychology should be reduced to neuroscience (e.g., Bickle, 2003).

The aim of this paper is to examine these two viewpoints from the perspective of the cognitive psychologist. In the first half, we will examine the role that neuroscience and neuroscientific reasoning can play in theorising about cognition. We will suggest that studying the brain can and does tell us important facts about information-processing systems, and that neuroscience can generate novel behavioural predictions that can be incorporated into cognitive theories. In the second section, we aim to defend cognitive psychology from reduction to neuroscience by examining how intelligent behaviour relies crucially on extra-neural structures and the environment in that the organism is embedded.

\section{A.2. The role of neuroscience}

As seen above, classical cognitive science has often been dismissive of neuroscience. This view stems, in part, from a philosophical commitment to the principle of multiple realisability. We will first examine the claim of multiple realisability to see how it is used to protect psychology from reduction. Following this, we will examine some of the problems with multiple realisability. In doing so, we aim to show that once a commitment to multiple realisability is abandoned the clear division between structure and function begins to fade.

\footnotetext{
${ }^{1}$ Bickle claims that the reductionism he favours is the reductionism found in actual neuroscientific practice. This is a controversial claim. However, it will be Bickle's reductionism that is the primary focus of the present paper.
} 


\section{A.2.1. Multiple realisability}

Multiple realisability has been one of the most prominent defences against reduction of the special sciences (any science above physics). Those who have favoured reductionism have either had to explicitly deal with multiple realisability within their reductionist program or have had to question its truth (e.g., Bickle, 2003). However, a commitment to multiple realisability within a non-reductionist program might do more than simply ward off reductionism; it might also create an artificially strong divide between implementation and function thereby obscuring important functional consequences that arise directly out of particular details of physical implementation.

Multiple realisability forms a central part of the functionalist tradition of philosophy of mind. Functionalism is the idea that mental states (or rather, cognitive processes) should be defined by the functional role that they play rather than how they are physically realised. For example, the cognitive process of reading aloud might be defined as the cognitive process that has the function of transforming printed letter strings into vocalisations of the words that correspond to those printed letter strings. Multiple realisability, simply stated, is the idea that one function, such as word reading, can be realised, or implemented, by many different, physically distinct, systems. If two functionally isomorphic systems (maintaining a particular set of input-output relations) can be physically distinct, then nothing useful can be learnt about the function itself by merely studying the physical makeup; doing so will only give details about how the function is implemented.

To understand the problem with multiple realisability, it is necessary, following Shapiro (2000), to introduce the concepts of grain of analysis and of causally relevant properties. These terms can be made clear by way of an example. Two mousetraps, functionally isomorphic, serve the function of catching mice. That is, they take mice as their input, and by a particular set of internal state transitions, produce caught mice as their output. One mousetrap has a spring-loaded bar made from copper while the other has a spring-loaded bar made from steel. Here then are two physically distinct systems that both perform the function of catching mice. Do they count as two distinct realisations of a single functional 


\section{A. Cognitive science does not reduce to neuroscience}

kind? We would argue that, in any meaningful sense, they do not. The metal, the copper or the steel, is not a causally relevant property of a mousetrap. That is, it is a physical property that, when changed, does not alter how the mousetrap performs its function. What is causally relevant about the bar is that it is rigid. To be sure, steel and copper are distinct realisations of the property of rigidity, but, as Shapiro (2000) suggests, these differences are not relevant to their function in the mousetrap. We can screen off these differences and they need not enter our explanation of the mousetrap. When a causally relevant property is changed it brings about a change in how the mousetrap performs its function. For example, one mousetrap could use a spring-loaded bar that snaps shut to trap and kill the mouse. If we were to physically alter this mechanism and replace it with a box with a trap door that closes thereby trapping the live mouse instead, we have altered a causally relevant property of the trapping mechanism as we have altered how the mousetrap performs its function. However, again there is a question of whether the two mousetraps should count as distinct physical realisations of a single functional kind. On one grain of analysis the answer would be yes. Both mousetraps realise the function of catching mice. On a finer grain of analysis, however, the answer is not so clear. One mousetrap catches and kills mice, while the other catches mice alive. These two mousetraps could be realisations of two distinct functional kinds-a live mouse catcher and a mouse killer. Thus, for two mousetraps (or brains or computers) to be distinct realisations of a single functional kind, they would need to differ in terms of their causally relevant parts while remaining functionally isomorphic at the grain of analysis of interest.

\section{A.2.1.1. Fine-grained analysis}

The grain of analysis problem is clearly evident when contrasting two different approaches to artificial intelligence: good old fashioned artificial intelligence (GOFAI) with nonGOFAI approaches, including connectionism. The GOFAI approach to artificial intelligence has its roots in the work of Newell and Simon (e.g., Newell \& Simon, 1997) and, in particular, the law of qualitative structure for symbol systems. This law states, "a physical 
symbol system has the necessary and sufficient means for general intelligence" (p. 87). A symbol system is a type of computer that might be realised as a desktop computer or a biological brain. What matters for intelligent behaviour is not whether the system is made from silicon-stuff or brain-stuff, but rather that it is a physical symbol system. But what is a physical symbol system? Simply put, a physical symbol system is a physical system consisting of symbols (tokens) that can be joined together to form symbol structures such that these symbol structures can be manipulated through processes such as, for example, concatenation, separation, creation, and duplication, according to the various rules (or syntax) of the system. Thus, a simple binary adder might be considered a form of a physical symbol system because it takes symbols (binary ones and zeros) and by joining them together using a simple logical rule (for example, logical AND) it produces the "intelligent" behaviour of adding. Similarly, the human brain might also be a physical symbol system because it contains symbols (brain states) that form symbolic structures manipulated according to syntactic rules. Therefore, it is claimed that what matters for cognitive science is that human brains are physical symbol systems, and whether the symbols are particular physico-chemical brain states or binary digits realised by electromagnetic switches is not relevant to our understanding of intelligent behaviour.

To be sure, there have been many successes within the GOFAI paradigm, and it certainly seems that at a coarse grain of analysis some aspects of human intelligence may be approximated by a physical symbol system. But is this also the case at a finer grain?

Approaches to artificial intelligence, such as connectionism, differ from GOFAI in a number of key respects. Primary among these is a lack of emphasis on symbolic manipulation. Instead, connectionist modellers place a greater emphasis on network structure, and they may refer to the nodes in their network being neuron-like or their network models being neurally plausible. However, to say that connectionism emphasises structure over function would be a misrepresentation of the true value of connectionism. As mentioned earlier in our discussion of mousetraps, causally relevant physical properties can have functional consequences, and this is certainly the case when comparing biological 


\section{A. Cognitive science does not reduce to neuroscience}

brains and connectionist networks on the one hand with GOFAI approaches on the other. These functional differences are most clear at a finer grain of analysis.

A complete treatment of connectionism is outside of the scope of the current paper; however, for our current purposes it will be sufficient to highlight a few areas in which a connectionist structure has microfunctional consequences- that is, how implementation constrains function in cognitive systems at a fine grain of analysis.

Human brains are biological systems, and human minds are the products of biological systems. Viewing the mind through the lens of the biological brain puts greater emphasis on certain functional features of minds that might otherwise be ignored by an abiological cognitive science. It has been said that "[n]othing in biology makes sense except in the light of evolution" (Dobzhansky, 1973, p. 125). Why should it be that biological cognition is different? We claim that it is not. There are a number of constraints that restrict the nature of possible computational solutions that can be employed to problems routinely faced by biological organisms. These constraints may result in microfunctional (or finegrained functional) differences between biological (or biologically inspired) cognition and non-biological cognition. Furthermore, the way evolutionary processes work also means that the final solution to some complex computational problem is constrained by the more basic capacities upon which it is built. That is, microfunctional properties of human cognition may arise from the fact that complex capacities are built out of simpler solutions to more evolutionarily basic problems. Other constraints faced by biological cognitive systems might include how the system behaves when damaged, how the system copes under conditions of degraded input, or the ability to employ its cognitive resources under a wide range of circumstances (Clark, 1989). These constraints are brought into sharp focus by considering cognition as a biological phenomenon.

Primary among the constraints faced by biological cognition is time. That is, processing must be able to take place on a time scale that is appropriate for a particular biological organism in order for that organism to survive in its environment. Thagard (1986) argues that there is a selective pressure in favour of fast computation and that if our minds had 
operated substantially slower then "our species would never have evolved" (p. 305). Thus, the temporal properties of a cognitive system should enter into our functional description. Approaches to artificial intelligence based on parallel computation recognise this constraint while those that favour serial computation (such as GOFAI approaches) do not (Thagard, 1986). Now, if it is the case that only machines built using parallel architectures (that is, architectures that are brain-like) are capable of meeting the time constraints imposed on biological cognition then it is the case that structure can be a causally relevant factor in determining microfunctional properties. Thagard (1986) claims that this is the case and that these temporal properties arise as a direct result of the network structure favoured by connectionism.

Another microfunctional property to arise from connectionist networks is crosstalk. Crosstalk refers to the errors that arise due to partial activation of units corresponding to similar representations. For example, in the CID model of word perception (McClelland, 1985), presenting two words simultaneously to the network results in the activation patterns from both words being combined. This can cause interference between the activation patterns of the two words and can cause partial activation of units corresponding to other words that share letters with the two original words. This interference causes a characteristic pattern of errors. So, for example, if the network is shown the words SAND on the left and LANE on the right and then "asked" to report the word on the left the network may respond with SANE. This migration error, where the E from LANE migrates to SAND, arises directly out of the structure of the CID model. Originally, McClelland thought that this might be a drawback of the CID model; however, far from being a drawback of the model, it is actually a positive aspect because this pattern of errors mimics those produced by humans (e.g., Mozer, 1983). The important point here is that this microfunctional property arises directly out of the architecture of the network. It is important, however, not to overstress the match between the architecture of CID networks and real brains, but this example serves to show that there is not a clear, sharp division between function and implementation. Rather, these lines are blurred and they will continue to 


\section{A. Cognitive science does not reduce to neuroscience}

blur as more is learnt about neuroscience and as more is learnt about the microfunctional features of cognition.

Science does not happen in a theoretical vacuum. The theoretical baggage that the cognitive scientist carries into an experiment not only affects how they might interpret data, but it also affects which observations might count as usable data and which might be discarded as noise or as irrelevant (Lakatos, 1970). Studying how it is that brains implement the functions they do serves to refocus the cognitive scientist's attention on aspects of the data that might otherwise be neglected or ignored. For example, time constraints on biological cognition might be ignored out of a philosophical commitment to multiple realisability and observations of particular error patterns might be regarded as noise rather than valuable sources of data. What the preceding examples aim to show is that facts about the brain can yield scientific progress in developing models of cognition and can produce novel behavioural predictions. Models that cannot account for speed of computation, computation under natural environmental conditions, and various other microfunctional properties of natural human cognition, are not satisfactory models of human cognition. In our discussion of connectionism, it is not our intention to give the impression that connectionism is the final word on cognitive modelling. To be sure, connectionism is only one set of tools that can be employed in order to understand better the functional consequences that result from particular structural implementations. More detailed, and more biologically realistic, models of neural computation-possibly incorporating cellular and molecular details - are needed and it would be premature to set an a priori limit below which structural details may be screened off as functionally irrelevant for explaining intelligent behaviour. Neuroscience, and a biological view of cognition, here serves the purpose of highlighting those physical details (e.g., network-like structure) that might be important for understanding functional properties of cognitive processes and microfunctional features of intelligent behaviour. 


\section{A.3. The role of brain imaging}

Not only can "brain-like" computation produce novel behavioural predictions, so too can techniques that measure actual brain functioning in behaving humans. The rise of brain imaging has been met with much scepticism within certain segments of the cognitive science community. Much of this criticism is for good reason, but to draw conclusions about brain imaging's in principle inability to answer questions about the mind from its methodological failings would be too hasty. Coltheart (2004) identifies two goals of neuroimaging: localisation of cognitive functions and using neuroimaging data "to make theoretical decisions entirely at the cognitive level" (p. 21). As an example, included in this second goal Coltheart lists using neuroimaging to judge between different cognitive models of some cognitive system — a goal which Coltheart believes imaging has (so far) utterly failed in achieving. However, there is another possible use for neuroimaging: we suggest that the real power of neuroimaging lies in using neuroscientific data for generating and developing cognitive models that, stripped of neuroscientific language, can be pitched solely at the functional level ${ }^{2}$.

The discovery of mirror neurons is an example of both the poor use of neuroscientific data and the valuable role neuroscientific data can play in generating theories about cognition. Mirror neurons, which were originally discovered in monkey premotor cortex, have been implicated in many cognitive tasks ranging from facilitating observational learning to understanding the goals and intentions of others (for a brief review, see Rizzolatti, 2005). Many of these proposals have been very speculative and have gone well beyond what the data suggest; however, not all proposals have fallen into this trap. The real value of the discovery of mirror neurons has been that is has stimulated new ways, or at least reinvigorated old ways, of thinking about cognition, by emphasising the intimate links between perception, action, and cognition. Action-centred cognition has a long history going as far back, at least, as James (1905), and action was also at the core of the eco-

\footnotetext{
${ }^{2}$ Although we have stripped the neuroscientific language from the example outlined below, it is still up for debate whether neuroscientific language should be stripped from cognitive explanations - we choose to avoid this debate in the present paper.
} 


\section{A. Cognitive science does not reduce to neuroscience}

logical psychology of Gibson (1986). What mirror neurons have added to the debate is that they provide direct evidence of the links between perception, action, and cognition; this marks a clear shift from the amodal, action-neutral, symbolic approach to cognition found in classical cognitive science.

An example of how mirror neuron theory can be turned into a (purely) cognitive theory can be found in the emulator theory of action prediction put forward by Wilson and Knoblich (2005). Wilson and Knoblich (2005) suggest that the mirror-neuron system provides a means by which an observer can predict the actions of an observed agent by internally simulating, or emulating, the agent's actions. That is, they propose that a system (consisting of mirror neurons) exists that has the purpose of generating real-time simulations of the movement dynamics of observed agents. These simulations can stand in for information from the external world, thereby bypassing the delay that would be introduced by waiting for actual sensory input, and these predictions can then be used to drive behaviour.

In developing the emulator theory of action prediction, Wilson and Knoblich (2005) point to the properties of mirror neurons that provide the basis for their ideas. For example, they cite evidence from fMRI (e.g., see Buccino et al., 2001) that shows that action observation in humans results in somatatopic activation of premotor areas that are also active during action execution (this is consistent with single unit recording studies in monkeys). Stated functionally, this is evidence that action execution and action perception share a common neural code. Furthermore, Wilson and Knoblich (2005) point to evidence from PET (Stevens, Fonlupt, Shiffrar, \& Decety, 2000) suggesting motor and parietal regions (known to contain mirror neurons) are preferentially active for biologically possible movements over biologically impossible movements. In addition, Wilson and Knoblich (2005) also point to evidence from fMRI that shows mirror neuron activity when observing skilled movements is contingent on the observer being similarly skilled (e.g., see Calvo-Merino, Glaser, Grézes, Passingham, \& Haggard, 2005). Stated in cognitive psychological terms, this suggests that observers represent the actions of observed 
agents by mapping the agent's actions onto their own motor repertoire. Last, they point to evidence from single unit recording studies in monkeys (the nature of these experiments make them difficult to replicate in humans) that show mirror neuron activity to be predictive in nature, with neurons that fire during observation of grasping firing even when the final movement is obscured from view, suggesting that dynamic action-centred representations of other agents exhibit a type of representational momentum. That is, they extrapolate action beyond what is actually perceived. Taken together, these functional descriptions can be used to generate a purely functional/cognitive theory that generates behavioural predictions (which might not otherwise have been thought of) that can be tested with behavioural data. For example, it can be predicted that if observers predict the actions of others by mapping observed actions onto their own action repertoires, then it may be possible to detect the effects of the observer's action repertoire on the predictions they generate. In particular, action predictions should be more accurate when observed actions are more easily mapped onto the observer's action repertoires. That is, predictions should be more accurate when there is a better match between the observer's and the observed agent's action systems. This prediction has been tested in several experiments using a self/other prediction paradigm. (The logic behind this paradigm is that the selfprediction condition provides a perfect match between the observer's and the observed agent's action repertoire while the other-prediction condition does not). Evidence from behavioural studies confirms this prediction of the emulator theory (e.g., see Chapter 2; Flach, Knoblich, \& Prinz, 2003).

As more details about properties of mirror neurons come to light, these can be incorporated into the (neuro) cognitive emulator model and further novel behavioural predictions can be generated. For science to progress it is not enough to only falsify old theories with new empirical observations and to develop new theories that can account for the excess empirical content. Development of new theories that lead to the prediction of novel, or unexpected, facts (that are later corroborated) is also vitally important (Lakatos, 1970). Thus for neuroimaging to inform our theorising about mind and behaviour it need not 
(only) be used to falsify cognitive theories, but it can also lead to scientific progress when it predicts novel behavioural effects.

\section{A.3.1. A non-reductive role for neuroscience}

In the preceding section, we highlighted two examples of how data from neuroscience can be used to inform cognitive theorising. First, we showed that in certain cases structural properties of the brain can have functional consequences for cognition. Viewing cognition through the lens of biology can help to highlight those functional properties that might be missed by an abiological cognitive science. Second, we demonstrated that novel behavioural predictions can be generated based on findings from the neuroimaging literature, suggesting that the demands which Coltheart imposes on neuroimaging-that of distinguishing between two competing cognitive theories - fail to appreciate neuroimaging's value to the discipline of cognitive psychology. By viewing neuroimaging in this light, it places constraints on the possible information processing models for a particular cognitive function proposed by cognitive psychologists and serves to highlight those aspects of behavioural data that may be important. This, however, does not amount to a reduction to neuroscience as it allows for a conservation of the terminology, language and, most importantly, the methods of cognitive science. Some have argued that it is not enough to explain intelligent behaviour with cognitive laws and mechanisms that are informed by neuroscience, but that cognitive laws/mechanisms must be replaced by neuroscientific laws/mechanisms. In the final section, we suggest that neuroscientific laws/mechanisms cannot replace cognitive laws and mechanisms (or psychological laws). Neuroscientific mechanisms fail to be reductions of cognitive mechanisms because they fail to be a "more comprehensive theory [that] contains [the] explanatory and predictive resources that parallel those of the reduced theory" (Bickle, 1998, p. 28). 


\section{A.4. An extended mind argument against reduction}

One of the standard defences against reductionism has been multiple realisability. However, for the reasons outlined in the preceding section, the truth of multiple realisability seems doubtful and, therefore, a defence against reductionism based on multiple realisability seems untenable. Instead, we propose an extended mind argument against reduction, which holds that the cognitive sciences cannot reduce to neuroscience because to generate models of intelligent behaviour we need to know about more than just what is happening in the brain. Neural explanations cannot replace cognitive explanations because they fail to parallel the predictive power, and richness, of cognitive explanations. In the following section, we will present this argument with the aim of demonstrating that the neural-centric view fails because it ignores the fundamental role that the organism's body and environment play in explaining intelligent behaviour; it is often the case that the (cognitive) mechanisms underlying intelligent behaviour reside not solely in the brain, but in the rich interaction between brains, bodies and environments.

This position lies in stark contrast to that of Bickle (2003), who puts forward a defence of what he calls "ruthless reductionism": the reduction of cognitive psychology not to cognitive neuroscience but rather to molecular and cellular neuroscience. Bickle's favoured example of such an approach-his "parade case for current reductionistic neuroscience" (p. 114) - is the reduction of long-term memory consolidation to molecular mechanisms of long-term potentiation (LTP), and he does not spare any details in his thorough treatment of the molecular mechanisms responsible for LTP. We commend this detailed molecular neuroscientific investigation just as we have commended connectionism and functional neuroimaging. Cellular and molecular neuroscience have an important part to play if they give us a more fine-grained account of those functional properties of neurons and neural networks that are causally relevant for the production of intelligent behaviour. Molecular neuroscience (especially studies of LTP) can, for example, provide us with information about changes in synaptic efficacy (connection weights) and their microfunctional consequences. Where we part company with Bickle, however, is in his conclusion that a detailed 


\section{A. Cognitive science does not reduce to neuroscience}

exposition of neural mechanisms naturally leads to reductionism. An examination of the extended mind argument will show why this conclusion is flawed.

The extended mind argument, as put forward by Clark and Chalmers (1998), simply stated, is that cognition is more than what happens in the head. On this view, those parts of the external environment on which certain aspects of cognition rely may be properly counted as part of the cognitive system producing intelligent behaviour. At the heart of the argument is the parity principle. In explaining the parity principle, Clark and Chalmers (1998) ask the reader to consider two situations: the first involves a subject performing some task entirely "in the head" through the mental manipulation of some internal symbol or representation; the second involves the same task being completed but this time with the previously internal symbols realised as some external symbol, representation, or artifact. They argue that if the first case of mental manipulation is to be considered cognitive then the second case, where physical manipulation of a physical object fills the same role as mental manipulation, should also be considered cognitive. It is important to note, however, that the extended mind argument that we endorse does not require a strong functional identity between extended and purely internal cognitive processes. Nor is it necessary that it must be possible for extended cognitive processes to also be done "in the head". Cognitive processes that extend into the body and environment do so because, often, these types of cognitive processes cannot efficiently, or easily, be performed in the head. In some cases, they cannot be performed in the head at all and, therefore, cognitive extension allows embodied organisms to acquire cognitive functions that might not otherwise be available to their (imagined) disembodied counterparts. In this way, certain cognitive processes rely as much on the body and the ability to manipulate the environment as they do on the brain. Remove any part and the mechanism collapses.

Much of the empirical research into the extended mind has drawn a distinction between what Kirsh and Maglio (1994) call "epistemic actions" and "pragmatic actions". Pragmatic actions are actions aimed at achieving some particular goal, such as walking towards an 
object that is desired. Epistemic actions, on the other hand, are physical actions that aid mental computation. This might be achieved by reducing memory requirements for, increasing the speed of, or increasing the reliability of, a particular computation. To illustrate the role of epistemic actions we turn to an example from the experimental literature.

Human working memory has a limited capacity (Cowan, 2001), which imposes constraints on any computational process that includes working memory. A computational advantage is to be had then if a cognitive system does not utilise working memory to its full capacity, but instead offloads information onto the environment or, alternatively, simply does not represent information in working memory at all, but rather extracts information from the environment when necessary. Evidence from eye-tracking studies suggests that people routinely do leave information out in the world accessing it as it is needed rather than representing it all internally. These studies suggest that eye movements can act as epistemic actions, aiding computation. An account of the role of eye movements (fixations and saccades) has been developed by Ballard, Hayhoe, Pook, and Rao (1997) with their notion of deictic pointers.

One pragmatic reason for fixation with the fovea is to increase the spatial resolution of the object being looked at. Ballard et al. (1997), however, suggest that fixation plays a role beyond merely increasing spatial resolution; they suggest that it plays a direct role in information processing. In a number of experiments using a block-copying paradigm, Ballard et al. (1997) asked subjects to duplicate a particular spatial arrangement of coloured blocks while their eye and hand movements were tracked. The results of these experiments showed that subjects made far more eye movements than would be expected if they were using working memory to its maximum capacity. Often, subjects would perform a saccade from the model to the block resource area, select a block, perform a saccade back to the model, and then to the assembly area before dropping the block in the correct location. Ballard et al. (1997) suggest that the saccade back to the model after the block has already been selected suggests that this saccade serves the purpose of retrieving the block's spatial location from the model, with the block's colour already retrieved (and pos- 


\section{A. Cognitive science does not reduce to neuroscience}

sibly now discarded from working memory) in the first fixation of the model. They suggest that this minimal memory strategy is preferred because of the high cost of maintaining items in working memory. Saccades, however, also carry a cost in terms of time, with saccades and on-line environmental retrieval being slower than internal retrieval from working memory.

The cost of on-line retrieval can be increased by increasing the spatial separation between the model block pattern and the block resource area, thereby introducing the need for head movements. In this case, the two competing costs (of body movements for on-line retrieval and working memory usage) are weighed against each other when selecting the strategy for performing the block-copying task. If the increase in temporal or metabolic costs is too great, then eye movements dramatically decrease and subjects utilise more working memory for this task, employing a maximal memory strategy (Ballard et al., 1997).

Ballard et al. (1997) suggest that these two strategies amount to two distinct computational approaches to solving the single problem of block copying. In the minimal memory strategy, eye movements serve as deictic pointers. A deictic pointer, like pointers in computer programming terminology, is a variable that stores the location of a piece of stored data. In this way, they differ from regular variables that store the data itself. In the blockcopying example, the piece of stored data might be the block's colour or spatial location. In the minimal memory strategy, this data is stored out in the environment; in the maximal memory strategy, this data is stored directly in working memory. Thus, eye movements in the minimal memory strategy serve to retrieve the data from the data store (the environment) based on the information stored in the (internal) pointer. In the maximal memory strategy, a functionally similar process retrieves the same data from the internal data store (working memory). In this way, each computational strategy can be functionally decomposed into parts (data store, central buffer) and operations (retrieval) yielding two, functionally similar, but not identical, cognitive/information-processing mechanisms. In one case, the parts of the mechanism are located solely inside the brain and the opera- 
tions are performed solely by the brain. In the other case, however, the parts are located in both the brain and the environment, and operations are performed by the brain and the body. According to the parity principle, this causal spread requires a spread of causal credit (Clark, 1997), and the result is causal equivalency between the neural and the extraneural.

Bickle (2008) has criticised the idea of causal equivalency between the neural and the extra-neural. He argues that the neural events have a "causal priority over all other causes of behaviour, beyond the fact... that brain events are the most proximal causes." (p. 472). In particular, Bickle (2008) takes issue with Clark and Chalmers (1998) when they state: "[I]f we retain internal structure but change the external features, behaviour may change completely. The external features here are just as causally relevant as typical internal features of the brain." (p. 9). According to Bickle (2008) there are two ways to interpret this statement. The first, he claims, is trivial and amounts to nothing more than the claim that the external world produces inputs for the brain with the brain maintaining causal priority over behaviour. The second, however, is "spooky" and "simply empirically implausible" (p. 472). This reading is "spooky" because, as Bickle (2008) quite rightly points out, changes in behaviour are not possible unless there is also a change in the particulars of neurotransmitter release at the neuromuscular junction. This, however, is not something that anybody would deny. Furthermore, accepting that changes in neurotransmitter release at the neuromuscular junction is required for changing behaviour amounts to nothing more than accepting that neural events are the most proximal causes of behaviour (save possibly for chemical changes in muscle fibres). However, for Bickle, neural events are the locus of intelligent behaviour over and above the fact that they are the most proximal causes of intelligent behaviour. Thus, it is clear that the "spooky" reading is not the one to be endorsed by extended mind theorists and Bickle's refutation of the "spooky" reading is accepted, although it does nothing to advance his argument. Therefore, it is clear that the issue is with the first reading. In particular, the issue is whether the environment should be merely characterised as input, as Bickle's first reading suggests, or whether it 


\section{A. Cognitive science does not reduce to neuroscience}

is something more. The studies by Ballard et al. (1997) might help to illustrate how the environment is more than just input.

The key finding from Ballard et al. (1997) is that changing the environment by moving the position of the model, the block resource area, and the assembly area has the effect of changing the computational algorithm or mechanism that is implemented: closer together and the larger mechanism includes neural operations that function as deictic pointers; further apart and the larger mechanism includes neural operations that "represent" objects and features of the outside world. Changes in the environment result in changes to the mechanism employed to complete the task by altering the division of labour between neural and extra-neural processes. Furthermore, if, when examining the environment-spanning mechanism, we conclude that those aspects of the physical environment that are causally relevant to behaviour are merely inputs then what is to stop one deciding that in the purely internal case the physical aspects of particular neural structures that are causally relevant to behaviour are also merely inputs: are the neural operations in V1 merely inputs to "higher" cortical areas or are they to be considered part of the mechanism? If the former is true, then where exactly in the causal chain does one draw the line between what is input and what is not? We agree with Chemero (2007) that psychology, and cognitive science, as we have presented it here, is the study of the brain-body-environment system. By "refus[ing] to grant the psychological any genuine causal explanatory role" (2003, p. 115), Bickle is suggesting that we ignore the way in which environmental differences change the mechanisms that are implemented and he thereby fails to appreciate the true causal role of the environment. In brushing over these differences, Bickle's reductionism results in a science of mind that focuses on only part of a much larger mechanism, and while it is true that the effects of environmental changes on behaviour are mediated/modulated by neural processes, it is certainly not the case that one can explain or predict behaviour based solely on a description of the neural parts and operations of this larger mechanism. Indeed, such a restriction fails to even recognise the extra-neural structures and processes as part of the mechanism! Bickle's commitment to a 
brain-only account of behaviour amounts to nothing more than an a priori assertion that those facts about the world that are important for understanding intelligent behaviour are those facts about the world that are studied by neuroscience.

\section{A.5. Conclusion}

For much of its history cognitive science has operated in a biological vacuum. This is a direct result of the view that the mind is the software of the brain. We have argued, however, that a clear-cut division between software and hardware does not exist. Rather, facts about how a system is implemented can (and does) have consequences for the functional (behavioural) properties of that system. Furthermore, we have tried to highlight the role that neuroscience has to play in theoretical progress in the field of cognitive psychology by examining how neuroscience, and neuroscientific reasoning about cognition, can focus attention on what might otherwise be neglected data. Neuroimaging, with its ability to uncover facts about brain function during certain tasks, informs cognitive psychology by enabling the generation of novel cognitive theories and behavioural predictions. However, although we urge a greater role for neuroscience within cognitive science, we suggest that reductionist tendencies should be resisted. If to understand intelligent behaviour we need to understand the dynamic interactions between brain, body and environment, as we have claimed, then brain reductionism with its singular focus only results in a decrease of explanatory power. Understanding the brain is vitally important to understanding intelligent behaviour, but it is only one part of the greater puzzle. Along with the downward trend of reductionism, there needs to be an outward expansion into the body and environment of biological organisms. It is only through this interdisciplinary endeavour that it will be possible to develop a better understanding of how intelligent behaviour is produced. 
A. Cognitive science does not reduce to neuroscience

\section{A.6. References}

Ballard, D. H., Hayhoe, M. M., Pook, P. K., \& Rao, R. P. (1997). Deictic codes for the embodiment of cognition. Behavioral and Brain Sciences, 20, 723-742.

Bickle, J. (1998). Psychoneural reduction: the new wave. Cambridge MA: MIT Press.

Bickle, J. (2003). Philosophy and neuroscience: a ruthlessly reductive account. Boston: Kluwer Academic Publishers.

Bickle, J. (2008). The molecules of social recognition memory: implications for social cognition, extended mind, and neuroethics. Consciousness and Cognition, 17, 468-474.

Buccino, G., Binkofski, F., Fink, G. R., Fadiga, L., Fogassi, L., Gallese, V., ... Freund, H. J. (2001). Action observation activates premotor and parietal areas in a somatotopic manner: an fMRI study. European Journal of Neuroscience, 13(2), 400-404.

Calvo-Merino, B., Glaser, D. E., Grézes, J., Passingham, R. E., \& Haggard, P. (2005). Action observation and acquired motor skills: an fMRI study with expert dancers. Cerebral Cortex, 15(8), 1243-1249.

Chemero, A. (2007). Asking what's inside the head: Neurophilosophy meets the extended mind. Minds and Machines, 17, 345-351.

Clark, A. (1989). Microcognition: philosophy, cognitive science, and parallel distributed processing. Cambridge MA: MIT Press.

Clark, A. (1997). Being there: putting brain, body, and world together again. Cambridge MA: MIT Press.

Clark, A., \& Chalmers, D. (1998). The extended mind. Analysis, 58, 7-19.

Coltheart, M. (2004). Brain imaging, connectionism, and cognitive neuropsychology. Cognitive Neuropsychology, 21, 21-25.

Cowan, N. (2001). The magical number 4 in short-term memory: a reconsideration of mental storage capacity. Behavioral and Brain Sciences, 24, 87-114.

Dobzhansky, T. (1973). Nothing in biology makes sense except in the light of evolution. The American Biology Teacher, 35, 125-129. 
Flach, R., Knoblich, G., \& Prinz, W. (2003). Off-line authorship effects in action perception. Brain and Cognition, 53(3), 503-513.

Gibson, J. J. (1986). The ecological approach to visual perception. New York: Psychology Press.

James, W. (1905). The principles of psychology. New York: Henry Holt and Company.

Kirsh, D., \& Maglio, P. (1994). On distinguishing epistemic from pragmatic action. Cognitive Science, 18, 513-549.

Lakatos, I. (1970). Falsification and the methodology of scientific research programmes. In I. Lakatos \& A. Musgrave (Eds.), Criticism and the growth of knowledge: volume 4: proceedings of the international colloquium in the philosophy of science, london, 1965 (pp. 91-196). Cambridge: Cambridge University Press.

McClelland, J. L. (1985). Putting knowledge in its place: a scheme for programming parallel processing structures on the fly. Cognitive Science, 9, 113-146.

Mozer, M. C. (1983). Letter migration in word perception. Journal of Experimental Psychology: Human Perception and Performance, 9, 531-546.

Newell, A., \& Simon, H. A. (1997). Computer science as empirical inquiry: symbols and search. In J. Haugeland (Ed.), Mind design II: philosophy, psychology, artificial intelligence (pp. 81-110). Cambridge MA: MIT Press.

Rizzolatti, G. (2005). The mirror neuron system and its function in humans. Anatomy and Embryology, 210, 419-421.

Shapiro, L. A. (2000). Multiple realizations. The Journal of Philosophy, 97, 635-654.

Stevens, J., Fonlupt, P., Shiffrar, M., \& Decety, J. (2000). New aspects of motion perception: selective neural encoding of apparent human movements. NeuroReport, 11(1), $109-115$.

Thagard, P. (1986). Parallel computation and the mind-body problem. Cognitive Science, 10, 301-318.

Wilson, M., \& Knoblich, G. (2005). The case for motor involvement in perceiving conspecifics. Psychological Bulletin, 131(3), 460-473. 